\title{
CMC hypersurfaces condensing to geodesic segments and rays in Riemannian manifolds
}

\author{
Adrian Butscher AND RAFE MAZZEO
}

\begin{abstract}
We construct examples of compact and one-ended constant mean curvature surfaces with large mean curvature in Riemannian manifolds with axial symmetry by gluing together small spheres positioned end-to-end along a geodesic. Such surfaces cannot exist in Euclidean space, but we show that the gradient of the ambient scalar curvature acts as a 'friction term' which permits the usual analytic gluing construction to be carried out.
\end{abstract}

Mathematics Subject Classification (2010): 53A10 (primary); 35J93, 35B25 (secondary).

\section{Introduction}

Background. The study of constant mean curvature (CMC) surfaces in $\mathbb{R}^{3}$, or in more general three-dimensional Riemannian manifolds, is a well established field of Riemannian geometry and there is a vast literature concerning the construction and properties of such surfaces. One particular method for constructing CMC surfaces is by analytic gluing techniques. This goes back to the work of Kapouleas [6,7] and has been further developed by many others, including the first author with Pacard [1,2] and the second author with Pacard and also with Pollack [10,12]. (See [13] and [16] for surveys about the current state of this approach.) The general idea in this approach is to take connected sums of simple surfaces, e.g. the sphere, cylinder or Delaunay surfaces, joined by approximately catenoidal necks, to produce 'approximate CMC' surfaces and then perturbing these by PDE arguments to obtain surfaces with exactly constant mean curvature.

The simplest examples of complete noncompact embedded CMC surfaces in $\mathbb{R}^{3}$ are the Delaunay surfaces; these are the unique rotationally symmetric CMC surfaces and each has a discrete translational symmetry as well. This family interpolates between the cylinder and an infinite string of mutually tangent spheres arranged along an axis; elements of this family are distinguished by a 'necksize' parameter which measures the length of the shortest closed geodesic.

R. M. supported by the NSF Grant DMS-0805529.

Received January 10, 2009; accepted in revised form March 22, 2011. 
The structure of more general complete, Alexandrov-embedded CMC surfaces with finite topology in $\mathbb{R}^{3}$ is fairly well understood. Meeks [14] proved that each end of such a surface is cylindrically bounded; Korevaar, Kusner and Solomon [8] subsequently showed that each end converges exponentially to the end of some Delaunay surface. Hence to any such surface we may assign a set of parameters describing the asymptotic Delaunay surface corresponding to each end. There are limitations on the asymptotic parameters which can be achieved by complete CMC surfaces, as well as a moduli space theory which describes the infinitesimal and local variations of these asymptotic parameters as one varies the $\mathrm{CMC}$ surface, see [3-5] and [9].

An alternative description of these asymptotic parameters is provided by the flux integral, discovered by Kusner, which is a vector obtained by integrating a locally defined quantity over any closed loop in a surface. When the surface is CMC, this only depends on the homology class of the loop, and in particular, the flux of a simple positively oriented loop around each end determines the direction of the axis and necksize, but not the 'phase' of the translation, of the asymptotic Delaunay model for that end. The homological invariance also shows that the sum of these fluxes over all ends must vanish; this is a global balancing condition for the CMC surface, which is the simplest of the limitations referred to above. For example, an immediate consequence is that there exists no complete Alexandrov-embedded CMC surface in $\mathbb{R}^{3}$ with only one end. However, these fluxes play an even more central role in the gluing theory: one of the key features of the approximate CMC configurations which allows them to be perturbed to be exactly $\mathrm{CMC}$ is that the total flux over each gluing region (i.e. the necks in the connected sum) is nearly zero. In fact, as we explain more carefully below, there are other local balancing conditions, and also a certain global 'flexibility', stated in terms of fluxes, which are crucial for the analytic method to be carried out.

Our goal in this paper is to construct new examples of CMC surfaces in more general three-manifolds which exhibit new types of behaviour not possible in Euclidean space. These are once again perturbations of collections of small spheres joined together by even smaller catenoidal necks, all arranged along a curve $\gamma$. As one expects with these gluing constructions, the sizes of the catenoidal necks are quite small compared to the radii of the spheres. However, quite strikingly, these neck-sizes must vary along this chain of spheres. This variation is due to a new contribution in the flux integral from the scalar curvature $S$ of the ambient manifold. Indeed, in this curved setting, the flux over a curve which is homologically trivial need not vanish, but can be expressed in terms of a surface integral involving $\nabla S$. This is completely analogous to the generalized Pohozaev identity discovered by Schoen which arises in his construction of metrics of constant positive scalar curvature [19]. In our setting, this shows that the difference between successive neck sizes in the initial approximate CMC configuration must involve the gradient of the scalar curvature along the axis connecting these two necks. In other words, $\nabla S$ acts as a 'friction' which forces a change in the size of the connections between adjacent spheres along $\gamma$. Our central result states that if this effect is managed correctly, one can find exact CMC surfaces where the string of spheres eventually 
'caps off' at one or both ends, and thus we obtain CMC surfaces arranged along an interval or ray.

To explain this all more carefully, and also to set it into final context, we discuss a newer theme in this subject. In Euclidean space, dilation by the scalar $\lambda$ transforms a CMC surface with mean curvature $H$ into one with mean curvature $\lambda^{-1} H$; hence the precise value of the mean curvature is irrelevant (so long as it is nonzero). By contrast, in a curved 3-manifold there is a marked contrast between the study of CMC surfaces with 'small' mean curvatures, which tend to be very rigid objects, and those with very large mean curvature. We focus here on the latter. The general expectation is that a sequence of CMC surfaces with mean curvature tending to infinity should 'condense' onto a curve, or perhaps some slightly more complicated one-dimensional set. For example, if $\Sigma$ is a fixed (complete, Alexandrov-embedded) surface with mean curvature $H \equiv 2$ in $\mathbb{R}^{3}$, and if $\epsilon_{j} \rightarrow 0$, then $\epsilon_{j} \Sigma$ has $H=\epsilon_{j}^{-1}$, and by the structure theory discussed above, this sequence condenses onto a union of half-lines emanating from the origin. This seems to be a general phenomenon: Rosenberg [18] has shown that if $\Sigma$ is a closed CMC surface in an arbitrary compact 3-manifold $M$, with mean curvature $H \gg 0$, then $M \backslash \Sigma$ has two components, and the inradius at any point in one of these components is bounded above by $C / H$. In other words, $\Sigma$ is contained in - and perhaps looks like - a tube around some (presumably 1-dimensional) set $\gamma$. This leads to the following central question:

Question: What are the possible condensation sets $\gamma$ in a 3-manifold $M$ for sequences of CMC surfaces $\Sigma_{j}$ with mean curvatures $H_{j} \nearrow \infty$ ?

This might be regarded as some sort of CMC analogue of the problem, famously studied in depth by Colding and Minicozzi, about the behaviour of sequences of minimal surfaces without area bounds.

One indication about the possible nature of the condensation set is provided by a formal calculation, which shows that if $\Sigma_{j}$ is a sequence of CMC surfaces with $H_{j} \rightarrow \infty$ condensing to a smooth curve $\gamma$, and if we assume that the pointwise norm of the second fundamental form on $\Sigma_{j}$ is everywhere comparable to $H_{j}$, then $\gamma$ is a geodesic. This and the previous example of dilated surfaces in $\mathbb{R}^{3}$ leads to the conjecture that the condensation set must be a network of geodesic arcs and rays. In fact, one might even conjecture that each edge of this geodesic network inherits a 'weight' encoding the Delaunay parameters of the CMC tubular piece converging to that edge.

As a preliminary step toward understanding this condensation phenomenon, the second author and Pacard [11] proved that if $\gamma$ is any closed geodesic in $M$ which is non-degenerate (in the sense that its Jacobi operator is invertible), then geodesic tubes of sufficiently small radius about $\gamma$, at least for most radii, can be perturbed to CMC surfaces with large $H$.

The present paper contains one further step toward understanding this condensation phenomenon by showing that at least for certain very special ambient geometries, there exist sequences of CMC surfaces which condense to a geodesic 
ray or a finite geodesic interval. However, one of the central points of our construction also points in a different direction and indicates that the geodesic nature of the limiting set $\gamma$ is not really the crucial feature, but rather, that the gradient of the ambient scalar curvature plays an equally important role in the geometry of this condensation set.

We have already noted that the scalar curvature $S$ contributes to the flux integral. However, this function has appeared elsewhere in this theory too. Indeed, the first result about CMC surfaces with large $H$, by Ye [20] in the early 1990's, states that if $p$ is a non-degenerate critical point of the scalar curvature function $S$ (in a manifold $M$ of any dimension), then the geodesic spheres $B_{p}(r)$ with small radius may be perturbed to $\mathrm{CMC}$ surfaces $H \sim 1 / r$. He also showed a partial converse: a sequence of CMC spheres with bounded eccentricity and with $H \rightarrow \infty$ converge to a point $p$ where $\nabla S(p)=0$. By contrast, the scalar curvature of $M$ plays no role in the location of the geodesic which is the condensation set in [11]. In light of the construction here, this is almost surely because the CMC surfaces constructed there are nearly cylindrical.

The balancing formula. We finally provide come to a more quantitative description of our results. Let $\Sigma$ be a surface with constant mean curvature $H$ in $\left(M^{3}, g\right)$. Suppose that $\mathcal{U}$ is an open set in $\Sigma$, and let $\mathcal{W}$ be another open set in $M$ with piecewise smooth boundary such that $\partial \mathcal{W} \cap \Sigma=\overline{\mathcal{U}}$. (The other portion of $\partial \mathcal{W}$ is denoted $Q$.) If $X$ is a Killing field on $M$, then the first variation formula for the area of $\mathcal{U}$ with the volume of $\mathcal{W}$ fixed relative to the one-parameter family of diffeomorphisms for $X$ gives that

$$
\int_{\partial \mathcal{U}} g(v, X)-H \int_{Q} g(N, X)=0
$$

here $v$ is the unit normal vector field of $\partial \mathcal{U}$ in $\Sigma$ and $N$ is the unit normal vector field of $Q$ in $M$. More generally, the flux itself is defined as

$$
\int_{\gamma} g(v, V)-H \int_{Q} g(N, V)
$$

where $\gamma$ is a curve in $\Sigma, Q$ is any surface in $M$ with $\partial Q=\gamma$, and $V$ is any vector field on $M$. This integral is always independent of the choice of $Q$, and if $\Sigma$ has CMC and $V$ is a Killing vector field, then (1.1) states that the flux depends only on the homology class of $\gamma$ in $\Sigma$. These formulæ first appeared in [8] (for surfaces in $\mathbb{R}^{3}$ ).

Let us explain how these flux integrals help to determine when an approximately CMC surface can be perturbed to be exactly CMC. Suppose first that $M=$ $\mathbb{R}^{3}$ and let $\Sigma$ consist of a collection of spheres of radius $r$ (hence mean curvature $n / r$ ) connected to each other by small catenoidal necks. Let $\mathcal{U}$ be one of these spheres with small spherical caps removed where the necks are attached, $Q$ the union of disks capping these boundaries and $\mathcal{W}$ the slightly truncated ball enclosed 
by $\mathcal{U} \cup Q$. Then (1.1) becomes

$$
\int_{\partial \mathcal{U}} g(\nu, V)-\frac{2}{r} \int_{Q} g(N, V)=\sum_{\text {all necks }} r \varepsilon_{i} V_{i}+\mathcal{O}\left(r \varepsilon^{2}\right),
$$

where $V_{i}$ is the unit vector pointing from the center of sphere in question to the $i^{\text {th }}$ neck, $r \varepsilon_{i}$ is the width of this neck and $\varepsilon:=\max _{i}\left\{\varepsilon_{i}\right\}$. If $\Sigma$ were exactly CMC, the left hand side would necessarily vanish. If $\Sigma$ is not exactly CMC, then to find a nearby CMC surface, the leading term on the right in (1.3) must vanish for each spherical region $\mathcal{U}$ in $\Sigma$. If this condition is satisfied, the approximate CMC surface $\Sigma$ is called balanced. Note that there is no balanced approximately CMC surface where some sphere has only one spherical neighbour.

Now let $\Sigma$ be an approximately surface formed by attaching together some large collection of geodesic spheres of very small radius $r$ in a more general $\left(M^{3}, g\right)$. Although there are usually no ambient Killing fields, we can still use the approximate Killing fields corresponding to translations and rotations in Riemann normal coordinates based at the center of any one of these spheres. Formula (1.1) now becomes

$$
\int_{\partial \mathcal{U}} g(v, V)-H \int_{Q} g(N, V)=\sum_{\text {all necks }} r \varepsilon_{i} V_{i}-C r^{4} \nabla S(p)+\mathcal{O}\left(r \varepsilon^{2}\right)+\mathcal{O}\left(r^{6}\right)
$$

where $\nabla S$ is the gradient of the scalar curvature of $M$ and $C$ is an explicit dimensional constant. Note that when applied to a sphere with no neighbours, i.e. all $\varepsilon_{i}=0$, this gives a condition which appears in Ye's work that the right hand side of (1.4) must vanish like $r^{6}$.

The main point in this paper is to exploit the contribution of $\nabla S$ in (1.4). Spheres of radius $r$ are joined by necks of width $r \varepsilon$, where by this same formula it is natural to assume that $\varepsilon=\mathcal{O}\left(r^{3}\right)$; these configurations are arranged in such a way that the leading term on the right in (1.4) vanishes. The perturbation argument used to produce a nearby CMC surface is not so different from the ones appearing elsewhere in the literature, so the novelty here is the new method of constructing balanced approximate CMC configurations.

Description of the surfaces. Our goal here is a 'demonstration of concept' rather than the most general possible construction based on the considerations above. We shall produce two specific types of condensing sequences of CMC surfaces which exhibit markedly different properties than those which had been observed previously. As will be evident, the technicalities are formidable, so in order to minimize these and illuminate the main ideas, we shall focus on some very special examples inside three-manifolds $M$ of a very special type. This provides a very good illustration about what should happen in more general situations, although we do not make any claims about what actually happens in these more general situations here. More specifically, we assume that $(M, g)$ contains a geodesic (ray or interval) $\gamma$, in some neighbourhood of which the metric has an axial symmetry. In other words, 
suppose that $\gamma$ is parametrized by arc length $t$ and that in some neighbourhood of $\gamma$, in Fermi coordinates the metric is given by

$$
g=d t^{2}+A(t) \delta
$$

here $\delta$ is the Euclidean metric on $D$ and $A(t)$ is a smooth, strictly positive function of the arclength $t$ along $\gamma$. The initial configurations here are a union of geodesic spheres arranged along $\gamma$ and connected by small catenoidal necks. Semi-infinite configurations are obtained by attaching to these one half of a Delaunay surface with small neck size. In either case, each component has at most two neighbours.

The scalar curvature of $g$ is $S:=A^{-2}\left(-2 A \ddot{A}+\frac{1}{2} \dot{A}^{2}\right)$. We shall place different conditions on the warping function $A$ depending on whether we are trying to obtain finite-length or one-ended CMC surfaces.

1. To construct surfaces condensing to a geodesic interval, assume that $A$ is an even function of $t$, and that $t=0$ is a non-degenerate local maximum of $S$.

2. To construct one-ended surfaces condensing to a geodesic ray, assume that when $t>0$, the scalar curvature is negative, monotonically increasing, and satisfies $|S(t)| \leq C \mathrm{e}^{-\alpha t}$ for some $\alpha>0$. (An example is $A(t):=1+\mathrm{e}^{-t}$.)

Note that in either case, $\nabla S$ points along $\gamma$.

As already noted, these assumptions significantly reduce the complexity of the perturbation argument. The great defect in considering only this special case is that $\gamma$ is not only a geodesic for $g$ but also an integral curve for $\nabla S$. It is unclear which of these features is the crucial one, and in fact we expect that in more general ambient geometries, the condensation curves will have some sort of mixed characterization, i.e. be determined by a differential equation which is some perturbation of the geodesic equation by a term involving $\nabla S$.

Our main result can now be expressed as follows.

Theorem. Let $M^{3}$ be a Riemannian manifold with the special features described above.

- Let $I=\gamma([-L, L])$ where $\gamma$ is the central geodesic and $L<\infty$. Then there exists an $r_{0}>0$ so that for every $0<r<r_{0}$, there is a CMC surface $\Sigma_{r}^{F}$ which is a small perturbation of a surface constructed by gluing together length $(I) / r$ spheres of radius $r$ with centers lying on $\gamma$.

- Let $I=\gamma([0, \infty))$. Then there exists an $r_{0}>0$ so that for every $0<r<r_{0}$, there is a CMC surface $\Sigma_{r}^{O E}$ which is a small perturbation of a surface constructed by gluing together a number $O(1 / r)$ spheres of radius $r$ with centers lying on $\gamma$, together with an end of a Delaunay surface whose axis lies along $\gamma$.

The rescalings of $\Sigma_{r}^{F}$ and $\Sigma_{r}^{O E}$ by the factor $1 / r$ converge as $r \rightarrow 0$ to an infinite or semi-infinite string of spheres of radius 1 with centers arranged along a segment or ray in $\mathbb{R}^{3}$. More details about this convergence will be stated in the course of the proof. 
As we have explained, the proof follows the strategy of previous gluing theorems for CMC surfaces. After constructing the initial balanced approximately CMC surface $\tilde{\Sigma}$ (by gluing together small geodesic spheres and catenoidal necks) we seek to perturb this approximate solution into an exact solution using small normal deformations parametrized by functions on $\tilde{\Sigma}$. This transforms the problem into a nonlinear elliptic PDE on $\tilde{\Sigma}$. However, $\tilde{\Sigma}$ is close to CMC only when all parameters (spherical radii and necksizes) are very small, so we face the difficulty that we must solve this PDE only near this degenerate limit. There are now three steps. First we must invert the linearized mean curvature operator (i.e. the Jacobi operator) $\mathcal{L}$ with suitable bounds on the inverse. One now-standard way to do this, used already by Kapouleas [6,7], is by semi-localization. To solve $\mathcal{L} u=f$, first solve this problem on each building block and then combine these solutions appropriately (with a perturbation argument) to construct a solution on all of $\tilde{\Sigma}$. Kapouleas does this using the Dirichlet problem on each building block, considered as surfaces with boundary, e.g. the spheres with small disks excised. We use the slightly different (but equivalent) approach of studying the Jacobi operators on the limits of the building blocks, where the parameters have tended to zero. In either approach, the main technical difficulty is the existence of small eigenvalues for the Jacobi operators on each building block, and we must identify these eigenspaces, at least approximately, so as to be able to work orthogonally to them. The second step of the construction is to use the (generalized) inverse of the Jacobi operator in a fixedpoint argument to solve the non-linear PDE on the orthogonal complement of the approximate nullspace of the linearized PDE. Here this approximate nullspace is finite dimensional. This yields a solution whose mean curvature is constant modulo a term in this approximate nullspace. The final step shows that by repositioning the various building blocks relative to one other, one can kill the remaining approximate nullspace term. The balancing formula (1.1) plays an essential role here. The quantitative estimates throughout all steps of the proof involve keeping careful track of dependence on the neck sizes and radii, and this necessitates the introduction of various weighted function spaces. These steps constitute the approximate plan for the layout of this paper.

As a final remark, and to address a comment of the referee, let us note that since our strong symmetry assumptions reduce this problem to an ordinary differential equation, it may be possible (as the referee suggests) to use an ODE shooting method to prove our main results. Even this approach would be very subtle though since one wishes to find highly oscillatory solutions with a large prescribed number of slightly positive minima. There are indeed results of this type in dynamical systems. We do not follow this line of argument, both because it would not properly illuminate the role of how $\nabla S$ enters the balancing conditions, but also because it sheds no light on the more general non-symmetric situation.

ACKNOWLedgements. The authors wish to thank Frank Pacard for interesting discussions and encouragement during the course of this work. They also thank the referee for carefully reading this paper and for many constructive suggestions on how to improve the exposition. 


\section{Preliminary geometric calculations}

The approximate solutions constructed in Section 3 are assembled from small geodesic spheres centered on points of the geodesic $\gamma$ in $M$ connected to one another by small catenoidal necks. It is most convenient to use geodesic normal coordinates centered at points of $\gamma$. Since the ambient metric $g$ is a second order perturbation of the Euclidean metric in these coordinates, the first step in every estimate is to perform the computations for a Euclidean metric; the second step is to incorporate the perturbations coming from the metric into the estimates. In this section we derive various expansions of the mean curvature and other geometric quantities that will be used routinely in what follows.

\subsection{Geometry of surfaces in a geodesic normal coordinate chart}

If $p$ is any point in a Riemannian manifold $(M, g)$, then in terms of geodesic normal coordinates centered at $p$,

$$
g:=\stackrel{\circ}{g}+P:=\left(\delta_{i j}+P_{i j}(x)\right) \mathrm{d} x^{i} \otimes \mathrm{d} x^{j}
$$

where $g$ is the Euclidean metric and $P$ is the perturbation term. It is well known that

$$
P_{i j}(x)=\frac{1}{3} \sum_{l, m} R_{i l j m}(0) x^{l} x^{m}+\frac{1}{6} \sum_{l, m, n} R_{i l j m ; n}(0) x^{l} x^{m} x^{n}+\mathcal{O}\left(\|x\|^{4}\right) .
$$

where $R_{i j k l}:=\operatorname{Rm}\left(\frac{\partial}{\partial x^{i}}, \frac{\partial}{\partial x^{j}}, \frac{\partial}{\partial x^{k}}, \frac{\partial}{\partial x^{l}}\right)$ and $R_{i j k l ; m}:=\bar{\nabla}_{\frac{\partial}{\partial x^{m}}}, \operatorname{Rm}\left(\frac{\partial}{\partial x^{i}}, \frac{\partial}{\partial x^{j}}, \frac{\partial}{\partial x^{k}}, \frac{\partial}{\partial x^{l}}\right)$ are components of the ambient Riemann curvature tensor and its covariant derivative. (The ambient covariant derivative with respect to $g$ is here denoted $\bar{\nabla}$. Let us also denote the ambient covariant derivative with respect to the Euclidean metric of the coordinate chart by D.)

Suppose that $\Sigma$ is a surface in a geodesic normal coordinate chart of $M$. The following results provide expansions for various geometric quantities of $\Sigma$ in terms of $P$. Here and in the rest of the paper, let $h, \Gamma, \nabla, \Delta, N, B, H$ be the induced metric, Christoffel symbols, covariant derivative, Laplacian, unit normal vector, second fundamental form, and mean curvature of $\Sigma$ with respect to the metric $g$, and let $\stackrel{\circ}{h}, \stackrel{\circ}{\Gamma}, \stackrel{\circ}{\nabla}, \stackrel{\circ}{\Delta}, \stackrel{\circ}{N}, \stackrel{\circ}{B}, \stackrel{\circ}{H}$ be these same objects with respect to the Euclidean metric in the coordinate chart. Let $\left\{E_{1}, E_{2}\right\}$ be a local coordinate frame for $T \Sigma$ near $x \in \Sigma$. Define the linear operator $\Upsilon$ by $\Upsilon(X, Y, Z):=\frac{1}{2}(X \otimes Y \otimes Z+Y \otimes$ $X \otimes Z-Z \otimes X \otimes Y)$ and introduce the notation $X_{A}:=E_{j}$ when $A=j$ and $X_{A}=\stackrel{\circ}{N}$ when $A=0$. Now define

$$
\begin{aligned}
\mathcal{P}_{A B} & :=P\left(X_{A}, X_{B}\right) \\
\mathcal{P}_{A B C} & :=\mathrm{D} P \circ \Upsilon\left(X_{A}, X_{B}, X_{C}\right) .
\end{aligned}
$$

Straightforward geometric calculations then yield the following results. 
Lemma 2.1. The induced metric of $\Sigma$ and the associated Christoffel symbols are given by

$$
h_{i j}=\stackrel{\circ}{h}_{i j}+\mathcal{P}_{i j} \quad \text { and } \quad \Gamma_{i j k}=\stackrel{\circ}{\Gamma}_{i j k}+\mathcal{P}_{i j k}+\mathcal{P}_{0 k} \stackrel{\circ}{B}_{i j}
$$

The normal vector of $\Sigma$ satisfies

$$
N=\frac{\stackrel{\circ}{N}-h^{i j} \mathcal{P}_{0 j} E_{i}}{\left(1+\stackrel{\circ}{P}_{00}-\stackrel{h}{i j} \mathcal{P}_{0 i} \mathcal{P}_{0 j}\right)^{1 / 2}}
$$

The second fundamental form of $\Sigma$ satisfies

$$
B_{i j}=\left(1+\mathcal{P}_{00}-\stackrel{\circ}{h}^{i j} \mathcal{P}_{0 i} \mathcal{P}_{0 j}\right)^{1 / 2} \stackrel{\circ}{B}_{i j}+\frac{\mathcal{P}_{i j 0}-\stackrel{\circ}{h}^{k l} \mathcal{P}_{0 k} \mathcal{P}_{i j l}}{\left(1+\mathcal{P}_{00}-\stackrel{\circ}{h}^{i j} \mathcal{P}_{0 i} \mathcal{P}_{0 j}\right)^{1 / 2}}
$$

We will need to make many more comparisons between geometric objects defined on a surface $\Sigma$ computed with respect to the Euclidean metric and those same objects computed with respect to the metric $g=\delta+P$. We prove here the most important ones. Let $Y:=\sum_{i} x^{i} \frac{\partial}{\partial x^{i}}$ be the position vector field in the coordinate chart; we will also use $Y$ to denote this vector field restricted to $\Sigma$. In the estimates that follow, let $[h]_{\alpha}:=\sup _{x_{1}, x_{2}}\left|h\left(x_{1}\right)-h\left(x_{2}\right)\right| / \operatorname{dist}\left(x_{1}-x_{2}\right)^{\alpha}$ denote the Hölder seminorm of a function $h$ in the coordinate chart. We will also make the assumption that $\|Y\|\|\stackrel{\circ}{B}\|+\|Y\|^{1+\alpha}[\stackrel{\circ}{B}]_{\alpha} \leq C$ where $C$ is some constant depending only on the curvature tensor of $M$ in the coordinate chart. This will be justified later on.

Lemma 2.2. If the second fundamental form of $\Sigma$ satisfies $\|Y\|\|\stackrel{\circ}{B}\|+\|Y\|^{1+\alpha}[\stackrel{\circ}{B}]_{\alpha} \leq$ $C$ then the operators $\mathcal{L}:=\Delta+\|B\|^{2}$ and $\stackrel{\mathcal{L}}{:}:=\stackrel{\circ}{\Delta}+\|\stackrel{\circ}{B}\|^{2}$ satisfy

$$
\begin{aligned}
& |\mathcal{L}(u)-\stackrel{\circ}{\mathcal{L}}(u)|+\|Y\|^{\alpha}[\mathcal{L}(u)-\stackrel{\circ}{\mathcal{L}}(u)]_{\alpha} \\
& \quad \leq C\left(|u|+\|Y\|\|\stackrel{\circ}{\nabla} u\|+\|Y\|^{2}\left\|\stackrel{\circ}{\nabla}^{2} u\right\|+\|Y\|^{2+\alpha}\left[\stackrel{\circ}{\nabla}^{2} u\right]_{\alpha}\right)
\end{aligned}
$$

where $C$ is a constant depending only on the geometry of $M$ in the coordinate chart.

Proof. We use the expressions for $h, \Gamma, B$ in terms of $\stackrel{\circ}{h}, \stackrel{\circ}{\Gamma}, \stackrel{\circ}{B}$ from Lemma 2.1 . Without loss of generality, we can assume that the coordinates satisfy $\stackrel{\circ}{\Gamma}=0$. Thus we have

$$
\begin{aligned}
|\Delta u-\stackrel{\circ}{\Delta} u| & \leq\left|h^{i j}-\stackrel{\circ}{h}^{i j}\right|\left|u_{, i j}\right|+\left|h^{s t} \Gamma_{i j t} \| u_{, s}\right| \\
& \leq C\left(\|Y\|^{2}\left\|\stackrel{\circ}{\nabla}^{2} u\right\|+\|Y\|(1+\|Y\|\|\stackrel{\circ}{B}\|)\|\stackrel{\circ}{\nabla} u\|\right)
\end{aligned}
$$

since $h^{i j}-\stackrel{\circ}{h}^{i j}=\mathcal{A}_{2}(Y)$ and $\Gamma_{i j t}=\left[\mathcal{A}_{1}(Y)\right]_{i j t}+\left[\mathcal{A}_{2}(Y)\right]_{t} \stackrel{\circ}{B}_{i j}$ where $\mathcal{A}_{k}(z)$ is a degree- $k$ analytic function. Next, re-using the above results together with the fact 
that the derivative $\mathcal{A}_{k}^{\prime}(z)=\mathcal{A}_{k-1}(z)$ and that $[Y]_{\alpha} \leq\|Y\|^{1-\alpha}$ we obtain

$$
\begin{aligned}
& {[\Delta u-\stackrel{\triangleright}{\Delta} u]_{\alpha}} \\
& \leq\left[h^{i j}-\stackrel{\circ}{h}^{i j}\right]_{\alpha}\left|u_{, i j}\right|+\left[h^{s t} \Gamma_{i j t}\right]_{\alpha}\left|u_{, s}\right|+\left|h^{i j}-\stackrel{\circ}{h}^{i j}\right|\left[u_{, i j}\right]_{\alpha}+\left|h^{s t} \Gamma_{i j t}\right|\left[u_{, s}\right]_{\alpha} \\
& \leq\left|\mathcal{A}_{2}^{\prime}(Y)\right|[Y]_{\alpha}\left\|\stackrel{\circ}{\nabla}^{2} u\right\| \\
& +\left(\left|\mathcal{A}_{1}^{\prime}(Y)\right|[Y]_{\alpha}+\left|\mathcal{A}_{2}^{\prime}(Y)\right|[Y]_{\alpha}\|\stackrel{\circ}{B}\|+\left|\mathcal{A}_{2}(Y)\right|[\stackrel{\circ}{B}]_{\alpha}\right)\|\stackrel{\circ}{\nabla} u\| \\
& +\|Y\|^{2}\left[\stackrel{\circ}{\nabla}^{2} u\right]_{\alpha}+\|Y\|\left[\nabla^{\nabla} u\right]_{\alpha} \\
& \leq\|Y\|^{2-\alpha}\left\|\stackrel{\circ}{\nabla}^{2} u\right\|+\left(1+\|Y\|\|\stackrel{\circ}{B}\|+\|Y\|^{1+\alpha}[\stackrel{\circ}{B}]_{\alpha}\right)\|Y\|^{1-\alpha}\|\stackrel{\circ}{\nabla} u\| \\
& +\|Y\|^{2}\left[\stackrel{\circ}{ }^{2} u\right]_{\alpha}+\|Y\|[\stackrel{\circ}{\nabla} u]_{\alpha} \text {. }
\end{aligned}
$$

Finally, we use the form $B=\left(1+\mathcal{A}_{2}(Y)\right) \stackrel{\circ}{B}+\mathcal{A}_{1}(Y)$ to derive

$$
\begin{aligned}
\left|\|B\|^{2} u-\|\stackrel{\circ}{B}\|^{2} u\right| & \leq C \max \{\|B\|,\|\stackrel{\circ}{B}\|\} \cdot\|B-\stackrel{\circ}{B}\| \cdot|u| \\
& \leq C\|\stackrel{\circ}{B}\|\left(\left\|\mathcal{A}_{2}(Y)\right\|\|\stackrel{\circ}{B}\|+\left\|\mathcal{A}_{1}(Y)\right\|\right)|u| \\
& \leq C\|Y\|\|\stackrel{\circ}{B}\||u|
\end{aligned}
$$

and

$$
\begin{aligned}
& {\left[\|B\|^{2} u-\|\stackrel{\circ}{B}\|^{2} u\right]_{\alpha}} \\
& \leq\left[\|B\|^{2}-\|\stackrel{\circ}{B}\|^{2}\right]_{\alpha}|u|+\left|\|B\|^{2}-\|\stackrel{\circ}{B}\|^{2}\right|[u]_{\alpha} \\
& \leq C\left(\max \left\{[B]_{\alpha},[\stackrel{\circ}{B}]_{\alpha}\right\} \cdot\|B-\stackrel{\circ}{B}\|+\max \{\|B\|,\|\stackrel{\circ}{B}\|\} \cdot[B-\stackrel{\circ}{B}]_{\alpha}\right)|u| \\
& \quad+C\|Y\|\|\stackrel{\circ}{B}\|[u]_{\alpha} \\
& \quad C \quad\left(\|Y\|(1+\|Y\|\|\stackrel{\circ}{B}\|)[\stackrel{\circ}{B}]_{\alpha}+C\|\stackrel{\circ}{B}\|\|Y\|^{1-\alpha}\left(1+\|Y\|\|\stackrel{\circ}{B}\|+\|Y\|^{1+\alpha}[\stackrel{\circ}{B}]_{\alpha}\right)\right. \\
& \left.\quad+\|Y\|\|\stackrel{\circ}{B}\|[u]_{\alpha}\right) .
\end{aligned}
$$

The desired estimates follow by combining all the above results.

Lemma 2.3. If the second fundamental form of $\Sigma$ satisfies $\|Y\|\|\stackrel{\circ}{B}\|+\|Y\|^{1+\alpha}[\stackrel{\circ}{B}]_{\alpha} \leq$ $C$ then the mean curvature of $\Sigma$ satisfies

$$
\left|H-\stackrel{\circ}{H}-\mathcal{H}^{(00)}(Y, \stackrel{\circ}{B})\right|+\|Y\|^{\alpha}\left[H-\stackrel{\circ}{H}-\mathcal{H}^{(00)}(Y, \stackrel{\circ}{B})\right]_{\alpha} \leq C\|Y\|^{3}
$$

where

$$
\begin{aligned}
\mathcal{H}^{(00)}(Y, \stackrel{\circ}{B}):= & \frac{1}{6}\left(\operatorname{Rm}(\stackrel{\circ}{N}, Y, \stackrel{\circ}{N}, Y)+\frac{1}{2} \bar{\nabla}_{Y} \operatorname{Rm}(\stackrel{\circ}{N}, Y, \stackrel{\circ}{N}, Y)\right) \stackrel{\circ}{H} \\
& -\frac{1}{3}\left(\operatorname{Rm}\left(E_{i}, Y, E_{j}, Y\right)+\frac{1}{2} \bar{\nabla}_{Y} \operatorname{Rm}\left(E_{i}, Y, E_{j}, Y\right)\right) \stackrel{\circ}{B}^{i j} \\
& -\frac{2}{3} \operatorname{Ric}(Y, \stackrel{\circ}{N})-\frac{1}{2} \bar{\nabla}_{Y} \operatorname{Ric}(Y, \stackrel{\circ}{N})+\frac{1}{12} \bar{\nabla}_{\stackrel{\circ}{N}} \operatorname{Ric}(Y, Y) \\
& -\frac{1}{6} \bar{\nabla}_{\stackrel{\circ}{N}} \operatorname{Rm}(\stackrel{\circ}{N}, Y, \stackrel{\circ}{N}, Y)
\end{aligned}
$$


and $\mathrm{Rm}_{p}$ and $\mathrm{Ric}_{p}$ are the Riemann curvature tensor and Ricci tensor $M$ of the ambient manifold at the centre of the coordinate chart and $C$ is a constant depending only on the geometry of $M$ in the coordinate chart.

Proof. The starting point is once again Lemma 2.1 from which we deduce that the induced metric of $\Sigma$ satisfies $h^{i j}=h^{i j}-\grave{h}^{i s} \stackrel{\circ}{j t}^{j t} \mathcal{P}_{s t}+\mathcal{A}_{4}(Y)$ where we use the same notation for $\mathcal{A}_{k}$ as in the previous lemma. Next, we can expand

$$
B_{i j}=\left(1+\frac{1}{2} \mathcal{P}_{00}\right) \stackrel{\circ}{B}_{i j}+\mathcal{P}_{i j 0}+\mathcal{A}_{3}(Y)+\mathcal{A}_{4}(Y) \cdot \stackrel{\circ}{B}_{i j} .
$$

Taking the trace of $B_{i j}$ with $h^{i j}$ now yields an expansion for the mean curvature as

$$
H=\left(1+\frac{1}{2} \mathcal{P}_{00}\right) \stackrel{\circ}{H}+\stackrel{i}{h}{ }^{i s} h^{j t} \mathcal{P}_{s t} \stackrel{\circ}{B}_{i j}+\stackrel{\circ}{h}^{i j} \mathcal{P}_{i j 0}+\mathcal{A}_{3}(Y)+\left[\mathcal{A}_{4}(Y)\right]^{i j} \stackrel{\circ}{B}_{i j}
$$

Finally, explicitly expanding the $\mathcal{P}$-quantities in terms of the curvature tensor at the centre of the coordinate chart and estimating the remainder in the same way as before establishes the lemma.

\subsection{Mean curvature calculations in euclidean space}

Let $\Sigma$ be a surface in Euclidean space. Choose a function $f: \Sigma \rightarrow \mathbb{R}$ and define $\check{\mu}_{f}: \Sigma \rightarrow \mathbb{R}^{3}$ as the normal deformation of $\Sigma$ generated by $f$. It is well known that the mean curvature operator $f \mapsto \stackrel{\circ}{H}\left[\stackrel{\circ}{\mu}_{f}(\Sigma)\right]$ with respect to the Euclidean metric decomposes as

$$
\stackrel{\circ}{H}\left[\stackrel{\circ}{\mu}_{f}(\Sigma)\right]=\stackrel{\circ}{H}+\stackrel{\circ}{\mathcal{L}}(f)+\stackrel{\mathcal{Q}}{(f)}
$$

where $\mathcal{L}(f):=\stackrel{\circ}{\mathcal{L}} f+\|\stackrel{\circ}{B}\|^{2} f$ is the linearized mean curvature operator with respect to the Euclidean metric and $\mathcal{Q}(f)$ is the quadratic and higher-order remainder term. We now remind the reader of the expansion for $\mathcal{Q}(f)$ in terms of $f$. Although this result is fairly standard, it is important for our purposes to track the dependence of the various terms in the expansion on $\|\stackrel{B}{\|}\|$.

To begin, let $E_{1}, E_{2}$ be a geodesic normal coordinate frame for $T \Sigma$. Use a comma to denote ordinary differentiation in the coordinate directions. Now introduce the quantities

$$
\begin{aligned}
\beta_{s t} & :=\stackrel{\circ}{h}_{s t}-f \stackrel{\circ}{B}_{s t} \\
\beta^{s t} & :=[\text { Inverse of } \beta]^{s t} \\
D & :=\left(1+\beta^{i k} \beta^{j l} f_{, k} f_{, l} \stackrel{\circ}{h}_{i j}\right)^{1 / 2} .
\end{aligned}
$$

After some work, one finds that the tangent vector fields $\left[E_{f}\right]_{i}$ for $i=1,2$, the components of the Euclidean induced metric $\stackrel{\circ}{h}_{f}$ and its inverse, and the Euclidean 
unit normal vector $\stackrel{\circ}{N}_{f}$ of $\stackrel{\circ}{\mu}_{f}(\Sigma)$ are

$$
\begin{aligned}
{\left[E_{f}\right]_{i} } & :=\stackrel{\circ}{h}^{j k} \beta_{i j} E_{k}+f_{, i} \stackrel{\circ}{\left.\check{h}_{f}\right]_{i j}}:=\beta_{i k} \beta_{j l} \stackrel{\circ}{h}^{k l}+f_{, i} f_{, j} \\
{\left[\stackrel{\circ}{h}_{f}^{-1}\right]^{i j} } & :=\beta^{i m} \beta^{j n}\left(\stackrel{\circ}{h}_{m n}-\frac{\beta^{k p} \beta^{l q} f_{, k} f_{, l} \stackrel{\circ}{h}_{m p} \stackrel{\circ}{h q}_{n}}{D^{2}}\right) \\
\stackrel{\circ}{N}_{f} & :=\frac{1}{D}\left(\stackrel{\circ}{N}-\beta^{i j} f_{, i} E_{j}\right) .
\end{aligned}
$$

Further computations show that the second fundamental form $\stackrel{\circ}{B}_{f}:=\stackrel{\circ}{B}\left[\stackrel{\circ}{\mu}_{f}(\Sigma)\right]$ can be expressed as

$$
D \times\left[\stackrel{\circ}{B}_{f}\right]_{i j}=\stackrel{\circ}{B}_{i j}+f_{; i j}-f \stackrel{\circ}{B}_{i}^{k} \stackrel{\circ}{B}_{k j}+\beta^{k l} f_{, l}\left(f_{, i} \stackrel{\circ}{B}_{j k}+f_{, j} \stackrel{\circ}{B}_{i k}+f \stackrel{\circ}{B}_{j k ; i}\right)
$$

where a semicolon denotes the covariant derivative of $\Sigma$ with respect to $\stackrel{\circ}{h}_{s t}$. We expand the inverse of the induced metric as

$$
\stackrel{\circ}{h}_{f}^{i j}:=\stackrel{\circ}{h}^{i j}+2 f \stackrel{\circ}{B}^{i j}-\frac{1}{D^{2}} \beta^{i m} \beta^{j n} \beta^{k p} \beta^{l q} f_{, k} f_{, l} \stackrel{\circ}{h}_{m p} \stackrel{\circ}{h}_{n q}+\eta^{i m} \eta^{j n} \stackrel{\circ}{h}_{m n}
$$

where the remainder term $\eta^{i j}$ are the components of a tensor defined by $\eta^{i j}:=$ $\beta^{i j}-\stackrel{\circ}{h}^{i j}-f \stackrel{\circ}{B}^{i j}$ which satisfies $\|\eta\|=\mathcal{O}\left(|f|^{2}\|\stackrel{\circ}{B}\|^{2}\right)$. Now taking the trace of (2.7) with respect to $\stackrel{\circ}{h}_{f}^{i j}$ yields the mean curvature $\stackrel{\circ}{H}_{f}:=\stackrel{\circ}{H}_{\left(\mu_{f}\right.}(\Sigma)$ which satisfies

$$
\begin{aligned}
D \times & \stackrel{\circ}{H}_{f} \\
= & \stackrel{\circ}{H}+\stackrel{\circ}{\Delta}+\|\stackrel{\circ}{B}\|^{2} f+2 f \stackrel{\circ}{B}^{i j} f_{; i j}-2 f^{2} \stackrel{\circ}{h}^{i j} \stackrel{\circ}{B}_{i k} \stackrel{\circ}{B}_{l}^{k} \stackrel{\circ}{B}_{j}^{l} \\
+ & \stackrel{\circ}{h}^{i j} \beta^{k l} f_{, l}\left(2 f_{, i} \stackrel{\circ}{B}_{j k}+f \stackrel{\circ}{B}_{j k ; i}\right) \\
+ & \left(-\frac{1}{D^{2}} \beta^{i m} \beta^{j n} \beta^{k p} \beta^{l q} f_{, k} f_{, l} \stackrel{\circ}{h}_{m p} \stackrel{\circ}{h}_{n q}+\eta^{i m} \eta^{j n} \stackrel{\circ}{h}_{m n}\right)\left(\stackrel{\circ}{B}_{i j}+f_{; i j}-f \stackrel{\circ}{B}_{i}^{k} \stackrel{\circ}{B}_{k j}\right) \\
+ & \left(2 f \stackrel{\circ}{B}^{i j}-\frac{1}{D^{2}} \beta^{i m} \beta^{j n} \beta^{k p} \beta^{l q} f_{, k} f_{, l} \stackrel{\circ}{h}_{m p} \stackrel{\circ}{h}_{n q}+\eta^{i m} \eta^{j n} \stackrel{\circ}{h}_{m n}\right) \\
& \cdot \beta^{k l} f_{, l}\left(2 f_{, i} \stackrel{\circ}{B}_{j k}+f \stackrel{\circ}{B}_{j k ; i}\right) .
\end{aligned}
$$

We now easily recognize the terms in the expansion of the mean curvature.

Lemma 2.4. The linear parts of $\stackrel{\circ}{B}_{f}$ and $\stackrel{\circ}{H}_{f}$ are

$$
\begin{aligned}
{\left[\stackrel{\circ}{B}^{(1)}(f)\right]_{i j} } & :=f_{; i j}-f \stackrel{\circ}{B}_{i}^{k} \stackrel{\circ}{B}_{k j} \\
\mathcal{L}(f) & :=\stackrel{\Delta}{\Delta} f+\|\stackrel{\circ}{B}\|^{2} f .
\end{aligned}
$$


The quadratic remainder parts of $\stackrel{\circ}{B}_{f}$ and $\stackrel{\circ}{H}_{f}$ satisfy

$$
\begin{aligned}
& D \times\left[\stackrel{\circ}{B}^{(2)}(f)\right]_{i j}:=\beta^{k l} f_{, l}\left(f_{, i} \stackrel{\circ}{B}_{j k}+f, \stackrel{\circ}{B}_{i k}+f \stackrel{\circ}{B}_{j k ; i}\right) \\
& +(1-D)\left(\stackrel{\circ}{B}_{i j}+f_{; i j}-f \stackrel{\circ}{B}_{i}^{k} \stackrel{\circ}{B}_{k j}\right) \\
& D \times \stackrel{\mathcal{Q}}{(f)}:=2 f \stackrel{\circ}{B}^{i j} f_{; i j}-2 f^{2} \operatorname{Tr}_{\delta}\left(\stackrel{\circ}{B}^{3}\right)+\stackrel{\circ}{h}^{i j} \beta^{k l} f_{, l}\left(2 f_{, i} \stackrel{\circ}{B}_{j k}+f \stackrel{\circ}{B}_{j k ; i}\right) \\
& +\left(-\frac{1}{D^{2}} \beta^{i m} \beta^{j n} \beta^{k p} \beta^{l q} f_{, k} f_{, l} \stackrel{\circ}{h}_{m p} \stackrel{\circ}{h}_{n q}+\eta^{i m} \eta^{j n} \stackrel{\circ}{h}_{m n}\right) \\
& \cdot\left(\stackrel{\circ}{B}_{i j}+f_{; i j}-f \stackrel{\circ}{B}_{i}^{k} \stackrel{\circ}{B}_{k j}\right) \\
& +\left(2 f \stackrel{\circ}{B}^{i j}-\frac{1}{D^{2}} \beta^{i m} \beta^{j n} \beta^{k p} \beta^{l q} f_{, k} f_{, l} \stackrel{\circ}{h}_{m p} \stackrel{\circ}{h}_{n q}+\eta^{i m} \eta^{j n} \stackrel{\circ}{h}_{m n}\right) \\
& \text { - } \beta^{k l} f_{, l}\left(2 f_{, i} \stackrel{\circ}{B}_{j k}+f \stackrel{\circ}{B}_{j k ; i}\right) \\
& +(D-1)\left(\stackrel{\circ}{H}+\stackrel{\circ}{\Delta} f+\|\stackrel{\circ}{B}\|^{2} f\right) \text {. }
\end{aligned}
$$

The quadratic parts of both $\stackrel{\circ}{B}_{f}$ and $\stackrel{\circ}{H}_{f}$ are unwieldy, but only basic structural facts about them are needed in the sequel. To simplify matters, we suppose that $|f|\|\stackrel{\circ}{B}\|+\|\stackrel{\circ}{\nabla} f\| \ll 1$, which will be justified later on. Furthermore, both $\stackrel{\circ}{B}^{(2)}(f)$ and $\mathcal{Q}(f)$ can be expanded into a sum of terms which are each linear combinations of the coefficients of the tensor $f^{i} \cdot\left(\stackrel{\circ}{\nabla}_{f}\right)^{\otimes j} \otimes\left(\stackrel{\circ}{\nabla}^{2} f\right)^{\otimes k} \otimes \stackrel{\circ}{B}^{\otimes l} \otimes(\stackrel{\circ}{\nabla} \stackrel{\circ}{B})^{\otimes m}$, where $i, j, k, l, m$ are positive integers such that $k+m \leq 1$ and $i+1=k+l+2 m$ (i.e. the number of times the function $f$ appears is smaller by one than the sum of the number of covariant derivatives and the number of occurrences of the second fundamental form). Consequently the dominant terms in $\stackrel{\circ}{B}^{(2)}(f)$ and $\stackrel{\mathcal{Q}}{(}(f)$ are $\mathcal{O}(1)$ linear combinations of components of

$(\stackrel{\circ}{\nabla} f)^{2} \otimes \stackrel{\circ}{B}, \quad f \stackrel{\circ}{\nabla} f \otimes \stackrel{\circ}{B}^{2}, \quad f \stackrel{\circ}{\nabla} f \otimes \stackrel{\circ}{\nabla} \stackrel{\circ}{B}, \quad f^{2} \stackrel{\circ}{B}^{3}, \quad f \stackrel{\circ}{\nabla}^{2} f \otimes \stackrel{\circ}{B}$ and $(\stackrel{\circ}{\nabla} f)^{2} \otimes \stackrel{\circ}{\nabla}^{2} f$.

The following estimates are now straightforward consequences of this discussion.

Lemma 2.5. Assuming that $\left|f_{i}\right|\|\stackrel{\circ}{B}\|+\left\|\stackrel{\circ}{\nabla} f_{i}\right\| \ll 1$ for $i=1,2$, the quadratic remainders in the second fundamental form and mean curvature satisfy the following two estimates. First, these operators satisfy the pointwise bounds

$$
\begin{aligned}
& \left|\stackrel{\circ}{B}^{(2)}\left(f_{1}\right)-\stackrel{\circ}{B}^{(2)}\left(f_{2}\right)\right|+\left|\mathcal{Q}\left(f_{1}\right)-\stackrel{\mathcal{Q}}{(}\left(f_{2}\right)\right| \\
& \leq C\left|f_{1}-f_{2}\right| \cdot \max _{i}\left(\left|f_{i}\right|\|\stackrel{\circ}{B}\|^{3}+\left\|\stackrel{\circ}{\nabla} f_{i}\right\|\|\stackrel{\circ}{B}\|^{2}+\left\|\stackrel{\circ}{\nabla} f_{i}\right\|\|\stackrel{\circ}{\nabla}\|+\left\|\stackrel{\circ}{\nabla}^{2} f_{i}\right\|\|\stackrel{\circ}{B}\|\right) \\
& \quad+C\left\|\stackrel{\circ}{\nabla} f_{1}-\stackrel{\circ}{\nabla} f_{2}\right\| \cdot \max _{i}\left(\left|f_{i}\right|\|\stackrel{\circ}{B}\|^{2}+\left\|\stackrel{\circ}{\nabla} f_{i}\right\|\|\stackrel{\circ}{B}\|+\left|f_{i}\right|\|\stackrel{\circ}{\nabla}\|+\left\|\stackrel{\circ}{B} f_{i}\right\|\left\|\stackrel{\circ}{\nabla}^{2} f_{i}\right\|\right) \\
& \quad+C\left\|\stackrel{\circ}{\nabla}^{2} f_{1}-\stackrel{\circ}{\nabla}^{2} f_{2}\right\| \cdot \max _{i}\left(\left|f_{i}\right|\|\stackrel{\circ}{B}\|+\left\|\stackrel{\circ}{\nabla} f_{i}\right\|^{2}\right) .
\end{aligned}
$$


We also have the bounds

$$
\begin{aligned}
& {\left[\stackrel{\circ}{B}^{(2)}\left(f_{1}\right)-\stackrel{\circ}{B}^{(2)}\left(f_{2}\right)\right]_{\alpha}+\left[\stackrel{\mathcal{Q}}{(}\left(f_{1}\right)-\stackrel{\mathcal{Q}}{(}\left(f_{2}\right)\right]_{\alpha}} \\
& \leq C\left[f_{1}-f_{2}\right]_{\alpha} \cdot \max _{i}\left(\left|f_{i}\right|\|\stackrel{\circ}{B}\|^{3}+\left\|\stackrel{\circ}{\nabla} f_{i}\right\|\|\stackrel{\circ}{B}\|^{2}+\left\|\stackrel{\circ}{\nabla} f_{i}\right\|\|\stackrel{\circ}{\nabla} \stackrel{\circ}{B}\|+\left\|\nabla^{2} f_{i}\right\|\|\stackrel{\circ}{B}\|\right) \\
& +C\left[\stackrel{\circ}{\nabla} f_{1}-\stackrel{\circ}{\nabla} f_{2}\right]_{\alpha} \cdot \max _{i}\left(\left|f_{i}\right|\|\stackrel{\circ}{B}\|^{2}+\left\|\stackrel{\circ}{\nabla} f_{i}\right\|\|\stackrel{\circ}{B}\|+\left|f_{i}\right|\|\stackrel{\circ}{\nabla} \stackrel{\circ}{\|}+\| \stackrel{\circ}{\nabla} f_{i}\|\|{ }^{2} f_{i} \|\right) \\
& +C\left[\stackrel{\circ}{\nabla}^{2} f_{1}-\stackrel{\circ}{\nabla}^{2} f_{2}\right]_{\alpha} \cdot \max _{i}\left(\left|f_{i}\right|\|\stackrel{\circ}{B}\|+\left\|\stackrel{\circ}{\nabla} f_{i}\right\|\right) \\
& +C\left|f_{1}-f_{2}\right| \cdot \max _{i}\left(\left[f_{i}\right]_{\alpha}\|\stackrel{\circ}{B}\|^{3}+\left|f_{i}\right|\|\stackrel{\circ}{B}\|^{2}[\stackrel{\circ}{B}]_{\alpha}+\left[\stackrel{\circ}{\nabla} f_{i}\right]_{\alpha}\|\stackrel{\circ}{B}\|^{2}+\left\|\stackrel{\circ}{\nabla} f_{i}\right\|\|\stackrel{\circ}{B}\|[\stackrel{\circ}{B}]_{\alpha}\right. \\
& \left.+\left[\stackrel{\circ}{\nabla} f_{i}\right]_{\alpha}\|\stackrel{\circ}{\nabla} \stackrel{\circ}{B}\|+\left\|\stackrel{\circ}{\nabla} f_{i}\right\|[\stackrel{\circ}{\nabla} \stackrel{\circ}{B}]_{\alpha}+\left[\stackrel{\circ}{\nabla}^{2} f_{i}\right]_{\alpha}\|\stackrel{\circ}{B}\|+\left\|\stackrel{\circ}{\nabla}^{2} f_{i}\right\|[\stackrel{\circ}{B}]_{\alpha}\right) \\
& +C\left\|\stackrel{\circ}{\nabla} f_{1}-\stackrel{\circ}{\nabla} f_{2}\right\| \cdot \max _{i}\left(\left[f_{i}\right]_{\alpha}\|\stackrel{\circ}{B}\|^{2}+\left|f_{i}\right|\|\stackrel{\circ}{B}\|[\stackrel{\circ}{B}]_{\alpha}+\left[\stackrel{\circ}{\nabla} f_{i}\right]_{\alpha}\|\stackrel{\circ}{B}\|+\left\|\stackrel{\circ}{\nabla} f_{i}\right\|[\stackrel{\circ}{B}]_{\alpha}\right. \\
& \left.+\left[f_{i}\right]_{\alpha}\|\stackrel{\circ}{\nabla} \stackrel{\circ}{B}\|+\left|f_{i}\right|[\stackrel{\circ}{\nabla} \stackrel{\circ}{B}]_{\alpha}+\left[\stackrel{\circ}{\nabla} f_{i}\right]_{\alpha}\left\|\stackrel{\circ}{\nabla}^{2} f_{i}\right\|+\left\|\stackrel{\circ}{\nabla} f_{i}\right\|\left[\stackrel{\circ}{ }^{2} f_{i}\right]_{\alpha}\right) \\
& +C\left\|\stackrel{\circ}{\nabla}^{2} f_{1}-\stackrel{\circ}{\nabla}^{2} f_{2}\right\| \cdot \max _{i}\left(\left[f_{i}\right]_{\alpha}\|\stackrel{\circ}{B}\|+\left|f_{i}\right|[\stackrel{\circ}{B}]_{\alpha}+\left[\stackrel{\circ}{\nabla} f_{i}\right]_{\alpha}\left\|\stackrel{\circ}{\nabla} f_{i}\right\|\right)
\end{aligned}
$$

where $C$ is independent of $f_{1}, f_{2}$ and $\|\stackrel{\circ}{B}\|$.

\subsection{Mean curvature calculations for a perturbed background metric}

Consider now a surface $\stackrel{\circ}{\mu}_{f}(\Sigma)$ deformed by the amount $f$ in the direction of the Euclidean normal to $\Sigma$. Working again in a geodesic normal coordinate system centered at some point of $\gamma$, this time we decompose the mean curvature operator $f \mapsto H_{f}:=H\left[\stackrel{\circ}{\mu}_{f}(\Sigma)\right]$ as

$$
H_{f}=\stackrel{\circ}{H}_{f}+\mathcal{H}(f)
$$

where $\stackrel{\circ}{H}_{f}:=\stackrel{\circ}{H}\left[\stackrel{\circ}{\mu}_{f}(\Sigma)\right]$ and $\mathcal{H}(f)$ collects all the terms in the mean curvature $H_{f}$ that depend on the perturbation term $P$ in the background metric $g=\delta+P$ of the coordinate chart. We would like to further expand $\mathcal{H}(f):=\mathcal{H}^{(0)}+\mathcal{H}^{(1)}(f)$ and find a reasonable expression for the latter quantity (from Lemma 2.3 we already know $\mathcal{H}^{(0)}=\mathcal{H}^{(00)}(Y, \stackrel{\circ}{B})+\mathcal{O}\left(\|Y\|^{3}\right)$ provided $\|Y\|\|\stackrel{\circ}{B}\|$ is bounded).

The way to do this is to substitute the quantities given in equation (2.6a) and (2.6b), as well as the position vector field $Y_{f}:=Y+f \stackrel{\circ}{N}$ of $\stackrel{\circ}{\mu}_{f}(\Sigma)$ relative to the center of the normal coordinate chart into the formulæ from Lemma 2.1. Unfortunately, the resulting expressions are extremely unwieldy, but luckily their exact form is not needed in the sequel. We will only need certain estimates.

Proposition 2.6. Let $f_{1}, f_{2}$ satisfy the assumptions $\left|f_{*}\right|\|\stackrel{\circ}{B}\|+\left\|\stackrel{\circ}{\nabla} f_{*}\right\| \ll 1$. Suppose also that $\|Y\|\|\stackrel{B}{B}\|+\|Y\|^{1+\alpha}[\stackrel{\circ}{B}]_{\alpha} \leq C$. Then the operator $\mathcal{H}^{(1)}$ satisfies the 
estimates

$$
\begin{aligned}
& \left|\mathcal{H}^{(1)}\left(f_{1}\right)-\mathcal{H}^{(1)}\left(f_{2}\right)\right| \\
& \leq C\left\|\stackrel{\circ}{B}_{f_{1}}-\stackrel{\circ}{B}_{f_{2}}\right\| \max _{i}\left\|Y_{f_{i}}\right\|^{2} \\
& +C \max _{i}\left\|\stackrel{\circ}{B}_{f_{i}}\right\|\left(\left\|Y_{f_{i}}\right\|\left|f_{1}-f_{2}\right|+\left\|Y_{f_{i}}\right\|^{2}\left(\left|f_{1}-f_{2}\right|\|\stackrel{\circ}{B}\|+\left\|\stackrel{\circ}{\nabla} f_{1}-\stackrel{\circ}{\nabla} f_{2}\right\|\right)\right) \\
& +C \max _{i}\left(\left|f_{1}-f_{2}\right|+\left\|Y_{f_{i}}\right\|\left(\left|f_{1}-f_{2}\right|\|\stackrel{\circ}{B}\|+\left\|\stackrel{\circ}{\nabla} f_{1}-\stackrel{\circ}{\nabla} f_{2}\right\|\right)\right) \\
& {\left[\mathcal{H}^{(1)}\left(f_{1}\right)-\mathcal{H}^{(1)}\left(f_{2}\right)\right]_{\alpha}} \\
& \leq C\left\|\stackrel{\circ}{B}_{f_{1}}-\stackrel{\circ}{B}_{f_{2}}\right\| \max _{i}\left(\left\|Y_{f}\right\|\left[Y_{f}\right]_{\alpha}+\left\|Y_{f}\right\|^{2}\left([f]_{\alpha}\|\stackrel{\circ}{B}\|+|f|[\stackrel{\circ}{B}]_{\alpha}+[\stackrel{\circ}{\nabla} f]_{\alpha}\right)\right) \\
& +C\left[\stackrel{\circ}{B}_{f_{1}}-\stackrel{\circ}{B}_{f_{2}}\right]_{\alpha} \max _{i}\left\|Y_{f_{i}}\right\|^{2} \\
& +C \max _{i}\left[B_{f_{i}}\right]_{\alpha}\left(\left\|Y_{f_{i}}\right\|\left|f_{1}-f_{2}\right|+\left\|Y_{f_{i}}\right\|^{2}\left(\left|f_{1}-f_{2}\right|\|\stackrel{\circ}{B}\|+\left\|\stackrel{\circ}{\nabla} f_{1}-\stackrel{\circ}{\nabla} f_{2}\right\|\right)\right) \\
& +C \max _{i}\left(1+\left\|B_{f_{i}}\right\|\left\|Y_{f_{i}}\right\|\right)\left(\left(\left[f_{i}\right]_{\alpha}\|\stackrel{\circ}{B}\|+\left|f_{i}\right|[\stackrel{\circ}{B}]_{\alpha}+\left[\stackrel{\circ}{\nabla} f_{i}\right]_{\alpha}\right)\left|f_{1}-f_{2}\right|+\left[f_{1}-f_{2}\right]_{\alpha}\right. \\
& +\left(\left[Y_{f_{i}}\right]_{\alpha}+\left\|Y_{f_{i}}\right\|\left(\left[f_{i}\right]_{\alpha}\|\stackrel{\circ}{B}\|+\left|f_{i}\right|[\stackrel{\circ}{B}]_{\alpha}+\left[\stackrel{\circ}{\nabla} f_{i}\right]_{\alpha}\right)\right)\left(\left|f_{1}-f_{2}\right|\|\stackrel{\circ}{B}\|+\left\|\stackrel{\circ}{\nabla} f_{1}-\stackrel{\circ}{\nabla} f_{2}\right\|\right) \\
& \left.+\left\|Y_{f_{i}}\right\|\left(\left[f_{1}-f_{2}\right]_{\alpha}\|\stackrel{\circ}{B}\|+\left|f_{1}-f_{2}\right|[\stackrel{\circ}{B}]_{\alpha}+\left[\stackrel{\circ}{\nabla} f_{1}-\stackrel{\circ}{\nabla} f_{2}\right]_{\alpha}\right)\right)
\end{aligned}
$$

where $C$ is a constant depending only on the geometry of $M$ in the coordinate chart.

Proof. We will use similar notation as Lemma 2.3 , so that $\left[\mathcal{A}_{k}(z)\right]_{k l \ldots}^{i j \cdots}$ indicates a tensor whose components are analytic functions in $z:=\left(z_{1}, z_{2}, z_{3}\right)$ and degree $k$ in $z_{1}$. The mean curvature $H_{f}$ is obtained by substituting $(Y, E, \stackrel{\circ}{N}) \mapsto\left(Y_{f}, E_{f}, \stackrel{\circ}{N}_{f}\right)$ into the $\mathcal{P}$-quantities appearing in the formulæ for $B_{i j}$ and $h_{i j}$ appearing in Lemma 2.1, and then replacing $\stackrel{\circ}{B}_{i j}$ and $\stackrel{\circ}{h}_{i j}$ appearing there by $\left[\stackrel{\circ}{B}_{f}\right]_{i j}$ and $\left[\stackrel{\circ}{h}_{f}\right]_{i j}$. By inspecting the kinds of $\mathcal{P}$-quantities which occur in the resulting expression and using the fact that $h_{f}^{i j}=\stackrel{\circ}{h}_{f}^{i j}+\mathcal{A}_{2}\left(Y_{f}, f \stackrel{\circ}{B}, \stackrel{\circ}{\nabla} f\right)=\stackrel{\circ}{h}^{i j}+\mathcal{A}_{0}\left(Y_{f}, f \stackrel{\circ}{B}, \stackrel{\circ}{\nabla} f\right)$, we see that the operator $\mathcal{H}$ is of the form

$$
\mathcal{H}(f)=\left[\mathcal{A}_{2}\left(Y_{f}, f \stackrel{\circ}{B}, \stackrel{\circ}{\nabla} f\right)\right]^{i j}\left[\stackrel{\circ}{B}_{f}\right]_{i j}+\mathcal{A}_{1}\left(Y_{f}, f \stackrel{\circ}{B}, \stackrel{\circ}{\nabla} f\right) \mid .
$$

To estimate (2.10), we begin with the required estimates for the $\mathcal{A}$-quantities. First, use the fact that $\mathcal{A}_{k}$ has degree $k$ in its first argument and the boundedness of $|f|\|\stackrel{\circ}{B}\|+\|\stackrel{\circ}{\nabla} f\|$ to deduce

$$
\left\|\mathcal{A}_{k}\left(Y_{f}, f \stackrel{\circ}{B}, \stackrel{\circ}{\nabla} f\right)\right\| \leq C\left\|Y_{f}\right\|^{k} .
$$

Next, we deduce from the structure of $\mathcal{A}_{k}(z)$ and $\mathcal{A}_{k}(z)-\mathcal{A}_{k}\left(z^{\prime}\right)$ as analytic functions, as well as the assumptions about $f_{1}, f_{2}$ that

$$
\left[A_{k}\left(Y_{f}, f \stackrel{\circ}{B}, \stackrel{\circ}{\nabla} f\right)\right]_{\alpha} \leq C\left(\left\|Y_{f}\right\|^{k-1}\left[Y_{f}\right]_{\alpha}+\left\|Y_{f}\right\|^{k}\left([f]_{\alpha}\|\stackrel{\circ}{B}\|+|f|[\stackrel{\circ}{B}]_{\alpha}+[\stackrel{\circ}{\nabla} f]_{\alpha}\right)\right)
$$


and

$$
\begin{aligned}
& \left|\mathcal{A}_{k}\left(Y_{f_{1}}, f_{1} \stackrel{\circ}{B}, \stackrel{\circ}{\nabla} f_{1}\right)-\mathcal{A}_{k}\left(Y_{f_{2}}, f_{2} \stackrel{\circ}{B}, \stackrel{\circ}{\nabla} f_{2}\right)\right| \\
& \quad \leq C \max _{i}\left(\left\|Y_{f_{i}}\right\|^{k-1}\left|f_{1}-f_{2}\right|+\left\|Y_{f_{i}}\right\|^{k}\left(\left|f_{1}-f_{2}\right|\|\stackrel{\circ}{B}\|+\left\|\stackrel{\circ}{\nabla} f_{1}-\stackrel{\circ}{\nabla} f_{2}\right\|\right)\right)
\end{aligned}
$$

and

$$
\begin{aligned}
& {\left[A_{k}\left(Y_{f_{1}}, f_{1} \stackrel{\circ}{B}, \stackrel{\circ}{\nabla} f_{1}\right)-\mathcal{A}_{k}\left(Y_{f_{2}}, f_{2} \stackrel{\circ}{B}, \stackrel{\circ}{\nabla} f_{2}\right)\right]_{\alpha}} \\
& \leq \max _{i}\left[\mathcal{A}_{k-1}\left(Y_{f_{i}}, f_{i} \stackrel{\circ}{B}, \stackrel{\circ}{\nabla} f_{i}\right)\right]_{\alpha}\left|f_{1}-f_{2}\right| \\
& +\max _{i}\left|\mathcal{A}_{k-1}\left(Y_{f_{i}}, f_{i} \stackrel{\circ}{B}, \stackrel{\circ}{\nabla} f_{i}\right)\right|\left[f_{1}-f_{2}\right]_{\alpha} \\
& +\max _{i}\left[\mathcal{A}_{k}\left(Y_{f_{i}}, f_{i} \stackrel{\circ}{B}, \stackrel{\circ}{\nabla} f_{i}\right)\right]_{\alpha}\left(\left|f_{1}-f_{2}\right|\|\stackrel{\circ}{B}\|+\left\|\stackrel{\circ}{\nabla} f_{1}-\stackrel{\circ}{\nabla} f_{2}\right\|\right) \\
& +\max _{i}\left|\mathcal{A}_{k}\left(Y_{f_{i}}, f_{i} \stackrel{\circ}{B}, \stackrel{\circ}{\nabla} f_{i}\right)\right|\left(\left[f_{1}-f_{2}\right]_{\alpha}\|\stackrel{\circ}{B}\|+\left|f_{1}-f_{2}\right|[\stackrel{\circ}{B}]_{\alpha}+\left[\stackrel{\circ}{\nabla} f_{1}-\stackrel{\circ}{\nabla} f_{2}\right]_{\alpha}\right) \\
& \leq C \max _{i}\left(\left(\left[Y_{f_{i}}\right]_{\alpha}+\left\|Y_{f_{i}}\right\|^{k-1}\left(\left(\left[f_{i}\right]_{\alpha}\|\stackrel{\circ}{B}\|+\left|f_{i}\right|[\stackrel{\circ}{B}]_{\alpha}+\left[\stackrel{\circ}{\nabla} f_{i}\right]_{\alpha}\right)\left|f_{1}-f_{2}\right|\right.\right.\right. \\
& \left.+\left[f_{1}-f_{2}\right]_{\alpha}\right)+\left\|Y_{f_{i}}\right\|^{k-1}\left(\left[Y_{f_{i}}\right]_{\alpha}+\left\|Y_{f_{i}}\right\|\left(\left[f_{i}\right]_{\alpha}\|\stackrel{\circ}{B}\|+\left|f_{i}\right|[\stackrel{\circ}{B}]_{\alpha}+\left[\stackrel{\circ}{\nabla} f_{i}\right]_{\alpha}\right)\right) \\
& \cdot\left(\left|f_{1}-f_{2}\right|\|\stackrel{\circ}{B}\|+\left\|\stackrel{\circ}{\nabla} f_{1}-\stackrel{\circ}{\nabla} f_{2}\right\|\right) \\
& \left.+\left\|Y_{f_{i}}\right\|^{k}\left(\left[f_{1}-f_{2}\right]_{\alpha}\|\stackrel{\circ}{B}\|+\left|f_{1}-f_{2}\right|[\stackrel{\circ}{B}]_{\alpha}+\left[\stackrel{\circ}{\nabla} f_{1}-\stackrel{\circ}{\nabla} f_{2}\right]_{\alpha}\right)\right) \text {. }
\end{aligned}
$$

We can now estimate

$$
\begin{aligned}
\left|\mathcal{H}\left(f_{1}\right)-\mathcal{H}\left(f_{2}\right)\right| \leq & C\left\|Y_{f_{2}}\right\|^{2}\left\|\stackrel{\circ}{B}_{f_{1}}-\stackrel{\circ}{B}_{f_{2}}\right\|+\| \mathcal{A}_{2}\left(Y_{f_{1}}, f_{1} \stackrel{\circ}{B}, \stackrel{\circ}{\nabla} f_{1}\right) \\
& -\mathcal{A}_{2}\left(Y_{f_{2}}, f_{2} \stackrel{\circ}{B}, \stackrel{\circ}{\nabla} f_{2}\right)\|\| \stackrel{\circ}{B}_{f_{1}} \| \\
& +\left|\mathcal{A}_{1}\left(Y_{f_{1}}, f_{1} \stackrel{\circ}{B}, \stackrel{\circ}{\nabla} f_{1}\right)-\mathcal{A}_{1}\left(Y_{f_{2}}, f_{2} \stackrel{\circ}{B}, \stackrel{\circ}{\nabla} f_{2}\right)\right|
\end{aligned}
$$

and also

$$
\begin{aligned}
{\left[\mathcal{H}\left(f_{1}\right)-\mathcal{H}\left(f_{2}\right)\right]_{\alpha} \leq } & {\left[\mathcal{A}_{2}\left(Y_{f_{2}}, f_{2} \stackrel{\circ}{B}, \stackrel{\circ}{\nabla} f_{2}\right)\right]_{\alpha}\left\|\stackrel{\circ}{B}_{f_{1}}-\stackrel{\circ}{B}_{f_{2}}\right\| } \\
& +\left|\mathcal{A}_{2}\left(Y_{f_{2}}, f_{2} \stackrel{\circ}{\mathrm{B}}, \stackrel{\circ}{\nabla} f_{2}\right)\right|\left[\stackrel{\circ}{B}_{f_{1}}-\stackrel{\circ}{B}_{f_{2}}\right]_{\alpha} \\
& +\left|\mathcal{A}_{2}\left(Y_{f_{2}}, f_{2} \stackrel{\circ}{\mathrm{B}}, \stackrel{\circ}{\nabla} f_{2}\right)-\mathcal{A}_{2}\left(Y_{f_{1}}, f_{1} \stackrel{\circ}{\mathrm{B}}, \stackrel{\circ}{\nabla} f_{1}\right)\right|\left[\stackrel{\circ}{B}_{f_{1}}\right]_{\alpha} \\
& +\left[\mathcal{A}_{2}\left(Y_{f_{2}}, f_{2} \stackrel{\circ}{B}, \stackrel{\circ}{\nabla} f_{2}\right)-\mathcal{A}_{2}\left(Y_{f_{1}}, f_{1} \stackrel{\circ}{\mathrm{B}}, \stackrel{\circ}{\nabla} f_{1}\right)\right]_{\alpha}\left\|\stackrel{\circ}{B} f_{1}\right\| \\
& +\left[\mathcal{A}_{1}\left(Y_{f_{2}}, f_{2} \stackrel{\circ}{\mathrm{B}}, \stackrel{\circ}{\nabla} f_{2}\right)-\mathcal{A}_{1}\left(Y_{f_{1}}, f_{1} \stackrel{\circ}{\mathrm{B}}, \stackrel{\circ}{\nabla} f_{1}\right)\right]_{\alpha} .
\end{aligned}
$$

Once we substitute the appropriate estimates for the $\mathcal{A}$-quantities derived above into these expressions, we obtain the estimates of the proposition since $\mathcal{H}\left(f_{1}\right)-$ $\mathcal{H}\left(f_{2}\right)=\mathcal{H}^{(1)}\left(f_{1}\right)-\mathcal{H}^{(1)}\left(f_{2}\right)$. 


\subsection{Comparison between graphing functions}

In this paper we will consider global normal deformations of a surface $\Sigma$ in $M$ given by a function $f: \Sigma \rightarrow \mathbb{R}$ of the form $\mu_{f}(\Sigma)$, where $\mu$ is the exponential map in the normal direction of $\Sigma$ with respect to the metric of $M$. In order to use the estimates derived in the previous sections, we will need to represent the intersection of $\mu_{f}(\Sigma)$ and a given normal coordinate chart $\mathcal{U}$ as $\stackrel{\circ}{\mu}_{f_{0}}(\Sigma) \cap \mathcal{U}$ where $\stackrel{\leftrightarrow}{\mu}$ is the normal deformation operator with respect the Euclidean metric of the coordinate chart and $f_{0}$ is a different function. We will need the following estimates for $f_{0}$ in the sequel.

Lemma 2.7. Let $\Sigma$ be a surface belonging to a geodesic normal coordinate chart $\mathcal{U} \subseteq M$. Under the assumption that $d:=\operatorname{diam}(\mathcal{U})$ is sufficiently small, and that $|f|\|\stackrel{B}{B}\|+\|\stackrel{\nabla}{ } f\| \ll 1$ on $\Sigma \cap \mathcal{U}$, then there exists a function $f_{0}: \Sigma \rightarrow \mathbb{R}$ so that $\mu_{f}(\Sigma \cap \mathcal{U})=\stackrel{\leftrightarrow}{\mu}_{f_{0}}(\Sigma \cap \mathcal{U})$. Moreover, $f, f_{0}$ satisfy

$$
\sum_{k=0}^{3}\|Y\|^{k}\left\|\nabla^{k} f_{0}-\nabla^{k} f\right\| \leq C d^{2} \sum_{k=0}^{3}\|Y\|^{k}\left\|\nabla^{k} f\right\|
$$

where $C$ is a constant that depends only on the geometry of $M$ and $Y$ is the position vector field inside the coordinate chart.

Proof. Let dist $\left(\cdot, \Sigma^{\prime}\right)$ and $\operatorname{dist}_{0}\left(\cdot, \Sigma^{\prime}\right)$ be the signed distance functions to $\Sigma^{\prime}$ with respect to the metric $g$ and to the Euclidean metric of the coordinate chart, respectively. Let us assume without loss of generality that all distances are positive. Choose $p \in \Sigma \cap \mathcal{U}$ and then $f(p):=\operatorname{dist}\left(p, \mu_{f}(\Sigma)\right)=\operatorname{dist}\left(p, \mu_{f}(p)\right)$ and $f_{0}(p):=\operatorname{dist}_{0}\left(p, \mu_{f}(\Sigma)\right)$ by definition. Define a function $F: \Sigma \cap \mathcal{U} \rightarrow \Sigma \cap \mathcal{U}$ by $f(p):=\operatorname{dist}_{0}\left(p, \mu_{f}(F(p))\right.$ and note that $f_{0}(p)=\left\langle\mu_{f}(F(p))-p, \stackrel{\circ}{N}(p)\right\rangle_{0}$ since $\left\langle\mu_{f}(F(p))-p, X\right\rangle_{0}=0$ for any $X \in T_{p} \Sigma$ by virtue of the fact that $p$ and $\mu_{f}(F(p))$ are at minimum Euclidean distance from each other. Here $\langle\cdot, \cdot\rangle_{0}$ is the Euclidean inner product.

Now $\mu_{f}(q)=\exp _{q}(f(q) N(q))$ where exp is the exponential map with respect to the metric of $M$ and $N(q)$ is the unit normal vector of $\Sigma$ at $q$ with respect to the metric of $M$. Let us write this as $\mu_{f}(q):=G(q, f(q) N(q))$ where $G(q, V):=$ $\exp _{q}(V)$. Since the metric of $M$ and the Euclidean metric satisfy $\max _{i j} \mid g_{i j}-$ $\delta_{i j}\left|+d \max _{i j k}\right| g_{i j, k}\left|+d^{2} \max _{i j k l}\right| g_{i j, k l} \mid \leq C d^{2}$ in the coordinate chart, and the Euclidean-exponential map is $G_{0}(q, V):=q+V$, it can be shown that $G(q, V)=$ $q+V+G_{1}(q, V)$ where $G_{1}(q, V)$ is analytic in its arguments and can be expanded in a Taylor series about the origin of the coordinate chart beginning with quadratic terms and coefficients depending only on the geometry of $M$. Therefore with $q:=$ $F(p)$ we have

$$
\begin{aligned}
f_{0}(p)= & f(q)\langle N(q), \stackrel{\circ}{N}(p)\rangle_{0}+\langle q-p, \stackrel{\circ}{N}(p)\rangle_{0} \\
& +\left\langle G_{1}(q, f(q) N(q)), \stackrel{\circ}{N}(p)\right\rangle_{0} \\
0= & f(q)\langle N(q), X\rangle_{0}+\langle q-p, X\rangle_{0} \\
& +\left\langle G_{1}(q, f(q) N(q)), X\right\rangle_{0} \quad \forall X \in T_{p} \Sigma .
\end{aligned}
$$


The second equation above implies that $\left\|(q-p)^{\|}\right\| \leq C d^{2}|f|_{C^{0}(\mathcal{U})}$ based on our estimates for $G_{1}$ and the comparison estimates from the Section 2.1 for geometric objects with respect to the two different metrics of the coordinate chart. Since $q$ and $p$ both lie on $\Sigma$, we thus get $\left\|(q-p)^{\perp}\right\| \leq C d^{4}|f|_{C^{0}(\mathcal{U})}^{2}\|\stackrel{\circ}{B}\|_{C^{0}(\mathcal{U})}$ and therefore $\|q-p\| \leq C d^{2}|f|_{C^{0}(\mathcal{U})}$ using the assumptions and the comparison estimates of Section 2.1. Hence the first equation above implies

$$
\begin{aligned}
\left|f_{0}(p)-f(p)\right| \leq & |f(p)-f(q)|+|f(q)|\left|\langle N(q), \stackrel{\circ}{N}(p)\rangle_{0}-1\right| \\
& +\left|G_{1}(q, f(q) N(q))\right| \\
\leq & C d^{2}|f|_{C^{0}(\mathcal{U})}
\end{aligned}
$$

again using the assumptions and previous estimates. Finally, the higher derivative estimates follow by differentiating equations (2.11) and proceeding by similar means.

\section{The approximate solutions}

We now construct two families of approximate CMC surfaces. The first family consists of finite-length surfaces invariant under the reflection $t \mapsto-t$ constructed by gluing together $K$ small geodesic spheres of radius $r$ along the $(t, 0,0)$ geodesic with small interpolating necks. Here $K$ is approximately $1 / r$ so that the surface fills out a region along $\gamma$ of bounded length which does not tend to 0 with $r$. The second family consists of one-ended surfaces in an asymptotically flat Riemannian manifold. These are constructed by taking a configuration of $K$ spheres as above and then attaching a semi-infinite Delaunay surface to the last sphere. These two families are denoted $\tilde{\Sigma}_{r}^{F}(\sigma, \delta)$ and $\tilde{\Sigma}_{r}^{O E}(\sigma, \delta)$, respectively. These depend on parameters $\sigma_{1}, \sigma_{2}, \ldots$ and $\delta_{1}, \delta_{2}, \ldots$ which govern the precise locations of the component spheres and necks. For brevity, we will often just write $\tilde{\Sigma}_{r}(\sigma, \delta)$ for either family when the context is clear or does not matter.

\subsection{The finite-length surface}

Let $\gamma$ be the $(t, 0,0)$ geodesic and (with slight abuse of notation) also the arc-length parametrization of this geodesic given by $\gamma(t):=(t, 0,0)$. Introduce the small radius $r$ and the (much smaller) separation parameters $\sigma_{k}$. Let $t_{0}=0$ and $t_{k}:=$ $2 k r+\sum_{l=0}^{k-1}\left(\sigma_{l}\right)$ and let $p_{ \pm k}:=\left( \pm t_{k}, 0,0\right)$. The geodesic spheres that will be used in the gluing construction are $S_{ \pm k}:=\partial B_{r}\left(p_{ \pm k}\right)$. Also let $p_{k}^{b}:=\gamma\left(t_{k}+r+\sigma_{k} / 2\right)$ be the point half-way between $S_{k}$ and $S_{k+1}$ and let $p_{k}^{ \pm}:=\gamma\left(t_{k} \pm r\right)$ be the points in $S_{k} \cap \gamma$. Define $p_{-k}^{ \pm}$and $p_{-k}^{b}$ in a symmetrical manner. In the definitions above, the index $k$ ranges from zero to $K$.

The construction of the first surface consists of three steps. The first step is to replace each $S_{k}$ with $\tilde{S}_{k}$ which is obtained from $S_{k} \backslash\left\{p_{k}^{+}, p_{k}^{-}\right\}$or $S_{0}$ by a small 
normal perturbation designed to make $S_{k}$ look more like a catenoid near $p_{k}^{ \pm}$. The next step is to find the truncated and rescaled catenoids that fit optimally into the space between $\tilde{S}_{k}$ and its neighbours, the precise location of which is governed by displacement parameters $\delta_{k}$. The final step is to use cut-off functions to glue each $\tilde{S}_{k}$ smoothly to its neighbouring necks. The result of this process will be a family of surfaces that depends on $r$ and the parameters $\sigma_{1}, \sigma_{2}, \ldots$ and $\delta_{1}, \delta_{2}, \ldots$ A number of additional small parameters $\varepsilon_{k}^{ \pm}$and $\varepsilon_{k}$ will be introduced below and it will be shown how these depend on the $\sigma$ and $\delta$. Denote by $\varepsilon:=\max _{k}\left\{\varepsilon_{k}, \varepsilon_{k}^{+}, \varepsilon_{k}^{-}\right\}$below and in the rest of the paper.

Step 1. Let $\mathcal{L}_{S}:=\stackrel{\Delta}{\Delta}+\|\stackrel{\circ}{B}\|^{2}$ be the linearized mean curvature operator of $S_{k}$ with respect to the Euclidean metric and let $J_{k}: S_{k} \rightarrow \mathbb{R}$ be the smooth function in the kernel of $\mathcal{L}_{S}$ that is cylindrically symmetric with respect to the axis defined by the geodesic $\gamma$ and normalized to have unit $L^{2}$-norm. (It is defined by taking the correct multiple of the Euclidean normal component of the translation vector field $\frac{\partial}{\partial t}$.)

Now introduce small positive scale parameters $\varepsilon_{k}^{ \pm}$that have yet to be determined and define $G_{k}: S_{k} \backslash\left\{p_{k}^{ \pm}\right\} \rightarrow \mathbb{R}$ for $k=0, \ldots, K-1$ as the unique solution of the equation

$$
\mathcal{L}_{S}\left(G_{k}\right)=\varepsilon_{k}^{+} \delta_{+}+\varepsilon_{k}^{-} \delta_{-}+A_{k} J_{k}
$$

where $\delta_{ \pm}$denotes the Dirac $\delta$-function at $p_{k}^{ \pm}$and the real number $A_{k}$ is chosen to ensure that the right hand side of (3.1a) is $L^{2}$-orthogonal to $J_{k}$. Also, let $G_{0}^{\prime}$ be the unique solution of the equation

$$
\mathcal{L}_{S}\left(G_{K}^{\prime}\right)=\varepsilon_{K}^{-} \delta_{-}+A_{K}^{\prime} J_{K}
$$

where $A_{K}^{\prime}$ is chosen to ensure that the right hand side of (3.1b) is $L^{2}$-orthogonal to $J_{K}$. Note that $\left|A_{k}\right| \leq C \varepsilon$ for $k=1, \ldots, N$ and $A_{0}=0$ by symmetry.

To complete this step, introduce another small radius parameter $r_{k}$ yet to be determined, and define $\tilde{S}_{k}$ as the Euclidean normal graph over $S_{k} \backslash\left[B_{r_{k} / 2}\left(p_{k}^{+}\right) \cup\right.$ $\left.B_{r_{k} / 2}\left(p_{k}^{-}\right)\right]$that is generated by the function $r G_{k}$. Also define the terminal sphere $\tilde{S}_{K}^{\prime}$ as the Euclidean normal graph over $S_{K} \backslash B_{r_{K} / 2}\left(p_{K}^{-}\right)$that is generated by the function $r G_{K}^{\prime}$ as well as its symmetrical counterpart under the $t \mapsto-t$ symmetry.

Step 2. Coordinatize a neighbourhood of $p_{k}^{b}$ using geodesic normal coordinates centered at $p_{k}^{b}$ and scaled by a factor of $r$. Let the coordinate map be $\psi_{k}: B_{R}\left(p_{k}^{b}\right) \subseteq$ $M \rightarrow\left(-R^{\prime}, R^{\prime}\right) \times B_{R^{\prime}}(0) \subseteq \mathbb{R} \times \mathbb{R}^{2}$, where $R, R^{\prime}$ are appropriate radii (one should think of $R=O(r)$ and $R^{\prime}=\mathcal{O}(1)$ ). Note that the $\mathbb{R}$ coordinate corresponds to a translation of the scaled arc-length coordinate along $\gamma$ and $\gamma$ itself maps to the curve $x^{0} \mapsto\left(x^{0}, 0\right)$ with $p_{k}^{b}$ mapping to the origin. The gluing procedure that will now be described applies to any pair of perturbed spheres $\tilde{S}_{k}, \tilde{S}_{k+1}$, including the last pair $\tilde{S}_{K-1}, \tilde{S}_{K}$. The images of $\tilde{S}_{k+1}$ and $\tilde{S}_{k}$ under the coordinate map, at least near the origin, can be represented as graphs over the $\mathbb{R}^{2}$ factor of the form 
$\left\{\left(x^{0}, x\right): x^{0}=F_{\mathrm{sph}}^{ \pm}(x)\right\}$ where + and - correspond to $\psi_{k}\left(\tilde{S}_{k+1}\right)$ and $\psi_{k}\left(\tilde{S}_{k}\right)$ respectively. One can check that the Taylor series expansions for $G_{k}$ near $p_{k}^{ \pm}$and for $\psi_{k}$ imply corresponding expansions for $F_{\mathrm{sph}}^{ \pm}$near $x=0$, which results in the fact that $\psi_{k}\left(\tilde{S}_{k}\right)$ is the set of points

$$
x^{0}=F_{\mathrm{sph}}^{-}(x):=\frac{\sigma_{k}}{2 r}+\varepsilon_{k+1}^{-}\left(c_{k+1}^{-}+C_{k+1}^{-} \log (\|x\|)\right)+\mathcal{O}\left(\|x\|^{2}\right)+\mathcal{O}\left(\varepsilon\|x\|^{2}\right)
$$

while $\psi_{k}\left(\tilde{S}_{k+1}\right)$ is the set of points

$$
x^{0}=F_{\mathrm{sph}}^{+}(x):=-\frac{\sigma_{k}}{2 r}-\varepsilon_{k}^{+}\left(c_{k}^{+}+C_{k}^{+} \log (\|x\|)\right)+\mathcal{O}\left(\|x\|^{2}\right)+\mathcal{O}\left(\varepsilon\|x\|^{2}\right) .
$$

Here, $c_{k}^{ \pm}$and $C_{k}^{ \pm}$are constants.

The interpolation between $\psi_{k}\left(\tilde{S}_{k+1}\right)$ and $\psi_{k}\left(\tilde{S}_{k}\right)$, will be done using a standard catenoid that has been scaled by a factor of $\varepsilon_{k}$ and translated by a small amount along its axis. The $x^{0}>0$ end of such catenoid is given by

$$
\begin{aligned}
x^{0}=F_{\text {neck }}^{+}\left(\varepsilon_{k}, d_{k} ; x\right): & =\varepsilon_{k} \operatorname{arccosh}\left(\|x\| / \varepsilon_{k}\right)+\varepsilon_{k} d_{k} \\
& =\varepsilon_{k}\left(\log (2)-\log \left(\varepsilon_{k}\right)\right)+\varepsilon_{k} \log (\|x\|)+\varepsilon_{k} d_{k}+\mathcal{O}\left(\varepsilon_{k}^{3} /\|x\|^{2}\right)
\end{aligned}
$$

near the origin, where $d_{k}$ is the translation parameter. The $x^{0}<0$ end is given by

$$
\begin{aligned}
x^{0}=F_{\text {neck }}^{-}\left(\varepsilon_{k}, d_{k} ; x\right): & =-\varepsilon_{k} \operatorname{arccosh}\left(\|x\| / \varepsilon_{k}\right)+\varepsilon_{k} d_{k} \\
& =-\varepsilon_{k}\left(\log (2)-\log \left(\varepsilon_{k}\right)\right)-\varepsilon_{k} \log (\|x\|)+\varepsilon_{k} d_{k}+\mathcal{O}\left(\varepsilon_{k}^{3} /\|x\|^{2}\right)
\end{aligned}
$$

near the origin. Optimal matching of these asymptotic expansions of the catenoid with those of $\psi_{k}\left(\tilde{S}_{k+1}\right)$ and $\psi_{k}\left(\tilde{S}_{k}\right)$ given above then requires

$$
\varepsilon_{k}^{+}=\frac{\varepsilon_{k}}{C_{k}^{+}} \quad \varepsilon_{k+1}^{-}=\frac{\varepsilon_{k}}{C_{k+1}^{-}} \quad d_{k}=\frac{1}{2}\left(\frac{c_{k+1}^{-}}{C_{k+1}^{-}}-\frac{c_{k}^{+}}{C_{k}^{+}}\right)
$$

and

$$
\sigma_{k}=\Lambda_{k}\left(\varepsilon_{k}\right):=r \varepsilon_{k}\left(2\left(\log (2)-\log \left(\varepsilon_{k}\right)\right)-\frac{c_{k+1}^{-}}{C_{k+1}^{-}}-\frac{c_{k}^{+}}{C_{k}^{+}}\right) .
$$

These equations imply that once the spacing $\sigma_{k}$ between $\tilde{S}_{k+1}$ and $\tilde{S}_{k}$ has been decided upon, then one can determine $\varepsilon_{k}$ by inverting the equation $\sigma_{k}=\Lambda_{k}\left(\varepsilon_{k}\right)$, and then the other parameters describing the optimally matched neck can be computed from $\varepsilon_{k}$.

Finally, observe that the matching between $\psi_{k}\left(\tilde{S}_{k+1}\right)$ and $\psi_{k}\left(\tilde{S}_{k}\right)$ and the neck defined by the choice of parameters above is most optimal in the region of $x$ where the error quantity $\mathcal{O}\left(\|x\|^{2}\right)+\mathcal{O}\left(\varepsilon_{k}^{3} /\|x\|^{2}\right)$ is smallest. It is easy to check that this occurs when $\|x\|=\mathcal{O}\left(\varepsilon_{k}^{3 / 4}\right)$. Hence the radii $r_{k}$ in Step 1 should be chosen as $r_{k}:=r \varepsilon_{k}^{3 / 4}$, where the factor of $r$ takes the scaling into account. 
Step 3. Choose $d_{k}, \varepsilon_{k}^{ \pm}$and $\varepsilon_{k}$ as above. Also, introduce displacement parameters $\delta_{1}, \delta_{2}, \ldots$ that serve to slightly displace the necks from their optimal locations. Define a smooth, monotone cut-off function $\chi:[0, \infty) \rightarrow[0,1]$ which equals one in $\left[0, \frac{1}{2}\right]$ and vanishes outside $[0,1]$. Define the functions $\tilde{F}^{ \pm}: B_{1}(0) \rightarrow \mathbb{R}$ by

$$
\tilde{F}^{ \pm}(x):=\chi\left(\|x\| / \varepsilon_{k}^{3 / 4}\right) F_{\text {neck }}^{ \pm}\left(\varepsilon_{k}, d_{k}+\delta_{k} ; x\right)+\left(1-\chi\left(\|x\| / \varepsilon_{k}^{3 / 4}\right)\right) F_{\mathrm{sph}}^{ \pm}(x) .
$$

Now define the catenoidal interpolation between $\psi_{k}\left(\tilde{S}_{k+1}\right)$ and $\psi_{k}\left(\tilde{S}_{k}\right)$ as

$$
\tilde{N}_{k}:=\left\{\left(\tilde{F}^{+}(x), x\right): \varepsilon_{k} \leq\|x\| \leq \varepsilon_{k}^{3 / 4}\right\} \cup\left\{\left(\tilde{F}^{-}(x), x\right): \varepsilon_{k} \leq\|x\| \leq \varepsilon_{k}^{3 / 4}\right\} .
$$

Finally, one can define the finite-length approximate solutions as follows.

Definition 3.1. Let $K$ be given. The finite-length surface with parameters $\sigma:=$ $\left\{\sigma_{1}, \ldots, \sigma_{K}\right\}$ and $\delta:=\left\{\delta_{1}, \ldots, \delta_{K}\right\}$ is the surface given by

$$
\begin{aligned}
& \tilde{\Sigma}_{r}^{F}(\sigma, \delta):= {\left[\tilde{S}_{0} \backslash\left[B_{R}\left(p_{0}^{+}\right) \cup B_{R}\left(p_{0}^{-}\right)\right]\right] } \\
& \cup\left[\bigcup_{k=1}^{K-1} \tilde{S}_{k} \backslash\left[B_{R}\left(p_{k}^{+}\right) \cup B_{R}\left(p_{k}^{-}\right)\right]\right] \cup\left[\tilde{S}_{K} \backslash B_{R}\left(p_{K}^{-}\right)\right] \\
& \cup\left[\bigcup_{k=0}^{K-1} \psi_{k}^{-1}\left(\tilde{N}_{k}\right)\right] \\
& \cup \pi\left(\left[\bigcup_{k=1}^{K-1} \tilde{S}_{k} \backslash\left[B_{R}\left(p_{k}^{+}\right) \cup B_{R}\left(p_{k}^{-}\right)\right]\right] \cup\left[\tilde{S}_{K} \backslash B_{R}\left(p_{K}^{-}\right)\right]\right. \\
&\left.\cup\left[\bigcup_{k=0}^{K-1} \psi_{k}^{-1}\left(\tilde{N}_{k}\right)\right]\right) .
\end{aligned}
$$

where $\pi$ is the $t \mapsto-t$ reflection.

\subsection{The one-ended surface}

The one-ended family of approximate solutions is constructed by attaching a half Delaunay surface to a finite-length surface as constructed above. This Delaunay surface has very small necksize parameter, so we will need the detailed analysis of these from [10].

Step 1. Let $K$ be a large integer. Exactly as in the previous section, construct $K$ perturbed spheres of the form $\tilde{S}_{k}$ for $k=1, \ldots, K$ and one terminal perturbed sphere of the form $\tilde{S}_{0}^{\prime}$ (which in this case is a normal graph over $S_{0} \backslash B_{r_{0}}\left(p_{0}^{+}\right)$), along with perturbed necks $\psi_{k}^{-1}\left(\tilde{N}_{k}\right)$ for $k=0, \ldots, K-1$. Then glue these building blocks together using cut-off functions and matched asymptotics again as before. This construction should be equipped with the appropriate separation and displacement parameters $\sigma_{0}, \ldots \sigma_{K-1}$ and $\delta_{0}, \ldots, \delta_{K-1}$. 
Step 2. The standard Delaunay surface of mean curvature 2 and with the appropriate small neck radius can be rescaled by a factor of $r$ and translated along the geodesic $\gamma$ until there is overlap with the last perturbed sphere $\tilde{S}_{K}$ of the construction of Step 1. Optimal overlap can be achieved because the neck region of this Delaunay surface is to first approximation a standard catenoid.

To be a bit more precise with this idea, one proceeds as follows. First recall that Delaunay surfaces of mean curvature 2 are the surfaces of revolution generated by the functions $\rho_{T}: \mathbb{R} \rightarrow \mathbb{R}$ studied in [10, Section 3]. These functions are periodic with period $T$ and have local minima at the integer multiples of $T$. Introduce the parameters $d_{K}$ (which will be fixed once and for all below), and $\delta_{K}$ and $\sigma_{K}$ (which remain free). Define $T:=2+\sigma_{K} / r$ and $\varepsilon_{K}:=\rho_{T}(0)$ and $T_{K}:=t_{K}+r\left(d_{K}+\delta_{K}\right)$ where $t_{K}$ is the arc-length parameter for the center of the perturbed sphere $\tilde{S}_{K}$. Now parametrize a family of translated Delaunay surfaces of mean curvature $\frac{2}{r}$ and period $2 r+\sigma_{K}$ via

$$
\Xi:(t, \theta) \mapsto\left(r \rho_{T}\left(\frac{t-T_{K}}{r}\right) \cos (\theta), r \rho_{T}\left(\frac{t-T_{K}}{r}\right) \sin (\theta), t\right) .
$$

Note that the $t$-parameter now corresponds exactly to the arc-length parameter of $\gamma$. Define truncations of these Delaunay surfaces via

$$
D_{r}^{+}\left(\sigma_{K}, \delta_{K}\right):=\left\{\Xi(t, \theta): t \in\left[T_{K}-\omega, \infty\right) \text { and } \theta \in \mathbb{S}^{1}\right\}
$$

where $\omega$ is a small number of size $\mathcal{O}\left(r \varepsilon^{3 / 4}\right)$.

Next, one must find the optimally matching Delaunay surface. First note that the neck size $\varepsilon_{K}$ of the Delaunay surface $D_{r}^{+}\left(\sigma_{K}, \delta_{K}\right)$ depends on $\sigma_{K}$. Now as in Step 2 of the construction of the finite-length surface, the equality of the constant and logarithmic terms of the expansion of the first neck of $D_{r}^{+}\left(\sigma_{K}, 0\right)$ and of the asymptotic region of $\tilde{S}_{K}$ near $p_{K}^{+}$determines $d_{K}$ and $\varepsilon_{K}^{+}$uniquely in terms of the remaining free parameter $\sigma_{K}$. Now modify $D_{r}^{+}\left(\sigma_{K}, 0\right)$ by means of cut-off function as in Step 3 of of the construction of the finite-length surface so that it coincides exactly with $\tilde{S}_{K}$ in the region $\tilde{S}_{K} \cap\left\{(t, x): \frac{1}{2} r \omega \leq\|x\| \leq r \omega\right\}$. Construct also surfaces where $\delta_{K} \neq 0$, introducing mis-match. Denote the family of modified surfaces by $\tilde{D}_{r}^{+}\left(\sigma_{K}, \delta_{K}\right)$. The definition of the one-ended approximate solutions is finally at hand.

Definition 3.2. Let $K$ be given. The one-ended surface with parameters $\sigma:=$ $\left\{\sigma_{0}, \ldots, \sigma_{K}\right\}$ and $\delta:=\left\{\delta_{0}, \ldots, \delta_{K}\right\}$ is

$$
\tilde{\Sigma}_{r}^{O E}(\sigma, \delta):=\tilde{S}_{0}^{\prime} \backslash B_{R}\left(p_{0}^{+}\right) \cup\left[\bigcup_{k=0}^{K} \tilde{S}_{k} \backslash B_{R}\left(p_{k}^{b}\right)\right] \cup\left[\bigcup_{k=0}^{K-1} \psi_{k}^{-1}\left(\tilde{N}_{k}\right)\right] \cup \tilde{D}_{r}^{+}\left(\sigma_{K}, \delta_{K}\right) \text {. }
$$

\section{Function spaces and norms}

The constant mean curvature equation for normal perturbations of the approximate solutions $\tilde{\Sigma}_{r}(\sigma, \delta)$ constructed in the previous section will be solved for functions in 
weighted Hölder spaces. The weighting will account for the fact that the geometry of $\tilde{\Sigma}_{r}(\sigma, \delta)$ is nearly singular in the small neck-size limit that will be considered here. In fact, two slightly different function spaces will be introduced. The space $C_{v}^{k, \alpha}\left(\tilde{\Sigma}_{r}^{F}(\sigma, \delta)\right)$ will consist of all $C_{\text {loc }}^{k, \alpha}$ functions on $\tilde{\Sigma}_{r}^{F}(\sigma, \delta)$ where the rate of growth in the neck regions of $\tilde{\Sigma}_{r}^{F}(\sigma, \delta)$ is controlled by the parameter $\nu$. The space $C_{\nu, \bar{v}}^{k, \alpha}\left(\tilde{\Sigma}_{r}^{O E}(\sigma, \delta)\right)$ will consist of all $C_{\mathrm{loc}}^{k, \alpha}$ functions on $\tilde{\Sigma}_{r}^{O E}(\sigma, \delta)$ where the rate of growth in the neck regions of $\Sigma_{r}^{O E}(\sigma)$ is controlled by the parameter $v$ and the rate of growth in the asymptotic region along the axis of $\tilde{\Sigma}_{r}^{O E}(\sigma, \delta)$ is controlled by the parameter $\bar{v}$. This latter degree of control is necessitated by the non-compactness of $\tilde{\Sigma}_{r}^{O E}(\sigma, \delta)$. The two types of spaces described above will collectively be denoted $C_{*}^{k, \alpha}\left(\tilde{\Sigma}_{r}(\sigma, \delta)\right)$ for brevity whenever needed.

\subsection{Function spaces and norms for the finite-length surface}

To begin, one must introduce a number of objects. Define a weight function $\zeta_{r}$ : $\tilde{\Sigma}_{r}(\sigma, \delta) \rightarrow \mathbb{R}$ to achieve control of the growth of functions on $\tilde{\Sigma}_{r}(\sigma, \delta)$ in the neck regions:

$$
\zeta_{r}(p):= \begin{cases}r\|x\| & \psi_{k}(p)=(t, x) \in \tilde{N}_{k} \text { with } x \in \bar{B}_{R^{\prime} / 2}(0) \text { for some } k \\ \text { Interpolation } & \psi_{k}(p)=(t, x) \in \psi_{k}\left(\tilde{S}_{k}\right) \text { with } x \in \bar{B}_{R^{\prime}}(0) \backslash B_{R^{\prime} / 2}(0) \\ & \text { for some } k \\ r & \text { elsewhere }\end{cases}
$$

where the interpolation is such that $\zeta_{r}$ is smooth and monotone in the region of interpolation, has appropriately bounded derivatives, and is invariant under all the symmetries of $\tilde{\Sigma}_{r}(\sigma, \delta)$. For the norms themselves, first introduce the following terminology. If $\mathcal{U}$ is any open subset of $\tilde{\Sigma}_{r}(\sigma, \delta)$ and $T$ is any tensor field on $\mathcal{U}$, define

$$
|T|_{0, \mathcal{U}}:=\sup _{x \in \mathcal{U}}\|T(x)\| \quad \text { and } \quad[T]_{\alpha, \mathcal{U}}:=\sup _{x, x^{\prime} \in \mathcal{U}} \frac{\left\|T\left(x^{\prime}\right)-\Xi_{x, x^{\prime}}(T(x))\right\|}{\operatorname{dist}\left(x, x^{\prime}\right)^{\alpha}},
$$

where the norms and the distance function that appear are taken with respect to the induced metric of $\tilde{\Sigma}_{r}(\sigma, \delta)$, while $\Xi_{x, x^{\prime}}$ is the parallel transport operator from $x$ to $x^{\prime}$ with respect to this metric. Then, for any function $f: \mathcal{U} \rightarrow \mathbb{R}$ define

$$
|f|_{k, \alpha, v, \mathcal{U}}:=\sum_{i=0}^{k}\left|\zeta_{r}^{i-v} \nabla^{i} f\right|_{0, \mathcal{U}}+\left[\zeta_{r}^{k+\alpha-v} \nabla^{k} f\right]_{\alpha, \mathcal{U}}
$$

The norms for the finite-length and one-ended surfaces can now be defined.

Define a collection of overlapping open subsets of $\tilde{\Sigma}_{r}^{F}(\sigma, \delta)$ as follows. Let $\bar{R}$ be a fixed radius such that $\bigcup_{k} B_{2 \bar{R}}\left(p_{k}^{b}\right)$ contains all the neck regions of $\tilde{\Sigma}_{r}^{F}(\sigma, \delta)$ and $\mathcal{A}:=\tilde{\Sigma}_{r}^{F}(\sigma, \delta) \backslash\left[\bigcup_{k} \bar{B}_{\bar{R}}\left(p_{k}^{b}\right)\right]$ is the disjoint union of all the spherical regions 
of $\tilde{\Sigma}_{r}(\sigma, \delta)$. Let $\mathcal{A}_{R}:=\tilde{\Sigma}_{r}^{F}(\sigma, \delta) \cap\left[\bigcup_{k} B_{2 R}\left(p_{k}^{\mathrm{b}}\right) \backslash \bar{B}_{R}\left(p_{k}^{\mathrm{b}}\right)\right]$ for any choice of $R \in(0, \bar{R})$.

Definition 4.1. Let $\mathcal{U} \subseteq \tilde{\Sigma}_{r}^{F}(\sigma, \delta)$ and $v \in \mathbb{R}$ and $\alpha \in(0,1)$. The $C_{v}^{k, \alpha}$ norm of a function defined on $\mathcal{U}$ is given by

$$
|f|_{C_{v}^{k, \alpha}(\mathcal{U})}:=|f|_{k, \alpha, \mathcal{U} \cap \mathcal{A}}+\sup _{R \in(0, \bar{R})}|f|_{k, \alpha, \mathcal{U} \cap \mathcal{A}_{R}} .
$$

The function spaces that will be used in the case of the finite-length surface are simply the usual spaces $C^{k, \alpha}\left(\tilde{\Sigma}_{r}^{F}(\sigma, \delta)\right)$, but endowed with the $C_{v}^{k, \alpha}$ norm. This space will be denoted $C_{v}^{k, \alpha}\left(\tilde{\Sigma}_{r}^{F}(\sigma, \delta)\right)$.

\subsection{Function spaces and norms for the one-ended surface}

The definition of the weighted norm that will be used in the case of the one-ended approximate solution builds upon the norm just defined above. Extend the collection of overlapping open subsets used above by defining the points $p_{k}^{b}$ for $k \geq K$ as the points of $\gamma$ upon which the neck regions of $\tilde{D}_{r}^{+}\left(\sigma_{K}, \delta_{K}\right)$ are centered, and then re-defining $\mathcal{A}$ and $\mathcal{A}_{R}$ as infinite unions over all $k \in \mathbb{N}$. Furthermore, re-define the weight function $\zeta_{r}$ by leaving it unchanged on $\tilde{\Sigma}_{r}^{O E}(\sigma, \delta) \backslash \tilde{D}_{r}^{+}\left(\sigma_{K}, \delta_{K}\right)$ and defining

$\zeta_{r}(p):= \begin{cases}r\|x\| & \psi_{k}(p)=(t, x) \in \tilde{D}_{r}^{+}\left(\sigma_{K}, \delta_{K}\right) \text { with } x \in \bar{B}_{R^{\prime} / 2}(0) \\ \text { Interpolation } & \psi_{k}(p)=(t, x) \in \tilde{D}_{r}^{+}\left(\sigma_{K}, \delta_{K}\right) \text { with } x \in \bar{B}_{R^{\prime}}(0) \backslash B_{R^{\prime} / 2}(0) \\ r & \text { elsewhere }\end{cases}$

Finally, for any subset $\mathcal{U} \subseteq \tilde{\Sigma}_{r}^{O E}(\sigma, \delta)$ and function $f \in C_{\text {loc }}^{k, \alpha}(\mathcal{U})$ define the norm $|f|_{k, \alpha, v, \mathcal{U}}$ as above. A second collection of overlapping open subsets of $\tilde{D}_{r}^{+}\left(\sigma_{K}, \delta_{K}\right)$ will be now be introduced. Let $\bar{T}:=t_{K / 2}$ be the arc-length parameter corresponding to the point $p_{N / 2}$ and for any choice of $T \geq \bar{T}$ define $\mathcal{C}_{T}:=$ $\tilde{\Sigma}_{r}^{O E}(\sigma, \delta) \cap\left\{(x, t) \in \mathbb{R}^{2} \times \mathbb{R}: t \in[T, T+r]\right\}$. Also define $\mathcal{C}:=\tilde{\Sigma}_{r}^{O E}(\sigma, \delta) \backslash C_{\bar{T}}$.

Definition 4.2. Let $\mathcal{U} \subseteq \tilde{\Sigma}_{r}^{O E}(\sigma, \delta)$ and $\nu, \bar{v} \in \mathbb{R}$ and $\alpha \in(0,1)$. The $C_{\nu, \bar{v}}^{k, \alpha}$ norm of a function defined on $\mathcal{U}$ is given by

$$
\begin{aligned}
|f|_{C_{v, \bar{v}}^{k, \alpha}(\mathcal{U})}:= & |f|_{k, \alpha, \nu, \mathcal{U} \cap \mathcal{A} \cap \mathcal{C}} \\
& +\sup _{T>\bar{T}} T^{-\bar{v}}\left(|f|_{k, \alpha, \nu, \mathcal{U} \cap \mathcal{A} \cap \mathcal{C}_{T}}+\sup _{R \in\left(0, R_{0}\right)}|f|_{k, \alpha, \nu, \mathcal{U} \cap \mathcal{A}_{R} \cap \mathcal{C}_{T}}\right) .
\end{aligned}
$$

The function spaces that will be used in the case of the one-ended surface are the spaces $C_{\nu, \bar{v}}^{k, \alpha}\left(\tilde{\Sigma}_{r}^{O E}(\sigma, \delta)\right):=\left\{f \in C_{\mathrm{loc}}^{k, \alpha}\left(\tilde{\Sigma}_{r}^{O E}(\sigma, \delta)\right):|f|_{C_{\nu, \bar{v}}^{k, \alpha}}<\infty\right\}$ endowed with the $C_{v, \bar{v}}^{k, \alpha}$ norm. 
Remark 4.3. At first glance, the norm of Definition 4.2 looks different from the norm used to study the linearized mean curvature operator of near-degenerate Delaunay surfaces in [10, Section 4]. This is because the norm in [10, Section 4] is defined using a different parametrization (the $s$-parameter). However, it is straightforward to check, using the estimates of [10, Section 4] relating the $s$-parameter to the arc-length parameter, that the norm in [10, Section 4] is equivalent to the $C_{0, \bar{v}}^{k, \alpha}$ norm defined above.

\section{The solution up to finite-dimensional error}

\subsection{The finite-length surface}

\subsubsection{Strategy}

Let $\mu: C_{\nu}^{2, \alpha}\left(\tilde{\Sigma}_{r}^{F}(\sigma, \delta)\right) \rightarrow \operatorname{Emb}\left(\tilde{\Sigma}_{r}^{F}(\sigma, \delta), M\right)$ be the exponential map of $\tilde{\Sigma}_{r}^{F}(\sigma, \delta)$ in the unit normal direction of $\tilde{\Sigma}_{r}^{F}(\sigma, \delta)$ with respect to the backgroung metric $g$. Hence $\mu_{r f}\left(\tilde{\Sigma}_{r}^{F}(\sigma, \delta)\right)$ is the scaled normal deformation of $\tilde{\Sigma}_{r}(\sigma, \delta)$ generated by $f \in C_{v}^{2, \alpha}\left(\tilde{\Sigma}_{r}^{F}(\sigma, \delta)\right)$. The equation

$$
H\left[\mu_{r f}\left(\tilde{\Sigma}_{r}^{F}(\sigma, \delta)\right)\right]=\frac{2}{r}
$$

selects $f \in C_{v}^{2, \alpha}\left(\tilde{\Sigma}_{r}^{F}(\sigma, \delta)\right)$ so that $\mu_{r f}\left(\tilde{\Sigma}_{r}^{F}(\sigma, \delta)\right)$ has constant mean curvature equal to $\frac{2}{r}$. In addition, the function $f$ will be assumed symmetrical with respect to all the symmetries satisfied by $\tilde{\Sigma}_{r}^{F}(\sigma, \delta)$. Using a fixed-point argument and a suitable choice of weight parameters, the equation (5.1) will be solved up to a finitedimensional error term. That is, we fill find a solution of

$$
H\left[\mu_{r f}\left(\tilde{\Sigma}_{r}^{F}(\sigma, \delta)\right)\right]=\frac{2}{r}+\mathcal{E}
$$

where $\mathcal{E}$ belongs to a finite-dimensional subspace of functions that will be denoted $\tilde{\mathcal{W}}^{F}$ and specified below. At first glance, this error comes from terms in the solution procedure that are not sufficiently small. However, the true reason for the presence of $\mathcal{E}$ is geometric and will be explained in Section 6, where we additionally show how to eliminate it and thus solve equation (5.1) exactly.

To begin, we write $H\left[\mu_{r f}\left(\tilde{\Sigma}_{r}^{F}(\sigma, \delta)\right)\right]:=H\left[\tilde{\Sigma}_{r}(\sigma, \delta)\right]+\mathcal{L}(r f)+$ Pert where $\mathcal{L}:=\Delta+\|B\|^{2}$ and Pert is a perturbation term. Note that $\mathcal{L}$ is not the linearized mean curvature operator of $\tilde{\Sigma}_{r}^{F}(\sigma, \delta)$ since that operator differs from $\mathcal{L}$ by an ambient curvature term. The first step in the solution procedure is to construct a suitably bounded parametrix $\mathcal{R}: C_{\nu-2}^{0, \alpha}\left(\tilde{\Sigma}_{r}^{F}(\sigma, \delta)\right) \rightarrow C_{\nu}^{2, \alpha}\left(\tilde{\Sigma}_{r}^{F}(\sigma, \delta)\right)$ satisfying $\mathcal{L} \circ \mathcal{R}=I d+\mathcal{E}$ where $\mathcal{E}: C_{\nu-2}^{0, \alpha}\left(\tilde{\Sigma}_{r}^{F}(\sigma, \delta)\right) \rightarrow \tilde{\mathcal{W}}^{F}$ is an error term. Now we set $w_{0}:=H\left[\tilde{\Sigma}_{r}^{F}(\sigma, \delta)\right]-\frac{2}{r}$ and use the Ansatz $f:=\frac{1}{r} \mathcal{R}\left(w-w_{0}\right)$ to transform 
equation (5.1) into

$$
w-H\left[\mu_{\mathcal{R}\left(w-w_{0}\right)}\left(\tilde{\Sigma}_{r}^{F}(\sigma, \delta)\right)\right]+\frac{2}{r}+\mathcal{E}\left(w-w_{0}\right)=w+\mathcal{E}\left(w-w_{0}\right) .
$$

Let $\mathcal{N}_{r}: C_{\nu-2}^{0, \alpha}\left(\tilde{\Sigma}_{r}^{F}(\sigma, \delta)\right) \rightarrow C_{\nu-2}^{0, \alpha}\left(\tilde{\Sigma}_{r}^{F}(\sigma, \delta)\right)$ be the non-linear operator given by the left hand side of (5.2). We will show that $\mathcal{N}_{r}$ is a contraction mapping onto a neighbourhood of zero containing $w_{0}$. Once this is done, then we will have solved the equation (5.7) up to the term $\mathcal{E}\left(w-w_{0}\right) \in \tilde{\mathcal{W}}^{F}$.

Showing that $\mathcal{N}_{r}$ is a contraction mapping onto a neighbourhood of zero containing $w_{0}$ is a matter of verifying that two estimates hold when $r$ is sufficiently small. First, that

$$
|w|_{C_{\nu-2}^{0, \alpha}} \leq 2\left|w_{0}\right|_{C_{\nu-2}^{0, \alpha}} \Longrightarrow\left|\mathcal{N}_{r}(w)\right|_{C_{\nu-2}^{0, \alpha}} \leq 2\left|w_{0}\right|_{C_{\nu-2}^{0, \alpha}}
$$

when $r$ is sufficiently small. This establishes that $\mathcal{N}_{r}$ maps a ball of radius $2\left|w_{0}\right|_{C_{\nu-2}^{0, \alpha}}$ into itself. Second, that

$$
|w|_{C_{\nu-2}^{0, \alpha}} \leq 2\left|w_{0}\right|_{C_{\nu-2}^{0, \alpha}} \Longrightarrow\left|\mathcal{N}_{r}\left(w_{1}\right)-\mathcal{N}_{r}\left(w_{2}\right)\right|_{C_{\nu-2}^{0, \alpha}} \leq \frac{1}{2}\left|w_{1}-w_{2}\right|_{C_{\nu-2}^{0, \alpha}}
$$

when $r$ is sufficiently small. This establishes that $\mathcal{N}_{r}$ is a contraction mapping on this ball.

We will apply the following strategy for obtaining the necessary estimates for $\mathcal{N}_{r}$. Since $\tilde{\Sigma}_{r}^{F}(\sigma, \delta)$ is constructed from a number of pieces that are each defined in their own geodesic normal coordinate chart, we will obtain our estimate by patching together estimates in each of these charts. To see how this is done, consider $\mathcal{N}_{r}(w)$ at a point of $\tilde{\Sigma}_{r}^{F}(\sigma, \delta)$ in some geodesic normal coordinate chart $\mathcal{U}$. By Lemma 2.7 we know that we can write $\mu_{f}\left(\tilde{\Sigma}_{r}^{F}(\sigma, \delta) \cap \mathcal{U}\right)=\stackrel{\circ}{\mu}_{f_{0}}\left(\tilde{\Sigma}_{r}^{F}(\sigma, \delta) \cap \mathcal{U}\right)$ which is the normal deformation of $\tilde{\Sigma}_{r}^{F}(\sigma, \delta)$ with respect to the Euclidean metric within the chart by an amount equal to a different function $f_{0}$. Let us view the correspondence $f \mapsto f_{0}$ as having been given by a mapping $\Theta: C_{v}^{2, \alpha}\left(\tilde{\Sigma}_{r}^{F}(\sigma, \delta) \cap \mathcal{U}\right) \rightarrow$ $C_{v}^{2, \alpha}\left(\tilde{\Sigma}_{r}^{F}(\sigma, \delta) \cap \mathcal{U}\right)$. Therefore in $\mathcal{U}$ we can write

$$
\begin{aligned}
& \mathcal{N}_{r}(w)=w-H\left[\stackrel{\circ}{\mu}_{\Theta \circ \mathcal{R}\left(w-w_{0}\right)}\left(\tilde{\Sigma}_{r}^{F}(\sigma, \delta)\right)+\frac{2}{r}+\mathcal{E}\left(w-w_{0}\right)\right.
\end{aligned}
$$

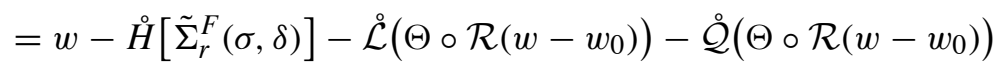

$$
\begin{aligned}
& -\mathcal{H}\left(\Theta \circ \mathcal{R}\left(w-w_{0}\right)\right)+\frac{2}{r}+\mathcal{E}\left(w-w_{0}\right) \\
& \left.=w-\stackrel{\circ}{H}\left[\tilde{\Sigma}_{r}^{F}(\sigma, \delta)\right]-(\stackrel{\circ}{\mathcal{L}} \odot \Theta-\mathcal{L}) \circ \mathcal{R}\left(w-w_{0}\right)\right)-\mathcal{L} \circ \mathcal{R}\left(w-w_{0}\right)
\end{aligned}
$$

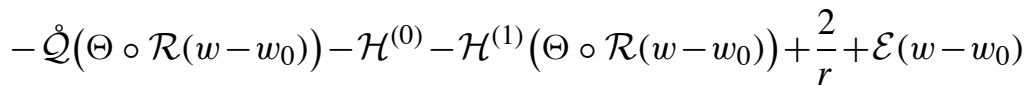

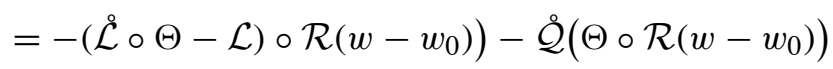

$$
\begin{aligned}
& -\mathcal{H}^{(1)}\left(\Theta \circ \mathcal{R}\left(w-w_{0}\right)\right)
\end{aligned}
$$


using the expansions of the mean curvature in geodesic normal coordinate charts from Section 2. Consequently the estimates (5.3) can be derived by computing five estimates: in Section 5.1.2 we construct and estimate the parametrix $\mathcal{R}$; in Section 5.1.3 we estimate $\left|w_{0}\right|_{C_{v-2}^{0, \alpha}}$; in Section 5.1.4 we estimate the operators $\stackrel{\mathcal{L}}{\circ} \Theta-\mathcal{L}$ and $\mathcal{H}^{(1)}$ and $\mathcal{Q}$; then finally in Section 5.1.5 we put these estimate together to establish that $\mathcal{N}_{r}$ is indeed a contraction mapping on the ball of radius $2\left|w_{0}\right|_{C_{v-2}^{0, \alpha}}$ when $r$ is sufficiently small.

\subsubsection{The linear analysis}

We now construct a parametrix $\mathcal{R}$ satisfying $\mathcal{L} \circ \mathcal{R}=i d+\mathcal{E}$ where $\mathcal{E}$ has finite rank. In each normal coordinate chart, $\mathcal{L}$ is close to the linearized mean curvature operator with respect to the Euclidean metric $\mathcal{L}(f):=\stackrel{\Delta}{ } \dot{\mathcal{L}}\left(f+\|\stackrel{\circ}{B}\|^{2} f\right.$. Patching together inverses for the latter operator gives a parametrix with an error which decomposes into terms which are genuinely small (and thus can be made to vanish after iteration), and those which together constitute $\mathcal{E}$.

We make a few preliminary definitions of the cut-off functions that we will need. First, denote $\operatorname{Ann}_{\tau}(p):=B_{\tau}(p) \backslash B_{\tau / 2}(p)$ and define subsets of $\tilde{\Sigma}_{r}^{F}(\sigma, \delta)$ by

$$
\begin{aligned}
\mathcal{S}_{k}^{\tau}:=\tilde{\Sigma}_{r}^{F}(\sigma, \delta) \backslash\left[B_{\tau}\left(p_{k}^{b}\right) \cup B_{\tau}\left(p_{k-1}^{b}\right)\right] & \mathcal{T}_{k,-}^{\tau}:=\tilde{\Sigma}_{r}^{F}(\sigma, \delta) \cap A n n_{\tau}\left(p_{k-1}^{b}\right) \\
\mathcal{N}_{k}^{\tau}:=\tilde{\Sigma}_{r}^{F}(\sigma, \delta) \cap B_{\tau}\left(p_{k}^{b}\right) & \mathcal{T}_{k,+}^{\tau}:=\tilde{\Sigma}_{r}^{F}(\sigma, \delta) \cap A n n_{\tau}\left(p_{k}^{b}\right) .
\end{aligned}
$$

Now define the smooth, monotone cut-off functions

$$
\begin{aligned}
\chi_{\text {neck }, k}^{\tau}(x) & := \begin{cases}1 & x \in \mathcal{N}_{k}^{\tau} \\
\text { Interpolation } & x \in \mathcal{T}_{k,+}^{\tau} \cup \mathcal{T}_{k+1,-}^{\tau} \\
0 & \text { elsewhere }\end{cases} \\
\chi_{\text {ext }, k}^{\tau}(x) & := \begin{cases}1 & x \in \mathcal{S}_{k}^{\tau} \\
\text { Interpolation } & x \in \mathcal{T}_{k,+}^{\tau} \cup \mathcal{T}_{k,-}^{\tau} \\
0 & \text { elsewhere }\end{cases}
\end{aligned}
$$

so that $\sum_{k} \chi_{\mathrm{ext}, k}^{\tau}+\sum_{k} \chi_{\mathrm{neck}, k}^{\tau}=1$ for all $\tau$. Moreover, let us suppose that these functions are invariant with respect to all symmetries satisfied by $\tilde{\Sigma}_{r}^{F}(\sigma, \delta)$.

Proposition 5.1. Let $v \in(1,2)$. There is an operator $\mathcal{R}: C_{\nu-2}^{0, \alpha}\left(\tilde{\Sigma}_{r}^{F}(\sigma, \delta)\right) \rightarrow$ $C_{v}^{2, \alpha}\left(\tilde{\Sigma}_{r}^{F}(\sigma, \delta)\right)$ that satisfies $\mathcal{L} \circ \mathcal{R}=i d-\mathcal{E}$ where $\mathcal{E}: C_{v-2}^{0, \alpha}\left(\tilde{\Sigma}_{r}^{F}(\sigma, \delta)\right) \rightarrow \tilde{\mathcal{W}}^{F}$. Here $\tilde{\mathcal{W}}^{F}$ is a finite-dimensional space that will be defined below. The estimates satisfied by $\mathcal{R}$ and $\mathcal{E}$ are

$$
|\mathcal{R}(w)|_{C_{\nu}^{2, \alpha}}+|\mathcal{E}(w)|_{C_{2}^{0, \alpha}} \leq C|w|_{C_{\nu-2}^{0, \alpha}}
$$

for all $w \in C_{\nu-2}^{0, \alpha}\left(\tilde{\Sigma}_{r}^{F}(\sigma, \delta)\right)$, where $C$ is a constant independent of $r, \varepsilon$ and $\delta$. 
Proof. Let $w \in C_{v-2}^{0, \alpha}\left(\tilde{\Sigma}_{r}^{F}(\sigma, \delta)\right)$ be given. The task at hand is to solve the equation $\mathcal{L}(u)=w+\mathcal{E}(w)$ for a function $u \in C_{v}^{2, \alpha}\left(\tilde{\Sigma}_{r}^{F}(\sigma, \delta)\right)$ and an error term $\mathcal{E}(w) \in$ $\tilde{\mathcal{W}}^{F}$. To begin, introduce four radii $\tau_{1}<\tau_{2}<\tau_{3}<\tau_{4} \ll r$ with the property that the supports of the gradients of the cut-off functions $\chi_{*}^{\tau_{i}}$ and $\chi_{*}^{\tau_{j}}$ do not overlap for $i \neq j$. These radii will be further specified below.

Step 1. Let $w_{\text {neck, } k}:=w \chi_{\text {neck }, k}^{\tau_{3}}$. This function has compact support in $\mathcal{N}_{k}^{\tau_{3}}$ and thus can be viewed as a function of compact support on the standard scaled catenoid. Now consider the equation $\mathcal{L}_{N}(u)=w_{\text {neck, } k}$ on the standard scaled catenoid, where $\mathcal{L}_{N}$ is the linearized mean curvature operator of the scaled catenoid $r \varepsilon_{k} N$ with respect to the Euclidean metric. Then the pull-back of $\mathcal{L}$ to the standard scaled catenoid is a small perturbation of $\mathcal{L}_{N}$. By the theory of the Laplace operator on asymptotically flat manifolds, the operator $\mathcal{L}_{N}$ is surjective onto $C_{v-2}^{0, \alpha}\left(r \varepsilon_{k} N\right)$ when $v \in(1,2)$. Hence there is a solution $u_{\text {neck, } k}$ as desired, satisfying the estimate $\left|u_{\text {neck }, k}\right|_{C_{v}^{2, \alpha}} \leq C|w|_{C_{\nu-2}^{0, \alpha}}$ where these norms can be taken as the pull-backs of the weighted Hölder norms being used to measure functions on $\tilde{\Sigma}_{r}^{F}(\sigma, \delta)$. Finally, extend $u_{\text {neck, } k}$ to all of $\tilde{\Sigma}_{r}^{F}(\sigma, \delta)$ by the definition $\bar{u}_{\text {neck, } k}:=u_{\text {neck, } k} \chi_{\text {neck, } k}^{\tau_{4}}$ and set $\bar{u}_{\text {neck }}:=\sum_{k} \bar{u}_{\text {neck }, k}$.

Step 2. Define $w_{\text {ext }, k}:=\left(w-\mathcal{L}\left(\bar{u}_{\text {neck }}\right)\right) \chi_{\text {ext }, k}^{\tau_{2}}$. Then $w_{\text {ext }, k}$ is a function of compact support on $\mathcal{S}_{k}^{\tau_{2}}$ and thus can be viewed as a function of compact support on the sphere $S_{k} \backslash\left\{p_{k}^{+}, p_{k}^{-}\right\}$. Now consider the equation $\mathcal{L}_{S}(u)=w_{\text {ext }, k}$, where $\mathcal{L}_{S}$ is the linearized mean curvature operator of the sphere of radius $r$ with respect to the Euclidean metric. Then the pull-back of $\mathcal{L}$ to the sphere is a small perturbation of $\mathcal{L}_{S}$. It is not a priori possible to solve the equation $\mathcal{L}_{S}\left(u_{\text {ext }, k}\right)=w_{\text {ext }, k}$ on $S_{k}$ because of the one-dimensional kernel of $\mathcal{L}_{S}$ that is invariant with respect to the symmetries that have been imposed. A basis for the kernel is given by the function $J_{k}: S_{k} \rightarrow \mathbb{R}$ defined by $J_{k}:=\stackrel{\circ}{g}\left(\stackrel{\circ}{N}, \frac{\partial}{\partial t}\right)$ where $\stackrel{\circ}{g}$ is the Euclidean metric and $\stackrel{\circ}{N}$ is the unit normal vector of $S_{k}$. Let $w_{\mathrm{ext}, k}^{\perp}:=w_{\mathrm{ext}, k}-\left\langle w_{\mathrm{ext}, k}, J_{k}\right\rangle J_{k}$ be the projection of $w_{\mathrm{ext}, k}$ to the orthogonal complement of $\operatorname{span}\left\{J_{k}\right\}$ with respect to the Euclidean $L^{2}$-inner product denoted by $\langle\cdot, \cdot\rangle$. Now there is a solution of the equation $\mathcal{L}_{S}\left(u_{\mathrm{ext}, k}\right)=w_{\mathrm{ext}, k}^{\perp}$ satisfying the estimate $\left|u_{\mathrm{ext}, k}\right|_{C^{2, \alpha}}^{*} \leq C\left|w_{\mathrm{ext}, k}^{\perp}\right|_{C^{0, \alpha}}^{*}$ where $|\cdot|_{C^{k, \alpha}}^{*}$ is the scale-invariant $C^{k, \alpha}$ norm where derivatives and the Hölder coefficient are weighted by appropriate factors of $r$. Furthermore, one has $\left|w_{\mathrm{ext}, k}^{\perp}\right|_{C^{0, \alpha}}^{*} \leq C\left|w_{\mathrm{ext}, k}\right|_{C^{0, \alpha}}^{*} \leq C \tau_{2}^{\nu-2}|w|_{C_{v-2}^{0, \alpha}}^{*}$.

A modified solution satisfying weighted estimates can be obtained as follows. First, write $u_{\mathrm{ext}, k}:=v_{\mathrm{ext}, k}+a_{k}^{+} \eta_{k}^{+}+a_{k}^{-} \eta_{k}^{-}$where $v_{\mathrm{ext}, k}$ satisfies $\left|v_{\mathrm{ext}, k}(x)\right| \leq$ $C \tau_{2}^{\nu-2} \operatorname{dist}\left(x, p_{k}^{*}\right)^{2}|w|_{C_{\nu-2}^{0, \alpha}}$ near $p_{k}^{*}$, while the function $\eta_{k}^{ \pm}$equals one near $p_{k}^{ \pm}$and vanishes a small but $\varepsilon$-independent distance away from these points, and $a^{ \pm} \in$ $\mathbb{R}$ satisfies $\left|a^{ \pm}\right| \leq C \tau_{2}^{\nu-2}|w|_{C_{\nu-2}^{0, \alpha}}$. This decomposition is achieved by studying the Taylor series expansion of $u_{\text {ext }, k}$ near $p_{k}^{ \pm}$and using the symmetries satisfied by $u_{\text {ext, } k}$. Finally, by adding the correct multiple of $J_{k}$ to $u_{\text {ext, } k}$ on each $S_{k}$, one 
can arrange to have $a_{k}^{-}=0$ for every $k \geq 1$ and $a_{0}^{ \pm}=0$ by symmetry. To extend the functions $u_{\text {ext }, k}$ to all of $\tilde{\Sigma}_{r}^{F}(\sigma, \delta)$, define $\bar{u}_{\text {ext }}:=\bar{v}_{\text {ext }}+A$ where $\bar{v}_{\text {ext }}:=$ $\sum_{k} \chi_{\mathrm{ext}, k}^{\tau_{1}} v_{\mathrm{ext}, k}$ and $A:=\sum_{k} a_{k}^{+} \eta_{k}^{+} \chi_{\text {neck }, k}^{\tau_{1}}$. Then one has the estimate $\left|\bar{v}_{\mathrm{ext}}\right|_{C_{v}^{2, \alpha}}+$ $|A|_{C_{v}^{2, \alpha}} \leq C \tau_{2}^{\nu-2}|w|_{C_{v-2}^{0, \alpha}}$.

Step 3. Let $u^{(1)}:=\bar{v}_{\text {ext }}+\bar{u}_{\text {neck }}$ and $\mathcal{E}^{(1)}(w):=-\sum_{k} \chi_{\text {ext }, k}^{\tau_{1}}\left\langle w_{\text {ext }, k}, J_{k}\right\rangle J_{k}-$ $\sum_{k} \chi_{\text {ext }, k}^{\tau_{1}} \mathcal{L}_{S}(A)$. By collecting the estimates from Steps 1 and 2 , one has $\left|u^{(1)}\right|_{C_{v}^{2, \alpha}} \leq$ $C|w|_{C_{\nu-2}^{0, \alpha}}$. The claim is that

$$
\left|\mathcal{L}\left(u^{(1)}\right)-w-\mathcal{E}^{(1)}(w)\right|_{C_{\nu-2}^{0, \alpha}} \leq \theta|w|_{C_{\nu-2}^{0, \alpha}} \quad \text { and } \quad\left|\mathcal{E}^{(1)}(w)\right|_{C_{2}^{0, \alpha}} \leq C \tau_{2}^{\nu-2}|w|_{C_{\nu-2}^{0, \alpha}}
$$

where $\theta$ can be made as small as desired by adjusting $\tau_{1}, \ldots, \tau_{4}$ and $\varepsilon$ suitably. The consequence is that one can iterate Steps 1 and 2 to construct sequences $u^{(n)}$ and $\mathcal{E}^{(n)}(w)$ that converge to $u:=\mathcal{R}(w)$ and $\mathcal{E}(w)$ respectively, satisfying the desired bounds.

Therefore to complete the proof of the proposition, it remains to compute the estimates given in 5.5. The idea is to exploit the fact that $\mathcal{L}$ differs very little from $\mathcal{L}_{N}$ and $\mathcal{L}_{S}$ in the regions where $u^{(1)}$ equals $\bar{v}_{\text {ext }}$ and $\bar{u}_{\text {neck }}$, all while taking into account the effects of the cut-off functions. With this in mind, and using the notation $[\mathcal{L}, \chi](u):=\mathcal{L}(\chi u)-\chi \mathcal{L}(u)$, one has

$$
\begin{aligned}
& \mathcal{L}\left(u^{(1)}\right)=\left(\mathcal{L}-\mathcal{L}_{S}\right)\left(\bar{v}_{\text {ext }}\right)+\sum_{k}\left[\mathcal{L}_{S}, \chi_{\text {ext }, k}^{\tau_{1}}\right]\left(v_{\text {ext }, k}\right)+\sum_{k} \chi_{\text {ext }, k}^{\tau_{1}} \mathcal{L}_{S}\left(v_{\text {ext }, k}\right)+\mathcal{L}\left(\bar{u}_{\text {neck }}\right) \\
& =\left(\mathcal{L}-\mathcal{L}_{S}\right)\left(\bar{v}_{\text {ext }}\right)+\sum_{k}\left[\mathcal{L}_{S}, \chi_{\text {ext }, k}^{\tau_{1}}\right]\left(v_{\text {ext }, k}\right)+\sum_{k} \chi_{\text {ext }, k}^{\tau_{1}} w_{\text {ext }, k} \\
& -\sum_{k} \chi_{\mathrm{ext}, k}^{\tau_{1}}\left\langle w_{\mathrm{ext}, k}, J_{k}\right\rangle J_{k}-\sum_{k} \chi_{\mathrm{ext}, k}^{\tau_{1}} \mathcal{L}_{S}(A)+\mathcal{L}\left(\bar{u}_{\text {neck }}\right) \\
& =\left(\mathcal{L}-\mathcal{L}_{S}\right)\left(\bar{v}_{\text {ext }}\right)+\sum_{k}\left[\mathcal{L}_{S}, \chi_{\text {ext }, k}^{\tau_{1}}\right]\left(v_{\text {ext }, k}\right)+\sum_{k} \chi_{\text {ext }, k}^{\tau_{2}}\left(w-\mathcal{L}\left(\bar{u}_{\text {neck }}\right)\right) \\
& -\sum_{k} \chi_{\mathrm{ext}, k}^{\tau_{1}}\left\langle w_{\mathrm{ext}, k}, J_{k}\right\rangle J_{k}-\sum_{k} \chi_{\mathrm{ext}, k}^{\tau_{1}} \mathcal{L}_{S}(A)+\mathcal{L}\left(\bar{u}_{\text {neck }}\right) \\
& =\left(\mathcal{L}-\mathcal{L}_{S}\right)\left(\bar{v}_{\text {ext }}\right)+\sum_{k}\left[\mathcal{L}_{S}, \chi_{\text {ext }, k}^{\tau_{1}}\right]\left(v_{\text {ext }, k}\right)+\sum_{k} \chi_{\text {ext }, k}^{\tau_{2}} w+\sum_{k} \chi_{\text {neck }, k}^{\tau_{2}} \mathcal{L}\left(\bar{u}_{\text {neck }}\right) \\
& -\sum_{k} \chi_{\mathrm{ext}, k}^{\tau_{1}}\left\langle w_{\mathrm{ext}, k}, J_{k}\right\rangle J_{k}-\sum_{k} \chi_{\mathrm{ext}, k}^{\tau_{1}} \mathcal{L}_{S}(A) \\
& =\left(\mathcal{L}-\mathcal{L}_{S}\right)\left(\bar{v}_{\text {ext }}\right)+\sum_{k}\left[\mathcal{L}_{S}, \chi_{\text {ext }, k}^{\tau_{1}}\right]\left(v_{\text {ext }, k}\right)+\sum_{k} \chi_{\text {neck }, k}^{\tau_{2}}\left(\mathcal{L}-\mathcal{L}_{N}\right)\left(\bar{u}_{\text {neck }}\right) \\
& -\sum_{k} \chi_{\mathrm{ext}, k}^{\tau_{1}}\left\langle w_{\mathrm{ext}, k}, J_{k}\right\rangle J_{k}-\sum_{k} \chi_{\mathrm{ext}, k}^{\tau_{1}} \mathcal{L}_{S}(A)+w
\end{aligned}
$$

since $\mathcal{L}\left(\bar{u}_{\text {neck }}\right)=\left(\mathcal{L}-\mathcal{L}_{N}\right)\left(\bar{u}_{\text {neck }}\right)+\sum_{k}\left[\mathcal{L}_{N}, \chi_{\text {neck }, k}^{\tau_{4}}\right]\left(u_{\text {neck }, k}\right)+\sum_{k} \chi_{\text {neck }, k}^{\tau_{4}} \mathcal{L}_{N}\left(u_{\text {neck }, k}\right)$. The facts $\chi_{\mathrm{ext}, k}^{\tau_{1}} \quad \chi_{\mathrm{ext}, k}^{\tau_{2}}=\chi_{\mathrm{ext}, k}^{\tau_{2}}$ and $\chi_{\text {neck, } k}^{\tau_{2}} \quad \chi_{\text {neck, }, k}^{\tau_{3}} \quad \chi_{\text {neck, } k}^{\tau_{4}}=\chi_{\text {neck, }, k}^{\tau_{2}}$ and 
$\chi_{\text {neck }, k}^{\tau_{2}}\left[\mathcal{L}_{N}, \chi_{\text {neck, } k}^{\tau_{4}}\right]=0$ have also been used in the calculations above. The claim now follows since both $\mathcal{L}-\mathcal{L}_{S}$ and $\mathcal{L}-\mathcal{L}_{N}$ can be handled using Lemma 2.2 while

$$
\left|\left[\mathcal{L}_{S}, \chi_{\mathrm{ext}, k}^{\tau_{1}}\right] v_{\mathrm{ext}, k}\right|_{C_{v-2}^{0, \alpha}} \leq C\left|v_{\mathrm{ext}, k}\right|_{C_{v}^{2, \alpha}\left(\operatorname{supp}\left(\nabla \chi_{\mathrm{ext}, k}^{\tau_{1}}\right)\right)} \leq C\left(\frac{\tau_{1}}{\tau_{2}}\right)^{2-v}|w|_{C_{v-2}^{0, \alpha}}
$$

can be made as small as desired by adjusting the ratio $\tau_{1} / \tau_{2}$.

The iteration leading to the exact solution of the equation $\mathcal{L}(u)=w+\mathcal{E}(w)$ now works as follows. Using the steps above, for every $n \geq 0$ one has functions $u^{(n)} \in C_{v}^{2, \alpha}\left(\tilde{\Sigma}_{r}^{F}(\sigma, \delta)\right)$ and $w^{(n)} \in C_{\nu-2}^{0, \alpha}\left(\tilde{\Sigma}_{r}^{F}(\sigma, \delta)\right)$ satisfying $\mathcal{L}\left(u^{(n)}\right)=w^{(n-1)}+$ $\mathcal{E}\left(u^{(n-1)}\right)+w^{(n)}$ and $w^{(0)}=w$ along with the estimates

$$
\left|u^{(n)}\right|_{C_{\nu}^{2, \alpha}}+\left|\mathcal{E}\left(w^{(n-1)}\right)\right|_{C_{0}^{2, \alpha}} \leq C\left|w^{(n-1)}\right|_{C_{\nu-2}^{0, \alpha}} \text { and } \quad\left|w^{(n)}\right|_{C_{\nu-2}^{0, \alpha}} \leq \frac{1}{2}\left|w^{(n-1)}\right|_{C_{\nu-2}^{0, \alpha}} \text {. }
$$

Consequently the series $u:=\sum_{n=1}^{\infty}(-1)^{n+1} u^{(n)}$ and $\mathcal{E}(w):=\sum_{n=1}^{\infty}(-1)^{n+1} \mathcal{E}\left(w^{(n-1)}\right)$ converge in the appropriate norms and satisfy $\mathcal{L}(u)=w+\mathcal{E}(w)$ along with the desired estimates.

The definition of the finite-dimensional image of the map $\mathcal{E}: C_{\nu-2}^{0, \alpha}\left(\tilde{\Sigma}_{r}^{F}(\sigma, \delta)\right) \rightarrow$ $\tilde{\mathcal{W}}^{F}$ is a by-product of Step 3 of the previous proof.

Definition 5.2. Define

$$
\tilde{\mathcal{W}}^{F}:=\operatorname{span}\left\{\chi_{\mathrm{ext}, k}^{\tau_{1}} J_{k}, \chi_{\mathrm{ext}, k}^{\tau_{1}} \mathcal{L}_{S}\left(\eta_{k}^{+} \chi_{\text {neck }, k}^{\tau_{1}}\right): k=1, \ldots, K-1\right\} \cup\left\{\chi_{\mathrm{ext}, K}^{\tau_{1}} J_{K}\right\} .
$$

\subsubsection{The mean curvature estimate}

We next estimate the quantity $w_{0}:=H\left[\tilde{\Sigma}_{r}^{F}(\sigma, \delta)\right]-\frac{2}{r}$ in the $C_{\nu-2}^{0, \alpha}$ norm when $v \in(1,2)$. In the following, set $\varepsilon:=\max \left\{\varepsilon_{k}^{ \pm}, \varepsilon_{k}\right\}$ and $\delta:=\max \left\{\delta_{k}\right\}$ and recall that $r_{k}=\mathcal{O}\left(r \varepsilon^{3 / 4}\right)$.

Proposition 5.3. Suppose $v \in(1,2)$. The mean curvature of $\tilde{\Sigma}_{r}^{F}(\sigma, \delta)$ satisfies the estimate

$$
\left|H\left[\tilde{\Sigma}_{r}^{F}(\sigma, \delta)\right]-\frac{2}{r}\right|_{C_{v-2}^{0, \alpha}} \leq C \max \left\{r^{3-v}, r^{1-v} \varepsilon^{3 / 2-3 v / 4}, \delta r^{1-v} \varepsilon^{1-3 v / 4}\right\}
$$

for some constant $C$ independent of $r, \varepsilon, \delta$ and $K$.

Proof. Since we can write $H\left[\tilde{\Sigma}_{r}^{F}(\sigma, \delta)\right]$ in terms of $\stackrel{\circ}{H}\left[\tilde{\Sigma}_{r}^{F}(\sigma, \delta)\right]$ and $\stackrel{\circ}{B}\left[\tilde{\Sigma}_{r}^{F}(\sigma, \delta)\right]$ and quantities depending on the background geometry as in Section 2.1, we can break up the estimate of $H\left[\tilde{\Sigma}_{r}^{F}(\sigma, \delta)\right]$ into several steps. The first two steps are to derive pointwise estimates for $\stackrel{\circ}{H}\left[\tilde{\Sigma}_{r}^{F}(\sigma, \delta)\right]$ and $\stackrel{\circ}{B}\left[\tilde{\Sigma}_{r}^{F}(\sigma, \delta)\right]$ with respect to the Euclidean background metric of the geodesic normal coordinate charts used to define the spherical and neck regions, respectively; the third step takes into account the contribution from the actual background metric in each coordinate chart; and the fourth step is to compute the desired $C_{\nu-2}^{0, \alpha}$ norms of $H\left[\tilde{\Sigma}_{r}^{F}(\sigma, \delta)\right]-\frac{2}{r}$. Finally, we note that all estimates computed below are independent of $K$. 
Step 1. The first step it to find pointwise estimates for $\stackrel{\circ}{B}:=\stackrel{\circ}{B}\left[\tilde{\Sigma}_{r}^{F}(\sigma, \delta)\right]$ and $\stackrel{\circ}{H}:=\stackrel{\circ}{H}\left[\tilde{\Sigma}_{r}^{F}(\sigma, \delta)\right]$ in the coordinate chart used to define the spherical region $\tilde{S}_{k}$ of $\tilde{\Sigma}_{r}^{F}(\sigma, \delta)$. The key to this estimate is to use the formulæ (2.7) and (2.8) for $\stackrel{\circ}{B}$ and $\stackrel{\circ}{H}$ in terms of the graphing function $r G$ and the second fundamental form $\stackrel{B}{B}\left(S_{k}\right)$ and mean curvature $\stackrel{H}{H}\left(S_{k}\right)$ of $S_{k}$ with respect to the Euclidean metric in conjunction with the estimates from Lemma 2.5. The results are

$$
\begin{aligned}
& \|\stackrel{\circ}{B}\|+r^{\alpha}[\stackrel{\circ}{B}]_{\alpha} \\
& \leq C\left(\left\|\stackrel{\circ}{B}\left(S_{k}\right)\right\|+r\left\|\stackrel{\circ}{ }^{2} G\right\|+r\left(|G|+r^{\alpha}[G]_{\alpha}\right)\left\|\stackrel{\circ}{B}\left(S_{k}\right)\right\|^{2}+r^{\alpha}\left[\stackrel{\circ}{ }^{2} G\right]_{\alpha}\right) \\
& \leq \frac{C}{r}\left(1+|G|+r\|\stackrel{\circ}{\nabla} G\|+r^{2}\left\|\stackrel{\circ}{ }^{2} G\right\|+r^{2+\alpha}\left[\stackrel{\circ}{\nabla}^{2} G\right]_{\alpha}\right)
\end{aligned}
$$

and

$$
\begin{aligned}
& \left|\stackrel{\circ}{H}-\frac{2}{r}\right|+r^{\alpha}[\stackrel{\circ}{H}]_{\alpha} \\
& \leq C r^{2}\left(|G|^{2}\left\|\stackrel{\circ}{B}\left(S_{k}\right)\right\|^{3}+|G|\left\|\nabla^{\circ} G\right\|\left\|\stackrel{\circ}{B}\left(S_{k}\right)\right\|^{2}+|G|\left\|\nabla^{2} G\right\|\left\|\stackrel{\circ}{ }\left(S_{k}\right)\right\|\right. \\
& \left.+\|\stackrel{\circ}{\nabla} G\|^{2}\left\|\stackrel{\circ}{B}\left(S_{k}\right)\right\|+r\|\stackrel{\circ}{\nabla} G\|^{2}\left\|\stackrel{\circ}{\nabla}^{2} G\right\|\right) \\
& +C r^{2+\alpha}\left([G]_{\alpha}\left(|G|\left\|\stackrel{\circ}{B}\left(S_{k}\right)\right\|^{3}+\|\stackrel{\circ}{\nabla} G\|\left\|\stackrel{\circ}{B}\left(S_{k}\right)\right\|^{2}+\left\|\stackrel{\circ}{ }^{2} G\right\|\left\|\stackrel{\circ}{B}\left(S_{k}\right)\right\|\right)\right. \\
& +[\stackrel{\circ}{\nabla} G]_{\alpha}\left(|G|\left\|\stackrel{\circ}{B}\left(S_{k}\right)\right\|^{2}+\|\stackrel{\circ}{\nabla} G\|\left\|\stackrel{\circ}{B}\left(S_{k}\right)\right\|+r\left\|\stackrel{\circ}{\nabla}^{2} G\right\|\|\stackrel{\circ}{\nabla} G\|\right) \\
& +\left[\stackrel{\circ}{\nabla}^{2} G\right]_{\alpha}\left(|G|\left\|\stackrel{\circ}{B}\left(S_{k}\right)\right\|+r\|\stackrel{\circ}{\nabla} G\|^{2}\right) \\
& +|G|\left([G]_{\alpha}\left\|\stackrel{\circ}{B}\left(S_{k}\right)\right\|^{3}+[\stackrel{\circ}{\nabla} G]_{\alpha}\left\|\stackrel{\circ}{B}\left(S_{k}\right)\right\|^{2}+\left[\stackrel{\circ}{\nabla}^{2} G\right]_{\alpha}\left\|\stackrel{\circ}{B}\left(S_{k}\right)\right\|^{2}\right) \\
& +\|\stackrel{\circ}{\nabla} G\|\left([G]_{\alpha}\left\|\stackrel{\circ}{B}\left(S_{k}\right)\right\|^{2}+[\stackrel{\circ}{\nabla} G]_{\alpha}\left\|\stackrel{\circ}{B}\left(S_{k}\right)\right\|\right) \\
& +r\|\stackrel{\circ}{\nabla} G\|\left([\stackrel{\circ}{\nabla} G]_{\alpha}\left\|\stackrel{\circ}{\nabla}^{2} G\right\|+\|\stackrel{\circ}{\nabla} G\|\left[\stackrel{\circ}{\nabla}^{2} G\right]_{\alpha}\right) \\
& \left.+\left\|\stackrel{\circ}{\nabla}^{2} G\right\|\left([G]_{\alpha}\left\|\stackrel{\circ}{B}\left(S_{k}\right)\right\|+r[\stackrel{\circ}{\nabla} G]_{\alpha}\|\stackrel{\circ}{\nabla} G\|\right)\right) \\
& \leq \frac{C}{r}\left(|G|+r\|\stackrel{\circ}{\nabla} G\|+r^{2}\left\|\stackrel{\circ}{ }^{2} G\right\|+r^{2+\alpha}\left[\stackrel{\circ}{ }^{2} G\right]_{\alpha}\right)^{2} \\
& +C r^{3}\|\stackrel{\circ}{\nabla} G\|^{2}\left(\left\|\stackrel{\circ}{ }^{2} G\right\|+r^{\alpha}\left[\stackrel{\circ}{ }^{2} G\right]_{\alpha}\right) .
\end{aligned}
$$

The reason the estimate for $\left|\stackrel{\circ}{H}-\frac{2}{r}\right|$ is so much better is because we can expand

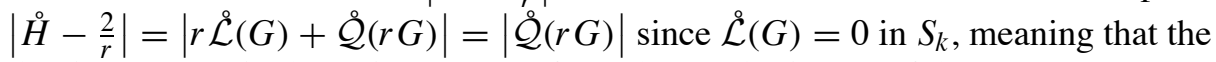
dominant terms in the estimate come from the quadratic part of the mean curvature. To proceed, we note that by standard interior elliptic estimates for $G$, we have 
$|G|+r\|\stackrel{\circ}{\nabla} G\|+r^{2}\left\|\stackrel{\circ}{\nabla}^{2} G\right\|+r^{2+\alpha}\left[\stackrel{\circ}{\nabla}^{2} G\right]_{\alpha} \leq C \varepsilon$ for some constant $C$ independent of $r$ or $\varepsilon$. Therefore

$$
\|\stackrel{\circ}{B}\|+r^{\alpha}[\stackrel{\circ}{B}]_{\alpha} \leq \frac{C}{r} \quad \text { and } \quad\left|\stackrel{\circ}{H}-\frac{2}{r}\right|+r^{\alpha}[\stackrel{\circ}{H}]_{\alpha} \leq \frac{C \varepsilon^{2}}{r}
$$

in the spherical region $S_{k}$.

Step 2. The next step is to look inside one of the scaled normal coordinate charts used in the definition of the necks. Again, the calculations are performed with respect to the scaled Euclidean metric, and computed in the transition region $T_{k}:=$ $\tilde{N}_{k} \cap\left\{(t, x): x \in B_{\varepsilon_{k}^{3 / 4}}(0) \backslash B_{\varepsilon_{k}^{3 / 4} / 2}(0)\right\}$ as well as the neck region $N_{k}:=\tilde{N}_{k} \cap$ $\left\{(t, x): x \in B_{\varepsilon_{k}^{3 / 4} / 2}(0)\right\}$ itself. Of course, the estimates in $N_{k}$ are extremely straightforward since $N_{k}$ is exactly the standard catenoid for which $\stackrel{\circ}{=} 0$ and $\|\stackrel{\circ}{B}\|=\sqrt{2} \varepsilon_{k}\|x\|^{-2}$. Hence the challenge lies in estimating $\stackrel{\circ}{H}$ and $\stackrel{\circ}{B}$ in $T_{k}$.

The transition region $T_{k}$ is the graph of the function $\tilde{F}:=\eta F+(1-\eta) G$ as in (3.3), where $F(x):=\varepsilon_{k}\left(\operatorname{arccosh}\left(\|x\| / \varepsilon_{k}\right)+d_{k}+\delta_{k}\right)$ and $G$ has an asymptotic expansion that matches the asymptotic expansion of $F$ except for the mismatch introduced by $\delta_{k}$, while $\eta$ is a cut-off whose derivative is supported in $\frac{1}{2} \varepsilon_{k}^{3 / 4} \leq\|x\| \leq$ $\varepsilon_{k}^{3 / 4}$. Now, the scaled Euclidean second fundamental form and mean curvature of a graph are

$$
\begin{aligned}
\stackrel{\circ}{B}_{i j} & =\frac{1}{\stackrel{\circ}{D}} \stackrel{\circ}{\nabla}_{i j}^{2} \tilde{F} \\
\stackrel{\circ}{H} & =\frac{1}{\stackrel{\circ}{D}}\left(\delta^{i j}-\frac{\stackrel{\circ}{\nabla}^{i} \tilde{F} \stackrel{\circ}{\nabla}^{j} \tilde{F}}{\stackrel{\circ}{D}^{2}}\right) \stackrel{\circ}{\nabla}_{i j}^{2} \tilde{F}
\end{aligned}
$$

where $\stackrel{\circ}{D}:=\left(1+\|\stackrel{\circ}{\nabla} \tilde{F}\|^{2}\right)^{1 / 2}$. Write $\tilde{F}:=u+F$ where $u:=(1-\eta)(G-F)$ and observe that

$$
u=\varepsilon_{k} \delta_{k}(1-\eta)+(1-\eta)(\hat{G}-\hat{F})
$$

for functions $\hat{G}$ and $\hat{F}$ of size $\mathcal{O}\left(\|x\|^{2}\right)+\mathcal{O}\left(\varepsilon^{3}\|x\|^{-2}\right)$. When $\|x\|=\mathcal{O}\left(\varepsilon^{3 / 4}\right)$ one has

$$
\begin{gathered}
\left|u-\varepsilon_{k} \delta_{k}(1-\eta)\right|+\sum_{k=1}^{3} \varepsilon^{3 \ell / 4}\left\|\stackrel{\circ}{\nabla}^{\ell} u+\varepsilon_{k} \delta_{k} \stackrel{\circ}{\nabla}^{\ell} \eta\right\|=\mathcal{O}\left(\varepsilon^{3 / 2}\right) \\
\left|F-c_{1}-c_{2} \log (1 / \varepsilon)\right|+\sum_{\ell=1}^{3} \varepsilon^{3 \ell / 4}\left\|\stackrel{\circ}{\nabla}^{\ell} F\right\|=\mathcal{O}(\varepsilon)
\end{gathered}
$$

for constants $c_{1}, c_{2}$. Thus plugging $\tilde{F}$ into the expression for $\stackrel{\circ}{B}$ and estimating yields

$$
\|\stackrel{\circ}{B}\|+\varepsilon^{3 \alpha / 4}[\stackrel{\circ}{B}]_{\alpha} \leq C \varepsilon^{-1 / 2} .
$$


Next, plugging $\tilde{F}$ into the expression for $\stackrel{\circ}{H}$ yields

$$
\begin{aligned}
\stackrel{\circ}{D} \stackrel{\circ}{H}=\stackrel{\circ}{\Delta} & +\left(\frac{1}{\sqrt{1+\|\stackrel{\circ}{\nabla} F\|^{2}}}-\frac{1}{\sqrt{1+\|\stackrel{\circ}{\nabla} F+\stackrel{\circ}{\nabla} u\|^{2}}}\right) F_{, i} F_{, j} F_{, i j} \\
& -\frac{u, i u_{, j} u_{, i j}+u_{, i} u_{, j} F_{, i j}+2 u_{, i} F_{, j} u_{, i j}+F_{, i} F_{, j} u_{, i j}+2 F_{, i} u, j F_{, i j}}{\sqrt{1+\|\stackrel{\circ}{\nabla} F+\stackrel{\circ}{\nabla} u\|^{2}}}
\end{aligned}
$$

where we have used $\stackrel{\circ}{\Delta} F-\left(1+\|\stackrel{\circ}{\nabla} F\|^{2}\right)^{-1} F_{, i} F_{, j} F_{, i j} \equiv 0$. This holds since $F$ is the graphing function for the catenoid which has zero mean curvature. Therefore after some work one can estimate

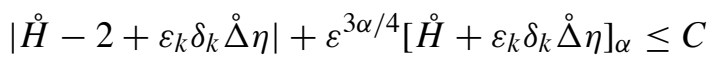

where $C$ is independent of $\varepsilon$. We remind the reader that the estimates derived here are with respect to the $r$-scaled Euclidean metric in the coordinate chart. When we make use of these estimates in the next two steps, we will need to reverse the scaling.

Step 3. The next step is to compute the pointwise norm of $H:=H\left[\tilde{\Sigma}_{r}^{F}(\sigma, \delta)\right]$ with respect to the actual background metric with the help of the expansions from Lemma 2.3. This lemma can be invoked here because we have also shown in Steps 1 and 2 that $\|Y\|\|\stackrel{\circ}{B}\|+\|Y\|^{1+\alpha}[\stackrel{\circ}{B}]_{\alpha} \leq C$ everywhere on $\tilde{\Sigma}_{r}^{F}(\sigma, \delta)$.

To proceed, we again perform the necessary calculations in each of the different regions identified in Steps 1 and 2. Consider first the spherical region $S_{k}$, where $\|Y\|=\mathcal{O}(r)$ and

$$
\begin{aligned}
\left|H-\frac{2}{r}\right|+r^{\alpha}[H]_{\alpha} \leq & \left|\stackrel{\circ}{H}-\frac{2}{r}\right|+r^{\alpha}[\stackrel{\circ}{H}]_{\alpha}+\left|\mathcal{H}^{(00)}(Y, \stackrel{\circ}{B})\right| \\
& +r^{\alpha}\left[\mathcal{H}^{(00)}(Y, \stackrel{\circ}{B})\right]_{\alpha}+C\|Y\|^{3} \\
\leq & \left|\stackrel{\circ}{H}-\frac{2}{r}\right|+r^{\alpha}[\stackrel{\circ}{H}]_{\alpha}+C\|Y\|+C\|Y\|^{2}\|\stackrel{\circ}{B}\| \\
\leq & C\left(\frac{\varepsilon^{2}}{r}+r\right)
\end{aligned}
$$

by previous estimates and inspection of the quantity $\mathcal{H}^{(00)}(Y, B)$ given in Lemma 2.3. Consider now the transition region $T_{k}$. First, by reversing the scaling used in Step 2, the second fundamental form and mean curvature in $\tilde{N}_{k}$, measured with respect to the Euclidean metric, satisfy the estimates

$$
\left|\stackrel{\circ}{H}-\frac{2}{r}+\varepsilon_{k} \delta_{k} \stackrel{\circ}{\Delta}\right|+r^{\alpha} \varepsilon^{3 \alpha / 4}\left[\stackrel{\circ}{H}+\varepsilon_{k} \delta_{k} \stackrel{\circ}{\Delta} \eta\right]_{\alpha} \leq \frac{C}{r} \text { and }\|\stackrel{\circ}{B}\|+r^{\alpha} \varepsilon^{3 \alpha / 4}[\stackrel{\circ}{B}]_{\alpha} \leq \frac{C}{r \varepsilon^{1 / 2}} .
$$


Now these estimates can be plugged into the expansion of the mean curvature, except that $Y$ is now the position vector field of $\psi^{-1}\left(\tilde{N}_{k}\right)$ relative to the center of the normal coordinate chart used in the construction of the neck. Thus $\|Y\|=\mathcal{O}\left(r \varepsilon^{3 / 4}\right)$ so that

$$
\left|H-\frac{2}{r}\right|+r^{\alpha} \varepsilon^{3 \alpha / 4}[H]_{\alpha} \leq \frac{C}{r}\left(1+\frac{\delta}{r \varepsilon^{1 / 2}}\right)
$$

in the transition region. Finally, in order to obtain the required estimate in the neck region itself, we must use the relation $\|Y\|^{2}=r^{2}\left(\|x\|^{2}+\varepsilon^{2} \operatorname{arccosh}^{2}(\|x\| / \varepsilon)\right)$ between the position vector field and the $x$-coordinate used in the definition of the neck. This allows us to deduce

$$
\left|H-\frac{2}{r}\right|+\|Y\|^{\alpha}[H]_{\alpha} \leq \frac{2}{r}+C r\|x\|+C r^{2}\|x\|^{2} \cdot \frac{\sqrt{2} \varepsilon_{k}}{r\|x\|^{2}} \leq \frac{C}{r}+C r(\|x\|+\varepsilon) \text {. }
$$

Step 4. The remaining task is to estimate $\left|H-\frac{2}{r}\right|$ in the $C_{\nu-2}^{0, \alpha}$ norm for $v \in$ $(1,2)$. This estimate will be derived by once again considering the three regions of $\tilde{\Sigma}_{r}^{F}(\sigma, \delta)$ identified in the previous steps. For now, the only assumption that will be made about $\varepsilon$ and $\delta$ is that $\varepsilon \ll r$. First, consider a spherical region $S_{k}$ where $\zeta_{r} \equiv r$. Hence

$$
\zeta_{r}^{2-v}\left|H-\frac{2}{r}\right|+\zeta_{r}^{2+\alpha-v}\left[H-\frac{2}{r}\right]_{\alpha} \leq C r^{3-v} .
$$

Next, in the transition region $T_{k}$ where $\zeta_{r}=\mathcal{O}\left(r \varepsilon^{3 / 4}\right)$ one has

$$
\zeta_{r}^{2-v}\left|H-\frac{2}{r}\right|+\zeta_{r}^{2+\alpha-v}\left[H-\frac{2}{r}\right]_{\alpha} \leq C r^{1-v}\left(\varepsilon^{3(2-v) / 4}+\delta \varepsilon^{1-3 v / 4}\right) .
$$

Finally, in the neck region $\tilde{N}_{k}$ where $\zeta_{r}(x)=r\|x\|$ in the local coordinates used there one has

$$
\zeta_{r}^{2-v}\left|H-\frac{2}{r}\right|+\zeta_{r}^{2+\alpha-v}\left[H-\frac{2}{r}\right]_{\alpha} \leq C r^{1-v} \varepsilon^{3(2-v) / 4} .
$$

The proposition now follows by consolidating these three estimates.

As a by-product of the proof of the previous proposition, we have also obtained a uniform bound for the weighted norm of the second fundamental form in the geodesic normal coordinate charts used in the construction of $\tilde{\Sigma}_{r}^{F}(\sigma, \delta)$.

Corollary 5.4. The second fundamental form of $\tilde{\Sigma}_{r}^{F}(\sigma, \delta)$ with respect to the Euclidean metric in any geodesic normal coordinate chart $\mathcal{U}$ used in the construction of $\tilde{\Sigma}_{r}^{F}(\sigma, \delta)$ satisfies the estimate

$$
\sup _{\mathcal{U}}\left(\zeta_{r}|\stackrel{\circ}{B}|+\zeta_{r}^{2}\|\stackrel{\circ}{\nabla} \stackrel{\circ}{B}\|\right)+\zeta_{r}^{2+\alpha}[\stackrel{\circ}{\nabla} \stackrel{\circ}{B}]_{\alpha, \mathcal{U}} \leq C
$$

for some constant $C$ independent of $r, \varepsilon, \delta$ and $K$. 
Proof. The proof of Proposition 5.3 has already established that $\zeta_{r}\|\stackrel{B}{\|}\|+\zeta_{r}^{1+\alpha}[\stackrel{\circ}{B}]_{\alpha} \leq$ $C$ for some constant $C$ independent of $r, \varepsilon, \delta$ and $K$ in every normal coordinate chart used in the definition of $\tilde{\Sigma}_{r}^{F}(\sigma, \delta)$. It thus remains to estimate $\left\|\stackrel{\circ}{\nabla}^{B}\right\|$.

First, consider one of the spherical regions of $\tilde{\Sigma}_{r}^{F}(\sigma, \delta)$. The calculations from Section 2.2 when $\Sigma$ is a sphere of radius $r$ and $f=r G$ where $G$ is the function from equation (3.1) give

$$
[\stackrel{\circ}{B}(r G)]_{i j}=\frac{(1-G)^{2} \stackrel{\circ}{h}_{i j}+r^{2}(1-G) G_{; i j}+r^{2} G_{; i} G_{; j}}{r\left((1-G)^{2}+r^{2}\|\stackrel{\circ}{\nabla} G\|^{2}\right)^{1 / 2}}
$$

for the second fundamental form $\stackrel{\circ}{B}(r G)$ of $\tilde{\Sigma}_{r}(\sigma, \delta)$ with respect to the Euclidean metric while $\grave{h}_{i j}$ is the induced metric of the sphere of radius $r$. Similarly, the induced metric $[\stackrel{\circ}{h}(r G)]_{i j}$ and Christoffel symbols $\left.\left[\stackrel{\circ}{\Gamma}^{(r} G\right)\right]_{i j k}$ of $\tilde{\Sigma}_{r}^{F}(\sigma, \delta)$ with respect to the Euclidean metric of the coordinate chart are related to the corresponding quantities of the sphere of radius $r$ by

$[\stackrel{\circ}{h}(r G)]_{i j}=(1-G)^{2} \stackrel{\circ}{h}_{i j}+r^{2} G_{; i} G_{; j} \quad$ and $\quad[\stackrel{\circ}{\Gamma}(r G)]_{i j k}=(1-G)^{2} \stackrel{\circ}{\Gamma}_{i j k}+\mathcal{G}_{1}+r^{2} \mathcal{G}_{2}$

where $\mathcal{G}_{1}$ is a tensor formed out of $\mathcal{O}(1)$ linear combinations of components of $\stackrel{\circ}{\nabla} G \otimes \stackrel{h}{h}$ and $\mathcal{G}_{2}$ is a tensor formed out of $\mathcal{O}(1)$ linear combinations of components of $\stackrel{\circ}{\nabla}^{2} G \otimes \stackrel{\circ}{\nabla} G$. We now compute

$$
\begin{aligned}
{[\stackrel{\circ}{B}(r G)]_{i j ; k}=} & {[\stackrel{\circ}{B}(r G)]_{i j, k}-[\stackrel{\circ}{B}(r G)]_{s j}[\stackrel{\circ}{h}(r G)]^{s t}[\stackrel{\circ}{\Gamma}(r G)]_{i k t} } \\
& -[\stackrel{\circ}{B}(r G)]_{i s}[\stackrel{\circ}{h}(r G)]^{s t}[\stackrel{\circ}{\Gamma}(r G)]_{j k t}
\end{aligned}
$$

and after some work, we reach the desired estimate. The Hölder seminorm estimate

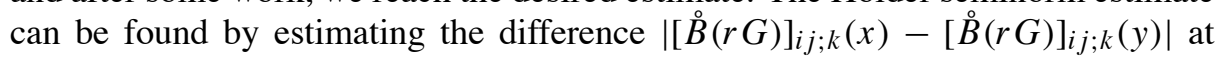
two different points $x, y$ using the above formula. A similar procedure, based on differentiating the formula for the second fundamental form of a graph in Euclidean space found in Step 2 of the proof of Proposition 5.3, yields the desired estimate for $\|\stackrel{\nabla}{ } \stackrel{B}{\|}\|$ in the neck and transition regions. The details are left to the reader.

\subsubsection{The operator estimates}

The next task is to find estimates for the $C_{v}^{0, \alpha}$ norm of the quadratic remainder term $\mathcal{Q}$ and the error term $\mathcal{H}$ for the finite-length surface. We do this by combining the estimates from Section 2 with the specifics of the construction of $\tilde{\Sigma}_{r}^{F}(\sigma, \delta)$ from Section 3. But we first need to show that $|f|\|\stackrel{B}{\|}\|+\|\stackrel{\circ}{\nabla}\| \ll 1$ holds if $|f|_{C_{v}^{2, \alpha}}$ is bounded and $r$ is small enough.

Lemma 5.5. Let $\mathcal{U} \subseteq \tilde{\Sigma}_{r}^{F}(\sigma, \delta)$ be one of the normal coordinate charts used in the construction of $\tilde{\Sigma}_{r}^{F}(\sigma, \delta)$ where the second fundamental form with respect to the Euclidean metric is $\stackrel{\bullet}{B}$. Then the following estimate hold in $\mathcal{U}$ :

$$
\sup _{x \in \mathcal{U}}\left(\|Y(x)\|\|\stackrel{\circ}{B}(x)\|+\|Y(x)\|^{1+\alpha}[\stackrel{\circ}{B}]_{\alpha}\right) \leq C
$$


and

$$
\begin{gathered}
\sup _{x \in \mathcal{U}}(|f(x)|\|\stackrel{\circ}{B}(x)\|+\|\stackrel{\circ}{\nabla} f(x)\|)+\sup _{x \in \mathcal{U}} \zeta_{r}^{\alpha}(x)[f \cdot \stackrel{\circ}{B}+\stackrel{\circ}{\nabla} f]_{\alpha, \mathcal{U}} \\
\leq C r^{\nu-1}|f|_{C_{v}^{2, \alpha}}
\end{gathered}
$$

for all $f \in C_{*}^{2, \alpha}\left(\tilde{\Sigma}_{r}^{F}(\sigma, \delta)\right)$. Here $C$ is a constant independent of $r, \varepsilon$ and $\delta$.

Proof. The first estimate is proved in Steps 1 and 2 of Proposition 5.3. For the second estimate, we not that Corollary 5.4 implies that we have $\zeta_{r}(x)\|\stackrel{\circ}{B}(x)\|+$ $\zeta_{r}^{2}(x)\|\stackrel{\circ}{\nabla} \stackrel{\circ}{B}(x)\| \leq C$ where $C$ is some constant independent of $r$, no matter where $x$ is located in $\tilde{\Sigma}_{r}^{F}(\sigma, \delta)$. Consequently,

$$
\begin{gathered}
|f(x)|\|\stackrel{\circ}{B}(x)\|+\|\stackrel{\circ}{\nabla} f(x)\|=\zeta_{r}^{\nu-1}(x)\left(\zeta_{r}^{-v}(x)|f(x)| \cdot \zeta_{r}(x)\|\stackrel{\circ}{B}(x)\|\right. \\
\left.+\zeta_{r}^{1-v}(x)\|\stackrel{\circ}{\nabla} f(x)\|\right)
\end{gathered}
$$

which yields the first of the desired estimates. For the other, we calculate

$$
\begin{aligned}
\sup _{\mathcal{U}} \zeta_{r}^{\alpha} \cdot[ & f \cdot \stackrel{\circ}{B}+\stackrel{\circ}{\nabla} f]_{\alpha, \mathcal{U}} \leq \sup _{\mathcal{U}} \zeta_{r}^{\alpha}\left([f]_{\alpha, U}\|\stackrel{\circ}{B}\|+|f|[\stackrel{\circ}{B}]_{\alpha}+[\stackrel{\circ}{\nabla} f]_{\alpha}\right) \\
& \leq \sup _{\mathcal{U}} \zeta_{r}^{\nu-1}\left(\zeta_{r}^{\alpha-v}[f]_{\alpha} \zeta_{r}\|\stackrel{\circ}{B}\|+\zeta_{r}^{-v}|f| \zeta_{r}^{1+\alpha}[\stackrel{\circ}{B}]_{\alpha, \mathcal{U}}+\zeta_{r}^{1+\alpha-v}[\stackrel{\circ}{\nabla} f]_{\alpha, \mathcal{U}}\right) \\
& \leq C r^{\nu-1}|f|_{C_{v}^{2, \alpha}}
\end{aligned}
$$

by the Hölder seminorm estimate of Corollary 5.4 .

We can now deduce the following.

Proposition 5.6. There exists $M>0$ so that if $f_{1}, f_{2} \in C_{v}^{2, \alpha}\left(\tilde{\Sigma}_{r}^{F}(\sigma, \delta)\right)$ for $v \in$

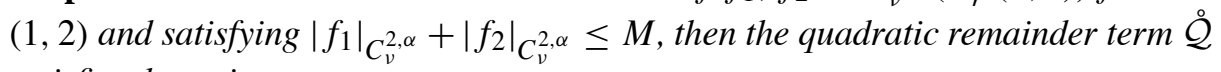
satisfies the estimate

$$
\left|\stackrel{\mathcal{Q}}{(}\left(f_{1}\right)-\stackrel{\mathcal{Q}}{(}\left(f_{2}\right)\right|_{C_{\nu-2}^{0, \alpha}} \leq C r^{\nu-1}\left|f_{1}-f_{2}\right|_{C_{v}^{2, \alpha}} \max \left\{\left|f_{1}\right|_{C_{v}^{2, \alpha}},\left|f_{2}\right|_{C_{v}^{2, \alpha}}\right\}
$$

where $C$ is a constant independent of $r, \varepsilon$ and $\delta$.

Proof. Since Lemma 5.5 ensures that $\left|f_{i}\right|\|\stackrel{\circ}{B}\|+\left\|\stackrel{\circ}{\nabla} f_{i}\right\|$ can be made as small as 
needed, it is possible to invoke Lemma 2.5 and obtain

$$
\begin{aligned}
& \zeta_{r}^{2-v}\left|\mathcal{Q}\left(f_{1}\right)-\mathcal{Q}\left(f_{2}\right)\right| \\
& \leq C \zeta_{r}^{-v}\left|f_{1}-f_{2}\right| \\
& \cdot \max _{i} \zeta_{r}^{2}\left(\left|f_{i}\right|\|\stackrel{\circ}{B}\|^{3}+\left\|\stackrel{\circ}{\nabla} f_{i}\right\|\|\stackrel{\circ}{B}\|^{2}+\left\|\stackrel{\circ}{\nabla} f_{i}\right\|\|\stackrel{\circ}{\nabla} \stackrel{\circ}{B}\|+\left\|\stackrel{\circ}{\nabla^{2}} f_{i}\right\|\|\stackrel{\circ}{B}\|\right) \\
& +C \zeta_{r}^{1-v}\left\|\stackrel{\circ}{\nabla} f_{1}-\stackrel{\circ}{\nabla} f_{2}\right\| \\
& \text { - } \max _{i} \zeta_{r}\left(\left|f_{i}\right|\|\stackrel{\circ}{B}\|^{2}+\left\|\stackrel{\circ}{\nabla} f_{i}\right\|\|\stackrel{\circ}{B}\|+\left|f_{i}\right|\|\stackrel{\circ}{\nabla} \stackrel{\circ}{B}\|+\left\|\stackrel{\circ}{\nabla} f_{i}\right\|\left\|\stackrel{\circ}{\nabla}^{2} f_{i}\right\|\right) \\
& +C \zeta_{r}^{2-v}\left\|\stackrel{\circ}{\nabla}^{2} f_{1}-\stackrel{\circ}{\nabla}^{2} f_{2}\right\| \cdot \max _{i}\left(\left|f_{i}\right|\|\stackrel{\circ}{B}\|+\left\|\stackrel{\circ}{\nabla} f_{i}\right\|^{2}\right) \\
& \leq C\left|f_{1}-f_{2}\right|_{C_{v}^{2, \alpha}} \\
& \cdot \max _{i}\left\{\left|f_{i}\right|_{C_{\nu}^{2, \alpha}}\right\}\left(\sum_{k=1}^{3}\|\stackrel{\circ}{B}\|^{k} \zeta^{k-1+v}+\| \stackrel{\circ}{\nabla} \stackrel{\circ}{\|} \zeta_{r}^{1+v}+\left|f_{i}\right|_{C_{\nu}^{2, \alpha}} \zeta_{r}^{2(\nu-1)}+\zeta_{r}^{\nu-1}\right)
\end{aligned}
$$

as well as

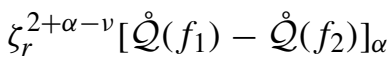

$$
\begin{aligned}
& \leq C \zeta_{r}^{\alpha-v}\left[f_{1}-f_{2}\right]_{\alpha} \\
& \cdot \max _{i} \zeta_{r}^{2}\left(\left|f_{i}\right|\|\stackrel{\circ}{B}\|^{3}+\left\|\stackrel{\circ}{\nabla} f_{i}\right\|\|\stackrel{\circ}{B}\|^{2}+\left\|\stackrel{\circ}{\nabla} f_{i}\right\|\|\stackrel{\circ}{\nabla} \stackrel{\circ}{B}\|+\left\|\stackrel{\circ}{\nabla}^{2} f_{i}\right\|\|\stackrel{\circ}{B}\|\right) \\
& +C \zeta_{r}^{1+\alpha-v}\left[\stackrel{\circ}{\nabla} f_{1}-\stackrel{\circ}{\nabla} f_{2}\right]_{\alpha} \\
& \cdot \max _{i} \zeta_{r}\left(\left|f_{i}\right|\|\stackrel{\circ}{B}\|^{2}+\left\|\stackrel{\circ}{\nabla} f_{i}\right\|\|\stackrel{\circ}{B}\|+\left|f_{i}\right|\|\stackrel{\circ}{\nabla} \stackrel{\circ}{B}\|+\left\|\stackrel{\circ}{\nabla} f_{i}\right\|\left\|\stackrel{\circ}{\nabla}^{2} f_{i}\right\|\right) \\
& +C \zeta_{r}^{2+\alpha-v}\left[\stackrel{\circ}{\nabla}^{2} f_{1}-\stackrel{\circ}{\nabla}^{2} f_{2}\right]_{\alpha} \cdot \max _{i}\left(\left|f_{i}\right|\|\stackrel{\circ}{B}\|+\left\|\stackrel{\circ}{\nabla} f_{i}\right\|^{2}\right) \\
& +C \zeta_{r}^{-v}\left|f_{1}-f_{2}\right| \cdot \zeta_{r}^{2+\alpha} \max _{i}\left(\left[f_{i}\right]_{\alpha}\|\stackrel{\circ}{B}\|^{3}+\left|f_{i}\right|\|\stackrel{\circ}{B}\|^{2}[\stackrel{\circ}{B}]_{\alpha}+\left[\stackrel{\circ}{\nabla} f_{i}\right]_{\alpha}\|\stackrel{\circ}{B}\|^{2}\right. \\
& \left.+\left\|\stackrel{\circ}{\nabla} f_{i}\right\|\|\stackrel{\circ}{B}\|[\stackrel{\circ}{B}]_{\alpha}+\left[\stackrel{\circ}{\nabla} f_{i}\right]_{\alpha}\|\stackrel{\circ}{\nabla} \stackrel{\circ}{B}\|+\left\|\stackrel{\circ}{\nabla} f_{i}\right\|[\stackrel{\circ}{\nabla} \stackrel{\circ}{B}]_{\alpha}+\left[\stackrel{\circ}{\nabla}^{2} f_{i}\right]_{\alpha}\|\stackrel{\circ}{B}\|+\left\|\nabla^{2} f_{i}\right\|[\stackrel{\circ}{B}]_{\alpha}\right) \\
& +C \zeta_{r}^{1-v}\left\|\stackrel{\circ}{\nabla} f_{1}-\stackrel{\circ}{\nabla} f_{2}\right\| \cdot \max _{i} \zeta_{r}^{1+\alpha}\left(\left[f_{i}\right]_{\alpha}\|\stackrel{\circ}{B}\|^{2}+\left|f_{i}\right|\|\stackrel{\circ}{B}\|[\stackrel{\circ}{B}]_{\alpha}+\left[\stackrel{\circ}{\nabla} f_{i}\right]_{\alpha}\|\stackrel{\circ}{B}\|\right. \\
& \left.+\left\|\stackrel{\circ}{\nabla} f_{i}\right\|[\stackrel{\circ}{B}]_{\alpha}+\left[f_{i}\right]_{\alpha}\|\stackrel{\circ}{\nabla} \stackrel{\circ}{B}\|+\left|f_{i}\right|[\stackrel{\circ}{\nabla} \stackrel{\circ}{B}]_{\alpha}+\left[\stackrel{\circ}{\nabla} f_{i}\right]_{\alpha}\left\|\stackrel{\circ}{\nabla}^{2} f_{i}\right\|+\left\|\stackrel{\circ}{\nabla} f_{i}\right\|\left[\stackrel{\circ}{\nabla}^{2} f_{i}\right]_{\alpha}\right) \\
& +C \zeta_{r}^{2-v}\left\|\stackrel{\circ}{\nabla}^{2} f_{1}-\stackrel{\circ}{\nabla}^{2} f_{2}\right\| \cdot \max _{i} \zeta_{r}^{\alpha}\left(\left[f_{i}\right]_{\alpha}\|\stackrel{\circ}{B}\|+\left|f_{i}\right|[\stackrel{\circ}{B}]_{\alpha}+\left[\stackrel{\circ}{\nabla} f_{i}\right]_{\alpha}\left\|\stackrel{\circ}{\nabla} f_{i}\right\|\right) \\
& \leq C\left|f_{1}-f_{2}\right|_{C_{v}^{2, \alpha}} \\
& \cdot \max _{i}\left\{\left|f_{i}\right|_{C_{v}^{2, \alpha}}\right\}\left(\sum_{k=1}^{3}\|\stackrel{\circ}{B}\|^{k} \zeta^{k-1+v}+\|\stackrel{\circ}{\nabla} \stackrel{\circ}{B}\| \zeta_{r}^{1+v}+\left|f_{i}\right|_{C_{v}^{2, \alpha}} \zeta_{r}^{2(\nu-1)}\right. \\
& \left.+\sum_{k=0}^{2}\|\stackrel{\circ}{B}\|^{k}[\stackrel{\circ}{B}]_{\alpha} \zeta_{r}^{k+\alpha+v}+[\stackrel{\circ}{\nabla} \stackrel{\circ}{B}]_{\alpha} \zeta_{r}^{1+\alpha+v}+\zeta_{r}^{\nu-1}\right) .
\end{aligned}
$$


The desired estimate now follows by Corollary 5.4.

Proposition 5.7. There exists $M>0$ so that if $f_{1}, f_{2} \in C_{v}^{2, \alpha}\left(\tilde{\Sigma}_{r}^{F}(\sigma, \delta)\right)$ for $v \in$ $(1,2)$ and satisfying $\left|f_{1}\right|_{C_{v}^{2, \alpha}}+\left|f_{2}\right|_{C_{v}^{2, \alpha}} \leq M$, then

$$
\begin{aligned}
& \left|\mathcal{H}^{(1)}\left(f_{1}\right)-\mathcal{H}^{(1)}\left(f_{2}\right)\right|_{C_{\nu-2}^{0, \alpha}} \\
& \quad \leq C r^{2}\left(1+r^{\nu-1} \max \left\{\left|f_{1}\right|_{\left.\left.C_{\nu}^{2, \alpha},\left|f_{2}\right|_{C_{v}^{2, \alpha}}\right\}\right)\left|f_{1}-f_{2}\right|_{C_{\nu}^{2, \alpha}}}\right.\right.
\end{aligned}
$$

where $C$ is a constant independent of $r, \varepsilon$ and $\delta$.

Proof. First observe that $\left\|Y_{f}\right\| \leq C(r+|f|) \leq C\left(r+r^{\nu}|f|_{C_{v}^{2, \alpha}}\right) \leq C r$ by the uniform boundedness of $|f|_{C_{v}^{2, \alpha}}$ and the fact that $v \in(1,2)$. Similarly, it is also true that $\zeta_{r}^{\alpha}\left[Y_{f}\right]_{\alpha} \leq C\left(r+\zeta_{r}^{\alpha}[f]_{\alpha}+|f| \zeta_{r}^{\alpha}[\stackrel{\circ}{N}]_{\alpha}\right) \leq C r$ since we can show that the $\alpha$-Hölder norm of the unit normal vector field $\stackrel{\circ}{N}$ with respect to the Euclidean metric is uniformly bounded. This is turn follows because $\left\|\nabla^{\circ} \stackrel{\circ}{N}\right\| \leq C\|\stackrel{\circ}{B}\|$ and we have a bound for $\zeta_{r}\|\stackrel{\circ}{B}\|$ from Lemma 5.5. Next, since $\left|f_{i}\right|\|\stackrel{\circ}{ }\|+\left\|\stackrel{\circ}{\nabla} f_{i}\right\|$ can be made as small as needed according to Lemma 5.5, we can use Proposition 2.6 and Corollary 5.4 to derive

$$
\begin{aligned}
& \zeta_{r}^{2-v}\left|\mathcal{H}^{(1)}\left(f_{1}\right)-\mathcal{H}^{(1)}\left(f_{2}\right)\right| \\
& \leq C \zeta_{r}^{2-v}\left\|\stackrel{\circ}{B}_{f_{1}}-\stackrel{\circ}{B}_{f_{2}}\right\| \max _{i}\left\|Y_{f_{i}}\right\|^{2} \\
& +C \max _{i} \zeta_{r}\left\|\stackrel{\circ}{B}_{f_{i}}\right\|\left(\zeta_{r}\left\|Y_{f_{i}}\right\|\left(1+\left\|Y_{f_{i}}\right\|\|\stackrel{\circ}{B}\|\right) \zeta_{r}^{-v}\left|f_{1}-f_{2}\right|\right. \\
& \left.+\left\|Y_{f_{i}}\right\|^{2} \zeta_{r}^{1-v}\left\|\stackrel{\circ}{\nabla} f_{1}-\stackrel{\circ}{\nabla} f_{2}\right\|\right) \\
& \quad+C \max _{i}\left(\zeta_{r}^{2-v}\left|f_{1}-f_{2}\right|+\zeta_{r}\left\|Y_{f_{i}}\right\|\left(\zeta_{r}^{-v}\left|f_{1}-f_{2}\right| \zeta_{r}\|\stackrel{\circ}{\nabla}\|\right.\right. \\
& \left.\left.f_{1}-\stackrel{\circ}{\nabla} f_{2} \|\right)\right) \\
& \leq C r^{2} \zeta_{r}^{2-v}\left\|\stackrel{\circ}{B}_{f_{1}}-\stackrel{\circ}{B}_{f_{2}}\right\|+C r^{2}\left|f_{1}-f_{2}\right| C_{v}^{2, \alpha} \\
& \leq C r^{2}\left(1+r^{\nu-1} \max \left\{\left|f_{1}\right|_{C_{v}^{2, \alpha}},\left|f_{2}\right|_{\left.\left.C_{v}^{2, \alpha}\right\}\right)\left|f_{1}-f_{2}\right|_{C_{v}^{2, \alpha}}}\right.\right.
\end{aligned}
$$

where to reach the last line above, we have used the pointwise bound for $\| \stackrel{\circ}{B}_{f_{1}}$ $\stackrel{\circ}{B}_{f_{2}} \|$ from Lemma 2.5 and the techniques used in Proposition 5.6 to estimate its $C_{v-2}^{0, \alpha}$ norm. Finally, we perform similar calculations on the Hölder seminorm esti- 
mate of Proposition 2.6 to deduce

$$
\begin{aligned}
& \zeta_{r}^{2+\alpha-v}\left[\mathcal{H}^{(1)}\left(f_{1}\right)-\mathcal{H}^{(1)}\left(f_{2}\right)\right]_{\alpha} \\
& \leq C r \zeta_{r}^{2+v}\left\|\stackrel{\circ}{B}_{f_{1}}-\stackrel{\circ}{B}_{f_{2}}\right\| \max _{i} \zeta_{r}^{\alpha}\left(\left[Y_{f_{i}}\right]_{\alpha}+\left\|Y_{f_{i}}\right\|\left(\left[f_{i}\right]_{\alpha}\|\stackrel{\circ}{B}\|\right.\right. \\
& \left.\left.+\left|f_{i}\right|[\stackrel{\circ}{B}]_{\alpha}+[\stackrel{\circ}{\nabla} f]_{\alpha}\right)\right) \\
& +C r^{2} \zeta_{r}^{2+\alpha-v}\left[\stackrel{\circ}{B}_{f_{1}}-\stackrel{\circ}{B}_{f_{2}}\right]_{\alpha} \\
& +C \max _{i} \zeta_{r}^{1+\alpha}\left[B_{f_{i}}\right]_{\alpha}\left(r \zeta_{r}\left(1+\left\|Y_{f_{i}}\right\|\|\stackrel{\circ}{B}\|\right) \zeta_{r}^{-v}\left|f_{1}-f_{2}\right|\right. \\
& \left.\left.+r^{2} \zeta_{r}^{1-v}\left\|\stackrel{\circ}{\nabla} f_{1}-\stackrel{\circ}{\nabla} f_{2}\right\|\right)\right) \\
& +C \zeta_{r} \max _{i}\left(\zeta_{r}^{1+\alpha}\left(\left[f_{i}\right]_{\alpha}\|\stackrel{\circ}{B}\|+\left|f_{i}\right|[\stackrel{\circ}{B}]_{\alpha}+\left[\stackrel{\circ}{\nabla} f_{i}\right]_{\alpha}\right) \zeta_{r}^{-v}\left|f_{1}-f_{2}\right|\right. \\
& +\zeta_{r}^{\alpha-v}\left[f_{1}-f_{2}\right]_{\alpha} \\
& +\zeta_{r}^{1+\alpha}\left(\left[Y_{f_{i}}\right]_{\alpha}+\left\|Y_{f_{i}}\right\|\left(\left[f_{i}\right]_{\alpha}\|\stackrel{\circ}{B}\|+\left|f_{i}\right|[\stackrel{\circ}{B}]_{\alpha}+\left[\stackrel{\circ}{\nabla} f_{i}\right]_{\alpha}\right)\right) \\
& \times\left(\zeta_{r}^{-v}\left|f_{1}-f_{2}\right| \zeta_{r}\|\stackrel{\circ}{B}\|+\zeta_{r}^{1-v}\left\|\stackrel{\circ}{\nabla} f_{1}-\stackrel{\circ}{\nabla} f_{2}\right\|\right) \\
& +r\left(\zeta_{r}^{\alpha-v}\left[f_{1}-f_{2}\right]_{\alpha} \zeta_{r}\|\stackrel{\circ}{B}\|+\zeta_{r}^{-v}\left|f_{1}-f_{2}\right| \zeta_{r}^{1+\alpha}[\stackrel{\circ}{B}]_{\alpha}\right. \\
& \left.\left.+\zeta_{r}^{1+\alpha-v}\left[\stackrel{\circ}{\nabla} f_{1}-\stackrel{\circ}{\nabla} f_{2}\right]_{\alpha}\right)\right) \\
& \leq C r^{2}\left(\zeta_{r}^{2+v}\left\|\stackrel{\circ}{B}_{f_{1}}-\stackrel{\circ}{B}_{f_{2}}\right\|+\zeta_{r}^{2+\alpha-v}\left[\stackrel{\circ}{B}_{f_{1}}-\stackrel{\circ}{B}_{f_{2}}\right]_{\alpha}+\left|f_{1}-f_{2}\right|_{C_{v}^{2, \alpha}}\right) \\
& +C r^{1+v}\left|f_{1}-f_{2}\right|_{C_{v}^{2, \alpha}} \\
& \leq C r^{2}\left(1+r^{\nu-1} \max \left\{\left|f_{1}\right|_{C_{v}^{2, \alpha}},\left|f_{2}\right|_{C_{v}^{2, \alpha}}\right\}\right)\left|f_{1}-f_{2}\right|_{C_{v}^{2, \alpha}}
\end{aligned}
$$

using Lemma 2.5 once again.

Proposition 5.8. There exists $M>0$ so that if $f \in C_{v}^{2, \alpha}\left(\tilde{\Sigma}_{r}^{F}(\sigma, \delta)\right)$ for $v \in(1,2)$ and satisfying $|f|_{C_{v}^{2, \alpha}} \leq M$, then

$$
|\stackrel{\mathcal{L}}{\circ} \Theta(f)-\mathcal{L}(f)|_{C_{v-2}^{0, \alpha}} \leq C r^{2}|f|_{C_{v}^{2, \alpha}}
$$

where $C$ is a constant independent of $r, \varepsilon$ and $\delta$.

Proof. We begin by estimating

$$
\begin{aligned}
|\stackrel{\mathcal{L}}{\circ} \Theta(f)-\mathcal{L}(f)|_{C_{\nu-2}^{0, \alpha}} & \leq|\stackrel{\circ}{\mathcal{L}}(\Theta(f)-f)|_{C_{\nu-2}^{0, \alpha}}+\mid(\stackrel{\mathcal{L}}{ }-\mathcal{L})(f)_{C_{\nu-2}^{0, \alpha}} \\
& \leq C|\Theta(f)-f|_{C_{\nu}^{2, \alpha}}+\mid(\mathcal{L}-\mathcal{L})(f)_{C_{\nu-2}^{0, \alpha}}
\end{aligned}
$$


where $C$ is the operator norm of $\mathcal{L}: C_{v}^{2, \alpha}\left(\tilde{\Sigma}_{r}(\sigma, \delta)\right) \rightarrow C_{v-2}^{0, \alpha}\left(\tilde{\Sigma}_{r}(\sigma, \delta)\right)$. This is a constant indpendent of $r, \varepsilon, \delta$ because of Lemma 5.5 and the following computation:

$$
\begin{aligned}
\zeta_{r}^{2-v}|\stackrel{\Delta}{\Delta}| & +\zeta_{r}^{-v}|f| \cdot \zeta_{r}^{2}\|\stackrel{\circ}{B}\|^{2}+\zeta_{r}^{2+\alpha-v}\left[\stackrel{\circ}{\Delta} f+\|\stackrel{\circ}{B}\|^{2} f\right]_{\alpha} \\
& \leq C\left(|f|_{C_{v}^{2, \alpha}}+\zeta_{r}\|\stackrel{\circ}{B}\| \cdot \zeta_{r}^{1+\alpha}[\stackrel{\circ}{B}]_{\alpha} \cdot \zeta_{r}^{-v}|f|+\zeta_{r}^{2}\|\stackrel{\circ}{B}\|^{2} \cdot \zeta_{r}^{\alpha-v}[f]_{\alpha}\right) \\
& \leq C|f|_{C_{v}^{2, \alpha}}
\end{aligned}
$$

Lemma 2.7 now shows that in any normal coordinate chart

$$
\begin{aligned}
\sum_{k=0}^{3} \zeta_{r}^{k-v}\left\|\nabla^{k}(\Theta(f)-f)\right\| & \leq C \sum_{k=0}^{3} \zeta_{r}^{-v}\|Y\|^{k}\left\|\nabla^{k}(\Theta(f)-f)\right\| \\
& \leq C r^{2} \sum_{k=0}^{3} \zeta_{r}^{-v}\|Y\|^{k}\left\|\nabla^{k} f\right\| \\
& \leq C r^{2}|f|_{C_{v}^{3}\left(\tilde{\Sigma}_{r}(\sigma, \delta)\right)}
\end{aligned}
$$

since $\zeta_{r}(x)^{-1}\|Y(x)\|$ is bounded above and below away from zero by a constant $C$ independent of $r, \varepsilon$ and $\delta$ for all $x \in \tilde{\Sigma}_{r}(\sigma, \delta)$. We deduce $|\Theta(f)-f|_{C_{v}^{2, \alpha}} \leq$ $C r^{2}|f|_{C_{v}^{2, \alpha}}$ from this. Finally, Lemma 2.2 shows that in any normal coordinate chart

$$
\begin{aligned}
\zeta_{r}^{2-v} \mid & \stackrel{\mathcal{L}}{\mathcal{L}}(f)-\mathcal{L}(f) \mid+\zeta_{r}^{2+\alpha-v}[\stackrel{\circ}{\mathcal{L}}(f)-\mathcal{L}(f)]_{\alpha} \\
& \leq C \zeta_{r}^{2}\left(\sum_{k=0}^{2} \zeta_{r}^{-v}\|Y\|^{k}\|\stackrel{\circ}{\nabla} f\|+\|Y\|^{2+\alpha} \zeta_{r}^{-v}\left[\stackrel{\circ}{\nabla}^{2} f\right]_{\alpha}\right) \\
& \leq C r^{2}|f|_{C_{v}^{2, \alpha}} .
\end{aligned}
$$

Putting together the estimates we've found leads to the desired result.

\subsubsection{The fixed-point argument}

We are now in a position to solve the $\mathrm{CMC}$ equation up to a finite-dimensional error by showing that the mapping $\mathcal{N}_{r}$ defined by equation (5.2) is a contraction mapping. We do so by establishing both estimates (5.3). Now, by Proposition 5.3 we know that $\left|H\left[\tilde{\Sigma}_{r}^{F}(\sigma, \delta)\right]-\frac{2}{r}\right|_{C_{v-2}^{0, \alpha}} \leq C \max \left\{r^{3-v}, r^{1-v} \varepsilon^{3 / 2-3 v / 4}, \delta r^{1-v} \varepsilon^{1-3 v / 4}\right\}:=$ $R(r, \varepsilon, \delta)$. To simplify the proof below, we will now assume that $\varepsilon \leq C r^{2}$ and $\delta \leq C r$. This will be justified in Section 6.3 when the parameters $\varepsilon_{k}$ and $\delta_{k}$ are determined. If we make this simplification, then $R(r, \varepsilon, \delta) \leq C r^{4-5 v / 2}$. When $v \in(1,6 / 5)$ then $R(r, \varepsilon, \delta)=o(r)$ which is needed so that $|f|_{C_{v}^{2, \alpha}}$ is small enough to invoke the various operator estimates used below. 
Proposition 5.9. There exists $w:=w_{r}(\sigma, \delta) \in C_{v-2}^{0, \alpha}\left(\tilde{\Sigma}_{r}^{F}(\sigma, \delta)\right)$ with $v \in(1,6 / 5)$ and $f:=f_{r}(\sigma, \delta) \in C_{v}^{2, \alpha}\left(\tilde{\Sigma}_{r}^{F}(\sigma, \delta)\right)$ defined by $f:=\frac{1}{r} \mathcal{R}\left(w-H\left[\tilde{\Sigma}_{r}^{F}(\sigma, \delta)\right]+\frac{2}{r}\right)$ so that

$$
H\left[\mu_{r f}\left(\tilde{\Sigma}_{r}^{F}(\sigma, \delta)\right)\right]-\frac{2}{r}=\mathcal{E}
$$

where $\mathcal{E} \in \tilde{\mathcal{W}}^{F}$. Moreover, the estimate

$$
|f|_{C_{v}^{2, \alpha}} \leq C r^{3-5 v / 2}
$$

holds for the function $f$, where the constant $C$ is independent of $r, \varepsilon$ and $\delta$. Finally, the mapping $(\sigma, \delta) \mapsto f(\sigma, \delta)$ is smooth in the sense of Banach spaces.

Proof. Let $w_{0}:=H\left[\tilde{\Sigma}_{r}(\sigma, \delta)\right]-\frac{2}{r}$. From Proposition 5.3 we know that $\left|w_{0}\right|_{C_{v-2}^{0, \alpha}} \leq$ $R(r, \varepsilon, \delta)$ when $r$ is sufficiently small. We now establish the first estimate (5.3a) as laid out in Section 5.1.1 when $r$ is sufficiently small. To do so, we consider the operator $\mathcal{N}_{r}$ in any normal coordinate chart and choose $w \in C_{\nu-2}^{0, \alpha}\left(\tilde{\Sigma}_{r}(\sigma, \delta)\right)$ with $|w|_{C_{v-2}^{0, \alpha}} \leq 2\left|w_{0}\right|_{C_{v-2}^{0, \alpha}}$. Using equation (5.4) and the results of Proposition 5.6, Proposition 5.7 and Proposition 5.8, we deduce

$$
\begin{aligned}
& \left|\mathcal{N}_{r}(w)\right|_{C_{v-2}^{0, \alpha}} \leq\left|(\stackrel{\circ}{\mathcal{L}} \circ \Theta-\mathcal{L})\left(\mathcal{R}\left(w-w_{0}\right)\right)\right|_{C_{\nu-2}^{0, \alpha}}
\end{aligned}
$$

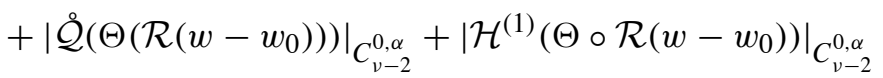

$$
\begin{aligned}
& \leq C r^{2}\left(\left|\mathcal{R}\left(w-w_{0}\right)\right|_{C_{v}^{2, \alpha}}+\left|\Theta\left(\mathcal{R}\left(w-w_{0}\right)\right)\right|_{C_{v}^{2, \alpha}}\right) \\
& +C r^{\nu-1}\left|\Theta\left(\mathcal{R}\left(w-w_{0}\right)\right)\right|_{C_{v}^{2, \alpha}}^{2} \\
& \leq C\left(r^{2}+r^{\nu-1} R(r, \varepsilon, \delta)\right)\left|w_{0}\right|_{C_{\nu-2}^{0, \alpha}} .
\end{aligned}
$$

We have also used the fact that $|\Theta(f)|_{C_{v}^{2, \alpha}} \leq C|f|_{C_{v}^{2, \alpha}}$ according to Lemma 2.7 and the fact that $|\mathcal{R}(f)|_{C_{v}^{2, \alpha}} \leq C|f|_{C_{\nu-2}^{0, \alpha}}$ according to Proposition 5.1. It is now clear that if we choose $r$ sufficiently small, it is possible to have $C\left(r^{2}+r^{\nu-1} R(r, \varepsilon, \delta)\right) \leq$ 2 which establishes the first estimate (5.3a). By performing essentially the exact same calculations, we find that the second estimate (5.3b) holds with this same value of $r$. Consequently, $\mathcal{N}_{r}$ is a contraction mapping on the ball of radius $2\left|w_{0}\right|_{C_{\nu-2}^{0, \alpha}}$. The size of the solution $f:=f(\sigma, \delta)$ follows from our bounds on $\mathcal{R}$; while the smoothness of the mapping $(\sigma, \delta) \mapsto f(\sigma, \delta)$ is a standard consequence of the contraction mapping theorem. Therefore the proposition follows.

\subsection{The one-ended surface}

\subsubsection{Strategy}

The strategy for solving the CMC equation (5.1) in the case of the one-ended surface $\tilde{\Sigma}_{r}^{O E}(\sigma, \delta)$ must be modified in order to take the non-compactness of $\tilde{\Sigma}_{r}^{O E}(\sigma, \delta)$ into 
account. In fact, the modification required can be understood by considering the outcome of the linear analysis, specifically the nature of the parametrix for $\mathcal{L}$. In this case, the outcome of the construction of the parametrix, which will mimic Proposition 5.1 as closely as possible, will be an operator $\mathcal{R}: C_{\nu-2, \bar{v}}^{0, \alpha}\left(\tilde{\Sigma}_{r}^{O E}(\sigma, \delta)\right) \rightarrow$ $C_{\nu, \bar{v}}^{2, \alpha}\left(\tilde{\Sigma}_{r}^{O E}(\sigma, \delta)\right) \oplus \tilde{\mathcal{V}}$ satisfying $\mathcal{L} \circ \mathcal{R}=i d+\mathcal{E}$. The operator $\mathcal{E}$ again maps into a finite-dimensional subspace $\tilde{\mathcal{W}}^{O E}$. The subspace $\tilde{\mathcal{V}}$ is the new ingredient, and it can be explained as follows. First, let $J_{\text {Del }}^{s}$ for $s=0,1$ be the bounded and linearly growing Jacobi fields of the standard Delaunay surface and define the space

$$
\tilde{\mathcal{V}}:=\operatorname{span}\left\{\chi_{\text {Del }}^{\bar{\tau}} J_{\text {Del }}^{1}, \chi_{\text {Del }}^{\bar{\tau}} J_{\text {Del }}^{2}\right\}
$$

where $\chi_{\text {Del }}^{\bar{\tau}}$ is a smooth, monotone cut-off function that transitions from zero to one in the neck region where the Delaunay end of $\tilde{\Sigma}_{r}^{O E}(\sigma, \delta)$ is attached to the finite part of $\tilde{\Sigma}_{r}^{O E}(\sigma, \delta)$. The reason $\tilde{\mathcal{V}}$ is needed is simply because $\mathcal{L}: C_{\nu, \bar{v}}^{2, \alpha}\left(\tilde{\Sigma}_{r}^{O E}(\sigma, \delta)\right) \rightarrow$ $C_{\nu-2, \bar{v}}^{0, \alpha}\left(\tilde{\Sigma}_{r}^{O E}(\sigma, \delta)\right)$ is not surjective but becomes so when growth like the first nondecaying Jacobi fields of $\mathcal{L}$ is permitted. But now the fact that one component of the solution of the linearized problem does not decay forces the modified approach that will be outlined in the next two paragraphs, since the quadratic remainder of the mean curvature will not behave appropriately for this component. An approach similar to the one proposed below has been used in [17].

To compensate for the non-decaying component of the solution of the linearized equation, one proceeds as follows. Let $\mathcal{R}^{(1)}$ denote the component of $\mathcal{R}$ mapping into $C_{\nu, \bar{v}}^{2, \alpha}\left(\tilde{\Sigma}_{r}^{O E}(\sigma, \delta)\right)$ and let $\mathcal{R}^{(2)}$ be the component of $\mathcal{R}$ mapping into $\tilde{\mathcal{V}}$. Furthermore, if $\mathcal{R}^{(2)}(w)=a_{1} \chi_{\text {Del }}^{\bar{\tau}} J_{\text {Del }}^{1}+a_{2} \chi_{\text {Del }}^{\bar{\tau}} J_{\text {Del }}^{2}$, then let us write $\mathcal{R}^{(2)}(w):=\left(a_{1}(w), a_{2}(w)\right)$ despite the slight abuse of notation that this represents. Now the equation that needs to be solved is still

$$
H\left[\mu_{r f}\left(\tilde{\Sigma}_{r}^{O E}(\sigma, \delta)\right)\right]=\frac{2}{r}
$$

but for $f \in C_{\nu-2, \bar{v}}^{0, \alpha}\left(\tilde{\Sigma}_{r}^{O E}(\sigma, \delta)\right)$. Recall that the last two free parameters of $\tilde{\Sigma}_{r}^{O E}(\sigma, \delta)$, namely $\sigma_{K}$ and $\delta_{K}$, parametrize asymptotically non-trivial deformations of $\tilde{\Sigma}_{r}^{O E}(\sigma, \delta)$. That is, these cause the period and location of the entire Delaunay end to change. The idea for converting (5.7) into a fixed-point problem is to associate $\mathcal{R}^{(1)}$ with $f$ and $\mathcal{R}^{(2)}$ with the parameters $\left(\sigma_{K}, \delta_{K}\right)$.

This can be done as follows. Recall that there are specific values $\sigma_{K}=\stackrel{\circ}{\sigma}_{K}$ and $\delta_{K}=0$ which produce optimal matching in the assembly of $\tilde{\Sigma}_{r}^{O E}(\sigma, \delta)$. With slight abuse of notation, write $\tilde{\Sigma}_{r}^{O E}(a):=\tilde{\Sigma}_{r}^{O E}(\sigma, \delta)$ with $a:=\left(a_{1}, a_{2}\right)$ and $\left(\sigma_{K}, \delta_{K}\right)=$ $\left(\stackrel{\circ}{\sigma}_{K}+a_{1}, a_{2}\right)$. Given the flexibility one has in the choice of $\tilde{\mathcal{V}}$, one can arrange that

$$
\left.\frac{\partial}{\partial a_{i}} H\left[\tilde{\Sigma}_{r}^{O E}\left(a_{1}, a_{2}\right)\right]\right|_{a=0}=\mathcal{L}\left(\chi_{\text {Del }}^{\bar{\tau}} J_{\text {Del }}^{i}\right)
$$


for $i=1,2$. Consequently, the Ansätze

$$
w_{0}:=H\left[\tilde{\Sigma}_{r}^{O E}(\sigma, \delta)\right]-\frac{2}{r} \quad f:=\frac{1}{r} \mathcal{R}^{(1)}\left(w-w_{0}\right) \quad a:=\mathcal{R}^{(2)}\left(w-w_{0}\right)
$$

transform equation (5.7) into

$$
\begin{aligned}
w- & H\left[\mu_{\mathcal{R}^{(1)}\left(w-w_{0}\right)}\left(\tilde{\Sigma}_{r}^{O E}\left(\mathcal{R}^{(2)}\left(w-w_{0}\right)\right)\right)\right]+\frac{2}{r}+\mathcal{E}\left(w-w_{0}\right) \\
= & w+\mathcal{E}\left(w-w_{0}\right) .
\end{aligned}
$$

To solve this problem (up to an error term) we show that the mapping $\mathcal{N}_{r}$ : $C_{\nu-2, \bar{\nu}}^{0, \alpha}\left(\tilde{\Sigma}_{r}^{O E}(\sigma, \delta)\right) \rightarrow \tilde{\Sigma}_{r}^{O E}(\sigma, \delta)$ given by the left hand side of (5.8) is a contraction mapping onto a neighbourhood of zero containing $H\left[\tilde{\Sigma}_{r}^{O E}(\sigma, \delta)\right]-\frac{2}{r}$. Doing so requires establishing the same kinds of estimates as (5.3) via the expansion (5.4) that is still valid here. Therefore the next four section mimic Sections 5.1.2-5.1.5. Once we have the necessary estimates, then we obtain a solution of the equation (5.7) up to a term in $\tilde{\mathcal{W}}^{O E}$. This finite-dimensional error term must of course still be dealt with in order to find a true CMC surface near to $\tilde{\Sigma}_{r}^{O E}(\sigma, \delta)$, and this will be carried out in Section 6 .

\subsubsection{The linear analysis}

The construction of the parametrix in the case of the one-ended surface begins with the definition of an additional set of partitions of unity for $\tilde{\Sigma}_{r}^{O E}(\sigma, \delta)$ as follows. For any $\bar{\tau} \in \mathbb{R}$ let $C y l_{\bar{\tau}}$ be the set $\{(x, t) \in M:\|x\| \leq r$ and $t \geq \bar{\tau}\}$, and let $\mathcal{D}^{\bar{\tau}}:=\tilde{\Sigma}_{r}^{O E}(\sigma, \delta) \cap C y l_{\bar{\tau}}$. Now define the smooth, monotone cut-off function $\chi_{\text {Del }}^{\bar{\tau}}$ that equals one in $\mathcal{D}^{\bar{\tau}}$ and vanishes in $\tilde{\Sigma}_{r}^{O E}(\sigma, \delta) \backslash \mathcal{D}^{\bar{\tau}-r}$.

A second important ingredient that will be used in the construction of the parametrix is a careful analysis of the properties of the linearized mean curvature operator of a near-degenerate Delaunay surface with respect to the $C_{v, \bar{v}}^{k, \alpha}$ norm. This was carried out in [10] and the relevant results from [10, Section 4] can be adapted to the needs of this paper and will be quoted whenever they are used in the proof given below.

Proposition 5.10. Let $(v, \bar{v}) \in(1,2) \times(-1,0)$.

There is an operator $\mathcal{R}: C_{\nu-2, \bar{v}}^{0, \alpha}\left(\tilde{\Sigma}_{r}^{O E}(\sigma, \delta)\right) \rightarrow C_{v, \bar{v}}^{2, \alpha}\left(\tilde{\Sigma}_{r}^{O E}(\sigma, \delta)\right) \oplus \tilde{\mathcal{V}}$ that satisfies $\mathcal{L} \circ \mathcal{R}=i d-\mathcal{E}$ where $\mathcal{E}: C_{v-2, \bar{v}}^{0, \alpha}\left(\tilde{\Sigma}_{r}^{O E}(\sigma, \delta)\right) \rightarrow \tilde{\mathcal{W}}^{O E}$. Here $\tilde{\mathcal{W}}^{O E}$ is a finitedimensional space that will be defined below. The estimates satisfied by $\mathcal{R}$ and $\mathcal{E}$ are

$$
|\mathcal{R}(w)|_{C_{v, \bar{v}}^{2, \alpha} \oplus \tilde{\mathcal{V}}}+|\mathcal{E}(w)|_{C_{0, \bar{\nu}}^{2, \alpha}} \leq C|w|_{C_{v-2, \bar{\nu}}^{0, \alpha}}
$$

for all $w \in C_{\nu-2}^{0, \alpha}\left(\tilde{\Sigma}_{r}^{O E}(\sigma, \delta)\right)$, where $C$ is a constant independent of $r, \varepsilon, \delta$ and $K$. 
Proof. Let $w \in C_{v-2, \bar{v}}^{0, \alpha}\left(\tilde{\Sigma}_{r}^{O E}(\sigma, \delta)\right)$ be given. The solution of the equation $\mathcal{L}(u)=$ $w+\mathcal{E}(w)$ will be constructed broadly along the same lines as in Proposition 5.1 in that local solutions on the spherical constituents, the necks and the Delaunay end of $\tilde{\Sigma}_{r}^{O E}(\sigma, \delta)$ will be patched together. In this case, however, a preliminary step is needed to reduce the interaction between the Delaunay end and the finite part of $\tilde{\Sigma}_{r}^{O E}(\sigma, \delta)$. This amounts to showing that one can assume that $w \equiv 0$ in a large part of $\tilde{\Sigma}_{r}^{O E}(\sigma, \delta)$.

Step 0. Define $w_{\text {mid }}:=\chi_{\text {Del }}^{t_{K}}\left(1-\chi_{\text {Del }}^{t_{2 K}}\right) w$. Using the methods of Proposition 5.1 , one can solve the Dirichlet problem $\mathcal{L}\left(u_{\text {mid }}\right)=w_{\text {mid }}+\mathcal{E}\left(w_{\text {mid }}\right)$ and $u_{\text {mid }}=$ 0 on $\partial\left[\mathcal{D}^{t_{K}} \backslash \mathcal{D}^{t_{2 K}}\right]$. The estimate one obtains is $\left|u_{\text {mid }}\right|_{C_{v}^{2, \alpha}}+\left|\mathcal{E}\left(w_{\text {mid }}\right)\right|_{C_{0}^{2, \alpha}} \leq$ $C\left|w_{\text {mid }}\right|_{C_{\nu-2}^{0, \alpha}} \leq C|w|_{C_{\nu-2, \bar{v}}^{0, \alpha}}$ where $C$ is independent of $r, \varepsilon$ and $K$. Then $u_{\text {mid }}$ can be extended to all of $\tilde{\Sigma}_{r}^{O E}(\sigma, \delta)$ by defining $\bar{u}_{\text {mid }}:=\chi_{\text {Del }}^{t_{K}-r}\left(1-\chi_{\text {Del }}^{t_{2 K}+r}\right) u_{\text {mid }}$. If one now solves $\mathcal{L}(u)=w-\mathcal{L}\left(\bar{u}_{\text {mid }}\right)$, then the function $u+u_{\text {mid }}$ solves $\mathcal{L}\left(u+u_{\text {mid }}\right)=w$. The advantage will be that the function $w-\mathcal{L}\left(\bar{u}_{\text {mid }}\right)$ vanishes in $\mathcal{D}^{t_{K}} \backslash \mathcal{D}^{t_{2 K}}$ but still satisfies the same estimates as did $w$. One can also assume that $K$ can be as large as desired.

Step 1. Let $w \in C_{\nu-2, \bar{v}}^{0, \alpha}\left(\tilde{\Sigma}_{r}^{O E}(\sigma, \delta)\right)$ be given and assume that $w \equiv 0$ in $\mathcal{D}^{t_{K}} \backslash$ $\mathcal{D}^{t_{2 K}}$. Consider the equation $\mathcal{L}\left(u_{\text {fin }}\right)=w_{\text {fin }}+\mathcal{E}\left(w_{\text {fin }}\right)$ where $w_{\text {fin }}:=\left(1-\chi_{\text {Del }}^{t_{2 K}}\right) w$ and view the function on the right hand side as being defined on the finite-length surface. Using the methods of Proposition 5.1, one can find a solution $u_{\text {fin }} \in$ $C_{v}^{2, \alpha}\left(\tilde{\Sigma}_{r}^{F}(\sigma, \delta)\right)$ satisfying $\left|u_{\mathrm{fin}}\right|_{C_{\nu}^{2, \alpha}}+\left.\mathcal{E}\left(w_{\mathrm{fin}}\right)\right|_{C_{0}^{0, \alpha}} \leq C|w|_{C_{\nu-2, \bar{\nu}}^{0, \alpha}}$ for some constant $C$ independent of $r, \varepsilon$ and $K$. Note that one must view $\mathcal{D}^{t_{K}} \backslash \mathcal{D}^{t_{2} K}$ as being very close to a union of spherical and neck regions in order to do so. Furthermore, by carefully analyzing the iteration process that leads to the solution, the fact that $\operatorname{supp}\left(w_{\text {fin }}\right) \subseteq \tilde{\Sigma}_{r}^{O E}(\sigma, \delta) \backslash \mathcal{D}^{t_{K}}$ implies that for $x \in \tilde{\Sigma}_{r}^{O E}(\sigma, \delta)$ near $\partial \mathcal{D}^{t_{2 K}}$ one has $\zeta_{r}^{-v}(x)\left|u_{\mathrm{fin}}(x)\right| \leq C \mathrm{e}^{-K}|w|_{C_{v-2, \bar{v}}^{0, \alpha}}$ for some constant $C$ independent of $r, \varepsilon$ and $K$. Extend $u_{\text {fin }}$ to all of $\tilde{\Sigma}_{r}^{O E}(\sigma, \delta)$ by defining $\bar{u}_{\text {fin }}:=\left(1-\chi_{\text {Del }}^{t_{2 K}+r}\right) u_{\text {fin }}$.

Step 2. For $w \in C_{\nu-2, \bar{v}}^{0, \alpha}\left(\tilde{\Sigma}_{r}^{O E}(\sigma, \delta)\right)$ from Step 1 , set $w_{\text {Del }}:=\chi_{\text {Del }}^{t_{2 K}} w$ and consider now the equation $\mathcal{L}_{D}\left(u_{\text {Del }}\right)=w_{\text {Del }}$ but viewed as an equation on the complete Delaunay surface $D_{r}$. Then using the methods of [10, Section 4] and [15] along with the condition $\bar{v} \in(-1,0)$, there is a solution of this equation which can be decomposed as $u_{\text {Del }}=v_{\text {Del }}+a_{\text {Del }}^{1} \chi_{\text {Del }}^{\bar{\tau}} J_{\text {Del }}^{1}+a_{\text {Del }}^{2} \chi_{\text {Del }}^{\bar{\tau}} J_{\text {Del }}^{2}$. Here $v_{\text {Del }} \in C_{v, \bar{v}}^{2, \alpha}\left(D_{r}\right)$ and one has the estimate $\left|v_{\text {Del }}\right|_{C_{\nu}^{2, \alpha}}+\left|a_{\text {Del }}^{1}\right|+\left|a_{\text {Del }}^{2}\right| \leq C\left|w_{\text {Del }}\right|_{C_{\nu-2, \bar{v}}^{0, \alpha}}$ for some constant independent of $r$ and $\varepsilon$. Furthermore, because $\operatorname{supp}\left(w_{\text {Del }}\right) \subseteq \tilde{\Sigma}_{r}^{O E}(\sigma, \delta) \cap$ $\mathcal{D}^{t_{K}}$ one can arrange to have $\zeta_{r}^{-v}(x)\left|u_{\mathrm{fin}}(x)\right| \leq C \mathrm{e}^{-K}|w|_{C_{\nu-2, \bar{v}}^{0, \alpha}}$ for $\bar{x} \in \tilde{\Sigma}_{r}^{O E}(\sigma, \delta)$ near $\partial \mathcal{D}^{t_{2 K}}$ for some constant $C$ independent of $r, \varepsilon$ and $K$. Now it is possible to view $\tilde{\Sigma}_{r}^{O E}(\sigma, \delta) \cap \mathcal{D}^{t_{K}}$ as a graph over $D_{r} \cap \mathcal{D}^{t_{K}}$ and hence $u_{\text {Del }}$ can be viewed as a function defined on $\tilde{\Sigma}_{r}^{O E}(\sigma, \delta) \cap \mathcal{D}^{t_{K}}$. Extend this function to all of $\tilde{\Sigma}_{r}^{O E}(\sigma, \delta)$ by defining $\bar{u}_{\text {Del }}:=\chi_{\text {Del }}^{t_{K}-r} u_{\text {Del }}$. 
Step 3. The estimate of $\mathcal{L}(u)-w$ up to a finite-dimensional error term proceeds in the same way as in Step 3 of Proposition 5.1. The extra exponential decay of $\bar{u}_{\text {fin }}$ and $\bar{u}_{\text {Del }}$ near $\partial\left[\mathcal{D}^{t_{K}} \backslash \mathcal{D}^{t_{2 K}}\right]$ ensures that the cut-off errors that arise there are small. Consequently one can iterate the steps above and find the desired solution $\mathcal{R}(w) \in C_{\nu, \bar{v}}^{2, \alpha}\left(\tilde{\Sigma}_{r}^{O E}(\sigma, \delta)\right) \oplus \tilde{\mathcal{V}}$ and satisfying the desired estimate.

The definition of the finite-dimensional image of the map $\mathcal{E}: C_{\nu-2}^{0, \alpha}\left(\tilde{\Sigma}_{r}^{O E}(\sigma, \delta)\right) \rightarrow$ $\tilde{\mathcal{W}}^{O E}$ is once again a by-product of the previous proof.

Definition 5.11. Define

$$
\tilde{\mathcal{W}}^{O E}:=\operatorname{span}\left\{\chi_{\mathrm{ext}, k}^{\tau_{1}} J_{k}, \chi_{\mathrm{ext}, k}^{\tau_{1}} \mathcal{L}_{S}\left(\eta_{k}^{+} \chi_{\text {neck }, k}^{\tau_{1}}\right): k=0, \ldots, K-1\right\} \cup\left\{\chi_{\mathrm{ext}, K}^{\tau_{1}} J_{K}\right\}
$$

\subsubsection{The mean curvature estimate}

The estimate of the mean curvature of the one-ended surface $\tilde{\Sigma}_{r}^{O E}(\sigma, \delta)$ is a very straightforward extension of the results obtained in Section 5.1.3. In fact, the result is the same.

Proposition 5.12. Suppose $v \in(1,2)$ and $\bar{v} \in(-1,0)$ is sufficiently close to zero. The mean curvature of $\tilde{\Sigma}_{r}^{O E}(\sigma, \delta)$ satisfies the estimate

$$
\left|H-\frac{2}{r}\right|_{C_{v-2, \bar{v}}^{0, \alpha}} \leq C \max \left\{r^{3-v}, r^{1-v} \varepsilon^{3 / 2-3 v / 4}, \delta r^{1-v} \varepsilon^{1-3 v / 4}\right\}
$$

for some constant $C$ independent of $r, \varepsilon, \delta$ and $K$.

Proof. The computations of Proposition 5.3 give the estimate of the mean curvature of the part $\tilde{\Sigma}_{r}^{O E}(\sigma, \delta)$ constructed from spheres and catenoids. It thus remains only to compute the estimate of the mean curvature of $\tilde{D}_{r}^{+}\left(\sigma_{K}, \delta_{K}\right)$ (actually, the part of $\tilde{D}_{r}^{+}\left(\sigma_{K}, \delta_{K}\right)$ that is an un-perturbed Delaunay surface). Once again, the key is to use Lemma 2.3 , but this time realizing that the mean curvature of $\tilde{D}_{r}^{+}\left(\sigma_{K}, \delta_{K}\right)$ with respect to the Euclidean background metric in a tubular neighbourhood of $\gamma$ is exactly equal to $\frac{2}{r}$. Let $p$ be any point on $\gamma$ and let $p^{\prime}$ be any point on $\tilde{D}_{r}^{+}\left(\sigma_{K}, \delta_{K}\right) \cap$ $B_{r}(p)$. Then by the lemma,

$$
\left|H\left(p^{\prime}\right)-\frac{2}{r}\right| \leq C|R(p)|\left(\left\|Y\left(p, p^{\prime}\right)\right\|^{2}\left\|\stackrel{\circ}{B}\left(p^{\prime}\right)\right\|+\left\|Y\left(p, p^{\prime}\right)\right\|\right)
$$

where $R$ is an expression that is linear in the components of the ambient Riemannian curvature at $p$ and $Y\left(p, p^{\prime}\right)$ is the position vector field of $p^{\prime}$ with respect to $p$. The constant $C$ is independent of $r$ and $\varepsilon$. Since the curvature is exponentially decaying along $\gamma$, the same estimates from Proposition 5.3 continue to hold and yield the estimate needed here. 


\subsubsection{The operator estimates}

The estimates for the quantities $\left|\mathcal{Q}\left(f_{1}\right)-\stackrel{\mathcal{Q}}{(}\left(f_{2}\right)\right|$ and $\left|\mathcal{H}^{(1)}\left(f_{1}\right)-\mathcal{H}^{(1)}\left(f_{2}\right)\right|$ and $\mid \mathcal{L}_{\circ}$ $\Theta(f)-\mathcal{L}(f) \mid$ in the $C_{v-2, \bar{v}}^{0, \alpha}$ norm needed to show that the map $\mathcal{N}_{r}$ is a contraction mapping in the case of the one-ended surface are very similar to the analogous estimates for the finite-length surface. First, the result of Lemma 5.5 continues to hold because the calculations are essentially identical, the only difference being the need to multiply by factors of $\mathrm{e}^{-\bar{v} T}$ along the end of $\tilde{\Sigma}_{r}^{O E}(\sigma, \delta)$. These growing factors are compensated for by the exponential decay assumed for $f$. Consequently it is possible to make $\|B\||f|+\|\nabla f\|$ pointwise small everywhere by choosing $|f|_{C_{\nu, \bar{v}}^{2, \alpha}}$ sufficiently small.

Next, the non-linear estimate analogous to Propositions 5.6-5.8 follow similarly because all terms coming from the background metric decay exponentially. One has the following results.

Proposition 5.13. There exists $M>0$ so that if $f_{1}, f_{2} \in C_{\nu, \bar{v}}^{2, \alpha}\left(\tilde{\Sigma}_{r}^{O E}(\sigma, \delta)\right)$ for $v \in$ $(1,2)$ and $\bar{v} \in(-1,0)$ sufficiently close to zero and satisfying $\left|f_{1}\right|_{C_{\nu, \bar{v}}^{2, \alpha}}+\left|f_{2}\right|_{C_{\nu, \bar{v}}^{2, \alpha}} \leq$ $M$, then the quadratic remainder term $\mathcal{Q}$ satisfies the estimate

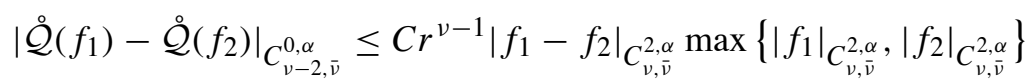

where $C$ is a constant independent of $r, \varepsilon$ and $\delta$.

Proposition 5.14. There exists $M>0$ so that if $f_{1}, f_{2} \in C_{\nu, \bar{v}}^{2, \alpha}\left(\tilde{\Sigma}_{r}^{O E}(\sigma, \delta)\right)$ for $v \in$ $(1,2)$ and $\bar{v} \in(-1,0)$ sufficiently close to zero and satisfying $\left|f_{1}\right|_{C_{v}^{2, \alpha}}+\left|f_{2}\right|_{C_{v}^{2, \alpha}} \leq$ $M$, then

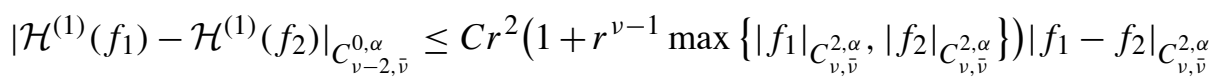

where $C$ is a constant independent of $r, \varepsilon$ and $\delta$.

Proposition 5.15. There exists $M>0$ so that if $f \in C_{v, \bar{v}}^{2, \alpha}\left(\tilde{\Sigma}_{r}^{O E}(\sigma, \delta)\right)$ for $v \in$ $(1,2)$ and $\bar{v} \in(-1,0)$ sufficiently close to zero and satisfying $|f|_{C_{v}^{2, \alpha}} \leq M$, then

$$
|\mathcal{L} \circ \Theta(f)-\mathcal{L}(f)|_{C_{\nu-2, \bar{\nu}}^{0, \alpha}} \leq C r^{2}|f|_{C_{\nu, \bar{v}}^{2, \alpha}}
$$

where $C$ is a constant independent of $r, \varepsilon$ and $\delta$.

\subsubsection{The fixed-point argument}

The fixed-point argument in the case of the one-ended surface is again essentially identical to the argument in the case of the finite-length surface with the exception that dealing with the non-decaying component of the parametrix requires some additional care. As before, let $w_{0}:=H\left[\tilde{\Sigma}_{r}^{O E}(\sigma, \delta)\right]-\frac{2}{r}$ and $R(r, \varepsilon, \delta):=$ $\max \left\{r^{3-v}, r^{1-v} \varepsilon^{3 / 2-3 v / 4}, \delta r^{1-v} \varepsilon^{1-3 v / 4}\right\}$. We will once again assume that $\varepsilon \leq$ $C r^{2}$ and $\delta \leq C r$ so that when $v \in(1,6 / 5)$ then $R(r, \varepsilon, \delta) \leq C r^{4-5 v / 2}$. 
Proposition 5.16. There exists $w:=w_{r}(\sigma, \delta) \in C_{\nu-2, \bar{v}}^{0, \alpha}\left(\tilde{\Sigma}_{r}^{O E}(\sigma, \delta)\right)$ with $v \in$ $(1,6 / 5)$ and $\bar{v} \in(-1,0)$ sufficiently close to zero, along with $f:=f_{r}(\sigma, \delta) \in$ $C_{v, \bar{v}}^{2, \alpha}\left(\tilde{\Sigma}_{r}^{O E}(\sigma, \delta)\right)$ and $\left(a_{1}, a_{2}\right) \in \tilde{\mathcal{V}}$ defined by

$$
f:=\frac{1}{r} \mathcal{R}^{(1)}\left(w-H\left[\tilde{\Sigma}_{r}^{O E}(\sigma, \delta)\right]+\frac{2}{r}\right)
$$

and

$$
\left(a_{1}, a_{2}\right):=\mathcal{R}^{(2)}\left(w-H\left[\tilde{\Sigma}_{r}^{O E}(\sigma, \delta)\right]+\frac{2}{r}\right)
$$

so that

$$
H\left[\mu_{r f}\left(\tilde{\Sigma}_{r}^{O E}\left(a_{1}, a_{2}\right)\right)\right]-\frac{2}{r}=\mathcal{E}
$$

where $\mathcal{E} \in \tilde{\mathcal{W}}^{O E}$. Moreover, the estimate

$$
|f|_{C_{\nu, \bar{v}}^{2, \alpha}} \leq C r^{3-5 v / 2}
$$

holds for the function $f$, where the constant $C$ is independent of $r, \varepsilon$ and $\delta$. Finally, the mapping $(\sigma, \delta) \mapsto f(\sigma, \delta)$ is smooth in the sense of Banach spaces.

\section{Solving the finite-dimensional problem}

\subsection{Strategy}

It has now been established for both the finite-length and one-ended families of surfaces that if $r, \sigma$ and $\delta$ are sufficiently small, one can find $w_{r}(\sigma, \delta) \in C_{*}^{0, \alpha}\left(\tilde{\Sigma}_{r}(\sigma, \delta)\right)$ and corresponding $f_{r}(\sigma, \delta) \in C_{*}^{2, \alpha}\left(\tilde{\Sigma}_{r}(\sigma, \delta)\right)$ so that

$$
H\left[\mu_{f_{r}(\sigma, \delta)}\left(\tilde{\Sigma}_{r}(\sigma, \delta)\right)\right]-\frac{2}{r}=\mathcal{E}_{r}(\sigma, \delta)
$$

where $\mathcal{E}_{r}(\sigma, \delta)$ is an error term belonging to the finite-dimensional space $\tilde{\mathcal{W}}^{*}$ and depending on the free parameters in $\tilde{\Sigma}_{r}(\sigma, \delta)$. To complete the proof of the main theorem, we must show that it is possible to find a solution where these error terms vanish identically.

To proceed, let us consider the balancing map defined by

$$
\mathcal{B}_{r}(\sigma, \delta):=\pi\left(\mathcal{E}_{r}(\sigma, \delta)\right)
$$

where $\pi: \tilde{\mathcal{W}}^{*} \rightarrow \mathbb{R}^{d}$ is a suitable bounded projection operator and $d$ is the dimension of $\tilde{\mathcal{W}}^{*}$. (The operator $\pi$ will be a certain bijective $L^{2}$-orthogonal projection 
onto a finite-dimensional vector space.) Note that $\mathcal{B}_{r}$ is a smooth map between finite-dimensional vector spaces by virtue of the fact that the dependence of the solution $f_{r}(\sigma, \delta)$ on $(\sigma, \delta)$ is smooth and the mean curvature operator is a smooth map of the Banach spaces upon which it is defined. It will be shown using the implicit function theorem for finite-dimensional maps that for every sufficiently small $r>0$, there exists $(\sigma, \delta):=(\sigma(r), \delta(r))$ for which $\mathcal{B}_{r}(\sigma, \delta) \equiv 0$ identically. The precise nature of the scalar curvature of the background manifold $M$ enters the picture exactly here, since it will guide the selection of the parameters $\sigma$ and $\delta$ to first approximation.

\subsection{The balancing formula}

The projection operators that will be used to study the finite-dimensional error $\mathcal{E}_{r}(\sigma, \delta)$ can be defined as follows. For $k=0, \ldots, K$ let $J_{k}: S_{k} \rightarrow \mathbb{R}$ have its usual meaning; and let $I_{k}: r \varepsilon_{k} N_{k} \rightarrow \mathbb{R}$ be the function defined by $I_{k}(x):=$ $\|x\|\left(\|x\|^{2}-\varepsilon_{k}^{2}\right)^{-1 / 2}$ using the coordinates of the neck introduced in Section 3. This latter function is in the kernel of the linearized mean curvature operator of the catenoid with respect to the Euclidean background metric; it is an odd function with respect to the center of the catenoid and is asymptotic to \pm 1 . Now for convenience let $f:=f_{r}(\sigma, \delta)$ and $\Sigma_{f}:=\mu_{r f}\left(\tilde{\Sigma}_{r}(\sigma, \delta)\right)$ denote the solution found in the previous section and define $\pi_{k}: \tilde{\mathcal{W}}^{*} \rightarrow \mathbb{R}^{2}$ by

$$
\pi_{k}(e):=\left(\int_{\Sigma_{f}} e \cdot \chi_{\text {neck }, k}^{\tau_{1}} I_{k} \mathrm{dVol}_{g}, \int_{\Sigma_{f}} e \cdot \chi_{\mathrm{ext}, k}^{\tau_{4}} J_{k} \mathrm{dVol} g\right)
$$

The notation for the cut-off functions from Section 5.1.2 has been used here. A consequence of the following lemma is that if $\pi(e)=0$ then $e=0$. The proof is a straightforward computation.

Lemma 6.1. Choose $e \in \tilde{\mathcal{W}}$ and write $e=\sum_{k=1}^{K}\left(a_{k} \chi_{\text {ext }, k}^{\tau_{1}} J_{k}+b_{k} \chi_{\text {ext, }, k}^{\tau_{1}} \mathcal{L}_{S}\left(\eta_{k}^{+} \chi_{\text {neck, } k}^{\tau_{1}}\right)\right)$ for some $a_{k}, b_{k} \in \mathbb{R}$. Then

$$
\pi_{k}(e)=\left(C_{1} b_{k}+C_{1}^{\prime} r^{2}\left(\varepsilon_{k}^{3 / 2} a_{k}-\varepsilon_{k+1}^{3 / 2} a_{k+1}\right), C_{2} r^{2} a_{k}\right)
$$

where $C_{1}, C_{1}^{\prime}, C_{2}$ are constants independent of $r, \varepsilon$ and $\delta$.

A fundamental application of the expansions of the mean curvature found in Lemma 2.3 and equation (2.4) from Section 2.1 is to derive a formula relating $\pi\left(\mathcal{E}_{r}(\sigma, \delta)\right.$ to the geometry of $\tilde{\Sigma}_{r}(\sigma, \delta)$. This is in effect the balancing formula discovered by Korevaar, Kusner and Solomon [8]. It is via this formula that the location of the spheres and necks in $\tilde{\Sigma}_{r}(\sigma, \delta)$ and the background geometry of $M$ conspire to determine when a nearby CMC surface can be found. 
Proposition 6.2. Let $\Sigma_{f}:=\mu_{f}\left(\Sigma_{r}(\sigma, \delta)\right)$ and $f:=f_{r}(\sigma, \delta)$ for convenience. Then the mean curvature of $\Sigma_{f}$ satisfies the formula

$$
\begin{aligned}
& \int_{\Sigma_{f}}\left(H\left[\Sigma_{f}\right]-\frac{2}{r}\right) \chi_{\text {neck }, k}^{\tau_{1}} I_{k} \mathrm{dVol}_{g} \\
& =C_{0} \delta_{k} r \varepsilon^{3 / 2}+\mathcal{O}\left(\varepsilon^{11 / 4} r^{2}\right)+\mathcal{O}\left(r^{2} \varepsilon^{3 / 2}\right)+\mathcal{O}\left(r^{\nu} R(r, \varepsilon, \delta) \varepsilon^{2}\right) \\
& \int_{\Sigma_{f}}\left(H\left[\Sigma_{f}\right]-\frac{2}{r}\right) \chi_{\mathrm{ext}, k}^{\tau_{4}} J_{k} \mathrm{dVol}_{g} \\
& =r\left(C_{1} \varepsilon_{k+1}+C_{1}^{\prime} \varepsilon_{k+1}^{2}\right)-r\left(C_{1} \varepsilon_{k}+C_{1}^{\prime} \varepsilon_{k}^{2}\right)-C_{2} r^{4} \dot{S}\left(p_{k}\right) \\
& \quad+\mathcal{O}\left(\varepsilon r^{3}\right)+\mathcal{O}\left(r^{2} \varepsilon^{3 / 2}\right)+\mathcal{O}\left(r^{2} R(r, \varepsilon, \delta)\right)
\end{aligned}
$$

where $C_{1}, C_{1}^{\prime}, C_{2}$ are constants independent of $r, \varepsilon$ and $\delta$; and $\dot{S}\left(p_{k}\right):=$ $g\left(\nabla_{\dot{\gamma}\left(t_{k}\right)} S\left(p_{k}\right), \dot{\gamma}\left(t_{k}\right)\right)$ is the component of the gradient of the scalar curvature of the ambient metric along the geodesic $\gamma$ at $p_{k}$.

Proof. The formula (6.2b) will be derived first. Consider the subset $\mathcal{U}_{k}$ of $\Sigma_{0}$ consisting of the $k^{\text {th }}$ spherical region of $\Sigma:=\tilde{\Sigma}_{r}(\sigma, \delta)$ and its adjoining transition regions. Let $X=\frac{\partial}{\partial t}$ and $\tilde{X}=\chi_{\text {ext, } k}^{\tau_{4}} X$ using the normal coordinate system centered at $p_{k}$ used in the definition of $\Sigma \cap \mathcal{U}_{k}$. Furthermore, define the domain $\mathcal{W}_{k}$ in $M$ by requiring $\partial \mathcal{W}_{k}=\mathcal{U}_{k} \cup c_{1} \cup c_{2}$ where $c_{1}$ and $c_{2}$ are small embedded twodimensional disks with boundaries $\partial c_{1}$ and $\partial c_{2}$ contained in $t=$ constant planes

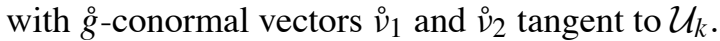

Now let $\Sigma^{\prime}$ be either $\Sigma_{f} \cap \mathcal{U}_{k}$ or $\Sigma \cap \mathcal{U}_{k}$; let $X^{\prime}$ be a vector field supported on this surface; and let $g^{\prime}$ be any choice of background metric. Define $I\left(\Sigma^{\prime}, X^{\prime}, g^{\prime}\right)$ to be the integral in (6.2b) except with $\Sigma_{f}$ replaced by $\Sigma^{\prime}$ and $J_{k}$ replaced by $g^{\prime}\left(X^{\prime}, N^{\prime}\right)$ where $N^{\prime}$ is the $g^{\prime}$-unit normal vector field of $\Sigma^{\prime}$ and the mean curvature and volume form calculated from $g^{\prime}$. It is now simple to phrase the means by which the formula (6.2b) will be found. First, $I\left(\Sigma_{f}, \tilde{X}, g\right)$ can be expressed as

$$
\begin{aligned}
I\left(\Sigma_{f}, \tilde{X}, g\right)= & I(\Sigma, X, \stackrel{\circ}{g})+(I(\Sigma, X, g)-I(\Sigma, X, \stackrel{\circ}{g})) \\
& +\left(I\left(\Sigma_{f}, \tilde{X}, g\right)-I(\Sigma, \tilde{X}, g)\right)+(I(\Sigma, \tilde{X}, g)-I(\Sigma, X, g)) .
\end{aligned}
$$

Then one can apply the first variation formula in Euclidean space to the first term, yielding a pair of boundary integrals; one can apply the expansions for the mean curvature with respect to the perturbed background metric from Lemma 2.3 to the second term, yielding a curvature quantity; and one can treat the third and fourth terms as small errors.

The details of the computation outlined above are as follows. For the first term, the classical first variation formula for a surface with boundary in Euclidean space 
gives

$$
\begin{aligned}
\int_{\Sigma \cap \mathcal{U}_{k}}\left(\stackrel{\circ}{H}[\Sigma]-\frac{2}{r}\right) J_{k} \mathrm{dVol}_{0} & \left.=\sum_{s=1}^{2}(-1)^{s}\left(\int_{\partial c_{s}} \stackrel{\circ}{g}\left(X, \stackrel{\circ}{\nu}_{s}\right) \mathrm{dL}_{0}+\frac{2}{r} \int_{c_{s}} g\left(X, \frac{\partial}{\partial t}\right) \mathrm{dVol}\right)_{0}\right) \\
& =\sum_{s=1}^{2}(-1)^{s} r\left(C_{1} \varepsilon_{s}+C_{1}^{\prime} \varepsilon_{s}^{2}\right)
\end{aligned}
$$

by direct computation, where $\varepsilon_{s}$ is the neck scale parameter associated to the neck adjoining the curve $\partial c_{s}$, while $C_{1}$ and $C_{1}^{\prime}$ are constants independent of $r, \varepsilon$ and $\delta$. For the second term, the expansion in equation 2.4 implies

$$
\begin{aligned}
& I(\Sigma, X, g)-I(\Sigma, X, \stackrel{\circ}{g}) \\
& =\int_{\Sigma \cap \mathcal{U}_{k}} \stackrel{\circ}{g}(X, \stackrel{\circ}{N})\left(\left(\frac{1}{6} \operatorname{Ric}(Y, Y)+\frac{1}{12} \bar{\nabla}_{Y} \operatorname{Ric}(Y, Y)\right)\left(\stackrel{\circ}{H}-\frac{2}{r}\right)\right. \\
& \quad-\left(\frac{1}{3} \operatorname{Rm}\left(E_{i}, Y, E_{j}, Y\right)+\frac{1}{6} \bar{\nabla}_{Y} \operatorname{Rm}\left(E_{i}, Y, E_{j}, Y\right)\right) \stackrel{\circ}{B}^{i j} \\
& \quad-\frac{2}{3} \operatorname{Ric}(\stackrel{\circ}{N}, Y)-\frac{1}{2} \bar{\nabla}_{Y} \operatorname{Ric}(\stackrel{\circ}{N}, Y) \\
& \left.\quad+\frac{1}{12} \bar{\nabla}_{N} \operatorname{Ric}(Y, Y)-\frac{1}{6} \bar{\nabla}_{N} \operatorname{Rm}(\stackrel{\circ}{N}, Y, \stackrel{\circ}{N}, Y)\right) \mathrm{dVol}_{0}+\mathcal{O}\left(r^{5}\right)
\end{aligned}
$$

where $Y$ is the position vector field of $\Sigma_{k}$, while the quantities $g, N, H, B$ and $\stackrel{\circ}{g}, \stackrel{\circ}{N}, \stackrel{\circ}{H}, \stackrel{\circ}{B}$ have their usual meanings. Since $\mathcal{U}_{k}$ is the normal graph of the function $r G$ over the sphere $S_{k}$ as in Section 3, one can replace the integral in (6.4) with an integral over $S_{k}$, at the expense of an error of size $\mathcal{O}\left(\varepsilon|\log (\varepsilon)| r^{3}\right)$. Hence by direct computation using $\stackrel{\circ}{H}=\frac{2}{r}$ and $\stackrel{\circ}{B}_{i j}=r \stackrel{\circ}{h}_{i j}$ one finds

$$
I(\Sigma, X, g)-I(\Sigma, X, \stackrel{\circ}{g})=-C_{2} r^{4} \dot{S}\left(p_{k}\right)+\mathcal{O}\left(\varepsilon|\log (\varepsilon)| r^{3}\right)
$$

where $C_{2}>0$ is a constant independent of $r, \varepsilon$ and $\delta$. An similar computation is performed in [20].

It remains to estimate the error terms appearing in (6.3). In the third term, the fact that $H\left[\Sigma_{f}\right]=H[\Sigma]+\dot{\mathcal{L}}(r f)+\stackrel{\mathcal{Q}}{(r f)}+\mathcal{H}(r f)$ must be used. Using the estimates for $H[\Sigma]$ and $\mathcal{Q}$ and $\mathcal{H}$ from Propositions 5.3, 5.7 and 5.8 along with the estimate of $f$ from Proposition 5.9, we find

$$
\left|I\left(\Sigma_{f}, \tilde{X}, g\right)-I(\Sigma, \tilde{X}, g)\right| \leq C r^{2}|f|_{C_{0}^{2, \alpha}\left(\Sigma \cap \mathcal{U}_{k}\right)} \leq C r^{2} R(r, \varepsilon, \delta) .
$$


Finally, for the fourth term, observe that $\tilde{X}-X$ is supported in a collar of width $\mathcal{O}\left(r \varepsilon^{3 / 4}\right)$ around the transition regions of $\Sigma \cap \mathcal{U}_{k}$. Hence

$$
|I(\Sigma, \tilde{X}, g)-I(\Sigma, X, g)| \leq C r^{2} \varepsilon^{3 / 2}
$$

using the estimate from Step 3 of Proposition 5.3 for the mean curvature in the transition region.

The derivation of the formula $(6.2 \mathrm{a})$ is similar to what has been done above. That is, writing $I\left(\Sigma^{\prime}, X^{\prime}, g^{\prime}\right)$ as before, but with $X^{\prime}$ equal to either $X:=\frac{\partial}{\partial t}$ or $\tilde{X}:=\chi_{\text {neck, } k}^{\tau_{1}} \frac{\partial}{\partial t}$, one finds the same decomposition as $(6.3)$ for $I\left(\Sigma_{f}, \tilde{X}, g\right)$. But now,

$$
\int_{\Sigma \cap \mathcal{N}_{k}^{\tau_{1}}}\left(\stackrel{\circ}{H}[\Sigma]-\frac{2}{r}\right) I_{k} \mathrm{dVol}_{0}=-\frac{2}{r} \int_{\Sigma \cap \mathcal{N}_{k}^{\tau_{1}}} I_{k} \mathrm{dVol}_{0}=C \delta_{k} r \varepsilon_{k}^{3 / 2}
$$

where $\delta_{k}$ is the displacement parameter of the neck $\mathcal{N}_{k}$ and $C$ is a constant independent of $r, \varepsilon$ and $\delta$. This is because $\int_{\Sigma \cap \mathcal{N}_{k}^{\tau_{1}}} \stackrel{\circ}{H}[\Sigma] I_{k} \mathrm{dVol}_{0}=0$ exactly (this is the first variation formula for the exactly minimal surface $\left.\Sigma \cap \operatorname{supp}\left(\chi_{\text {neck, },}^{\tau_{1}}\right)\right)$ and $I_{k}$ is an odd function with respect to the neck having $\delta_{k}=0$, whereas the integral is being taken over the neck with $\delta_{k} \neq 0$. The remaining terms in the expansion of $I\left(\Sigma_{f}, \tilde{X}, g\right)$ are small error terms whose estimates are sufficiently similar to the analogous ones above and will not be repeated for the sake of brevity.

\subsection{Proof of the main theorem}

The formulæ developed for the balancing map $\mathcal{B}_{r}: \mathbb{R}^{d} \rightarrow \mathbb{R}^{d}$ in the previous section make it possible to choose an exactly CMC surface from amongst the family of surfaces $\mu_{r f_{r}(\sigma, \delta)}\left(\tilde{\Sigma}_{r}(\sigma, \delta)\right)$. This will be done as follows. First, because of Lemma 6.1 , it is sufficient to find $(\sigma, \delta)$ so that the right hand sides of equations (6.2a) and (6.2b) vanish for every $k$. One should realize that $p_{k}$ in these equations can be expressed in terms of $\varepsilon_{1}, \varepsilon_{2}, \ldots$ via the formula $p_{k}:=\gamma\left(2 k r+\sum_{l=1}^{k} \varepsilon_{l}\right)$ and $\varepsilon_{k}$ can be expressed in terms of $\sigma_{k}$ via the formula $\sigma_{k}:=\Lambda_{k}\left(\varepsilon_{k}\right)$ as in Section 3. (In the case of the one-ended surface, let the relationship $\varepsilon_{K}=\rho_{T}(0)$ for $T=$ $2+\sigma_{K} / r$ satisfied by the Delaunay end of $\tilde{\Sigma}_{r}^{O E}(\sigma, \delta)$ be written $\varepsilon_{K}:=\Lambda_{K}\left(\sigma_{K}\right)$ for consistency.) Note that $\sigma_{k}$ and $p_{k}$ are smooth functions of $\varepsilon_{k}$. Finding the appropriate value $(\sigma, \delta)$ will amount to applying the implicit function theorem for smooth maps between finite dimensional spaces to this system of equations, and will lead to a unique solution $(\sigma, \delta):=(\sigma(r), \delta(r))$ for all sufficiently small $r>0$ and $\varepsilon, \delta$ satisfying $r^{4}<\varepsilon<r^{2}$ and $\delta<\varepsilon^{1 / 2}$.

The finite-length surface. The equations that must be solved to produce the finite-length CMC surface are as follows: if the various error quantities appearing in equations (6.2), divided by $r$, are denoted $E_{s, k}(r, \varepsilon)$ where $s=1$ refers to a 
neck and $s=2$ refers to a sphere, then

$$
\begin{aligned}
0 & =\delta_{1}+\varepsilon_{1}^{-3 / 2} E_{1,1}(r, \varepsilon, \delta) \\
& \vdots \\
0 & =\delta_{K-1}+\varepsilon_{K}^{-3 / 2} E_{1, K-1}(r, \varepsilon, \delta)
\end{aligned}
$$

as well as

$$
\begin{aligned}
0 & =-q_{1}\left(\varepsilon_{1}\right)+q_{2}\left(\varepsilon_{2}\right)-C_{2,1} r^{3} \dot{S}\left(p_{1}\right)+E_{2,1}(r, \varepsilon, \delta) \\
& \vdots \\
0 & =-q_{k}\left(\varepsilon_{k}\right)+q_{k+1}\left(\varepsilon_{k+1}\right)-C_{2, k} r^{3} \dot{S}\left(p_{k}\right)+E_{2, k}(r, \varepsilon, \delta) \\
& \vdots \\
0 & =-q_{K}\left(\varepsilon_{K}\right)-C_{2, K} r^{3} \dot{S}\left(p_{K}\right)+E_{2, K}(r, \varepsilon, \delta)
\end{aligned}
$$

where $q_{k}(\varepsilon):=C_{1, k} \varepsilon+C_{1, k}^{\prime} \varepsilon^{2}$ and $p_{k}:=\gamma\left(2 k r+\sum_{l=1}^{k} \varepsilon_{l}\right)$ while $C_{1, k}, C_{1, k}^{\prime}$ and $C_{2, k}$ are various constants independent of $r, \varepsilon$ and $\delta$. Note that the $E_{s, k}$ are smooth functions of $\varepsilon$. Also, because the $t \mapsto-t$ symmetry that has been imposed since the beginning, the scalar curvature must have a critical point at $p_{0}$.

One should now view the equations in (6.5) as a systems of equations for the $\varepsilon$ and $\delta$ variables depending on the parameter $r$ that is to be treated using the implicit function theorem. When $r=0$ there is an exact solution $\delta_{1}=\cdots=\delta_{K-1}=0$ and $\varepsilon_{1}=\cdots=\varepsilon_{K}=0$. Furthermore, it is easy to see that the derivative matrix of the function $\Phi(\varepsilon, \delta, r)$ defined by the right hand sides of (6.5a) and (6.5b) in the $\varepsilon$ and $\delta$ variables is invertible at $r=0$ with a lower bound of size $\mathcal{O}(1)$ on its determinant (the derivative matrix is upper-triangular with non-zero constants of size $\mathcal{O}(1)$ on the diagonal). Hence by the inverse function theorem there is a solution of (6.5) for all sufficiently small $r$, and the dependence of $\varepsilon$ and $\delta$ on $r$ is smooth. Note that the solution for small $r$ will have $\varepsilon_{k}=\mathcal{O}\left(r^{3} \sum_{k^{\prime}=0}^{k} S\left(p_{k^{\prime}}\right)\right)$ and hence $C_{1} r^{4} \leq \varepsilon_{k} \leq C_{2} r^{2}$ for numerical constants $C_{1}$ and $C_{2}$ if we assume that $\dot{S}(0)=0$. This is because the sum $\left.r \sum_{k^{\prime}=0}^{k} \dot{S}\left(p_{k^{\prime}}\right)\right)$ approximates a Riemann sum for the integral of $S$ along $\gamma$ from $t=0$ to $t=2 K r$ and a uniform bound on the oscillation of the scalar curvature of the ambient manifold has been assumed. Furthermore, it is also the case that $\delta_{k}<\varepsilon^{1 / 2}$ for small $r$ simply by examining the dependence of the $E(r, \varepsilon, \delta)$ quantities on its arguments. This completes the construction of the finite-length CMC surface.

The one-ended surface. The equations that must be solved to produce the oneended CMC surface are slightly different. Using the same notation as above, these 
equations are

$$
\begin{aligned}
0 & =\delta_{1}+\varepsilon_{1}^{-3 / 2} E_{1,1}(r, \varepsilon, \delta) \\
& \vdots \\
0 & =\delta_{K-1}+\varepsilon_{K}^{-3 / 2} E_{1, K-1}(r, \varepsilon, \delta)
\end{aligned}
$$

as well as

$$
\begin{aligned}
0 & =q_{0}\left(\varepsilon_{0}\right)-C_{2,0} r^{3} \dot{S}\left(p_{0}\right)+E_{2,0}(r, \varepsilon, \delta) \\
0 & =-q_{0}\left(\varepsilon_{0}\right)+q_{1}\left(\varepsilon_{1}\right)-C_{2,1} r^{3} \dot{S}\left(p_{1}\right)+E_{2,1}(r, \varepsilon, \delta) \\
& \vdots \\
0 & =-q_{K-1}\left(\varepsilon_{k}\right)+q_{K}\left(\varepsilon_{K}\right)-C_{2, K} r^{3} \dot{S}\left(p_{K}\right)+E_{2, K}(r, \varepsilon, \delta) .
\end{aligned}
$$

One should again view the equations in (6.6) as a systems of equations for the $\varepsilon$ and $\delta$ variables to be treated using the implicit function theorem, but this time depending on the parameters $r$ and the point $p_{0}$. When $r=0$ and $p_{0}$ is any point on $\gamma$, there is an exact solution $\delta_{1}=\cdots=\delta_{K-1}=0$ and $\varepsilon_{1}=\cdots=\varepsilon_{K}=0$. Furthermore, the derivative matrix of the function of $(\varepsilon, \delta, r)$ defined by the right hand sides of (6.6) in the $(\varepsilon, \delta)$ variables is invertible at $r=0$ with a lower bound of size $\mathcal{O}(1)$ on its determinant. Hence by the inverse function theorem there is a solution of (6.5) for all sufficiently small $r$, the dependence of $\varepsilon$ and $\delta$ on $r$ is smooth, and the dependence of the solution on $r$ is the same as before. This completes the construction of the one-ended CMC surface.

\section{References}

[1] A. Butscher and F. PACARD, Doubling constant mean curvature tori in $S^{3}$, Ann. Sc. Norm. Super. Pisa Cl. Sci. (5) 5 (2006), 611-638.

[2] A. BUTSCHER and F. PACARD, Generalized doubling constructions for constant mean curvature hypersurfaces in $S^{n+1}$, Ann. Global Anal. Geom. 32 (2007), 103-123.

[3] K. Grosse-Brauckmann, N. KorevaAr, R. Kusner, J. Ratzkin and J. Sullivan, Coplanar k-unduloids are nondegenerate, Preprint: arXiv:0712.1865.

[4] K. Grosse-Brauckmann, R. Kusner and J. Sullivan, Triunduloids: embedded constant mean curvature surfaces with three ends and genus zero, J. Reine Angew. Math. 564 (2003), 35-61.

[5] K. Grosse-Brauckmann, R. Kusner and J. Sullivan, Coplanar constant mean curvature surfaces, Comm. Anal. Geom. 15 (2007), no. 5, 985-1023.

[6] N. Kapouleas, Complete constant mean curvature surfaces in Euclidean three-space, Ann. of Math. (2) 131 (1990), 239-330.

[7] N. Kapouleas, Compact constant mean curvature surfaces in Euclidean three-space, J. Differential Geom. 33 (1991), 683-715.

[8] N. KorevaAR, R. KUSNER and B. SOlOMOn, The structure of complete embedded surfaces with constant mean curvature, J. Differential Geom. 30 (1989), 465-503.

[9] R. Kusner, R. MAZzeo and D. Pollack, The moduli space of complete embedded constant mean curvature surfaces, Geom. Func. Anal. 6 (1996), 120-137. 
[10] R. MAZZEO and F. PACARD, Constant mean curvature surfaces with Delaunay ends, Comm. Anal. Geom. 9 (2001), 169-237.

[11] R. MAZZEO and F. PACARD, Foliations by constant mean curvature tubes, Comm. Anal. Geom. 13 (2005), 633-670.

[12] R. MAZZEO, F. PACARD and D. Pollack, Connected sums of constant mean curvature surfaces in Euclidean 3 space, J. Reine Angew. Math. 536 (2001), 115-165.

[13] R. MAZZEO, Recent advances in the global theory of constant mean curvature surfaces, In: "Noncompact Problems at the Intersection of Geometry, Analysis, and Topology", Contemp. Math., Vol. 350, Amer. Math. Soc., Providence, RI, 2004, 179-199.

[14] W. H. MEEKS, III, The topology and geometry of embedded surfaces of constant mean curvature, J. Differential Geom. 27 (1988), 539-552.

[15] F. PACARD, Connected Sum Constructions in Geometry and Nonlinear Analysis, preprint: http://perso-math.univ-mlv.fr/users/pacard.frank/Lecture-Part-I.pdf.

[16] F. PACARD, Surfaces à courbure moyenne constante, In: "Image des mathématiques 2006", Publications of the CNRS, CNRS, Paris, 2006, 107-112.

[17] J. RatzKIN, “An End-to-End Construction for Constant Mean Curvature Surfaces", Ph.D. thesis, University of Washington, 2001, preprint: http://www.math.uga.edu/ jratzkin/papers/thesis.pdf.

[18] H. ROSENBERG, Constant mean curvature surfaces in homogeneously regular 3-manifolds, Bull. Austral. Math. Soc. 74 (2006), 227-238.

[19] R. M. SCHOEN, The existence of weak solutions with prescribed singular behavior for a conformally invariant scalar equation, Comm. Pure Appl. Math. 41 (1988), 317-392.

[20] R. YE, Foliation by constant mean curvature spheres, Pacific J. Math. 147 (1991), 381-396.

Department of Mathematics Stanford University Stanford, CA 94305

butscher@math.stanford.edu mazzeo@math.stanford.edu 